\title{
AN AUTOMATED TEST STATION DESIGN USED TO VERIFY AIRCRAFT COMMUNICATION PROTOCOLS
}

\author{
A Thesis \\ presented to \\ the Faculty of California Polytechnic State University, \\ San Luis Obispo
}

\author{
In Partial Fulfillment \\ of the Requirements for the Degree \\ Master of Science in Aerospace Engineering
}

by

Joshua Christopher Berrian

October 2011 
(C) 2011

Joshua Christopher Berrian

ALL RIGHTS RESERVED 
TITLE:

AUTHOR:

DATE SUBMITTED:

COMMITTEE CHAIR:

COMMITTEE MEMBER: $\quad$ Kristina Jameson, Ph.D.

COMMITTE MEMBER: $\quad$ Eric Mehiel, Ph.D.
AN AUTOMATED TEST STATION DESIGN USED TO VERIFY AIRCRAFT COMMUNICATION PROTOCOLS

Joshua Christopher Berrian

October 2011 


\author{
ABSTRACT \\ AN AUTOMATED TEST STATION DESIGN USED TO VERIFY AIRCRAFT \\ COMMUNICATION PROTOCOLS \\ Joshua Christopher Berrian
}

\begin{abstract}
Requirements verification is typically the costliest part of the systems engineering design process. In the commercial aircraft industry, as the software and hardware design evolves, it must be verified to conform to requirements. In addition, when new design releases are made, regression analysis must be performed which usually requires repeat testing. To streamline verification, a suite of automated verification tools is described in this document which can reduce the test effort. This test suite can be qualified to be used to verify systems at any DO-178B design assurance level. Some of the software tools are briefly described below.

There are major advantages of this automated verification effort. The tools can either be internally developed by a company or purchased "off the shelf", depending upon budget and staff constraints. Every automated test case can be run with the click of a button and failures caused by human factors are reduced. The station can be qualified per DO-178B guidelines, and can also be expanded to support ARINC 429, AFDX, Ethernet, and MIL-STD-1553 interfaces. The expansion of these test programs would enable the creation of a universal avionics test suite with minimal cost and a reduction of the overall program verification effort.

The following is a presentation of an automated test station capable of reducing verification time and cost. The hardware and software aspects needed to create the test station are examined. Also, steps are provided to help guide a designer through the tool qualification process. Lastly, a full suite of test functions are included that can be implemented and customized to verify a wide range of avionics communication characteristics.
\end{abstract}




\section{TABLE OF CONTENTS}

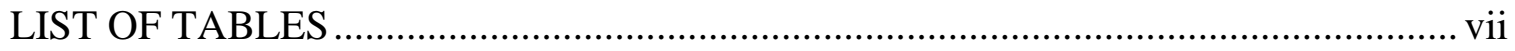

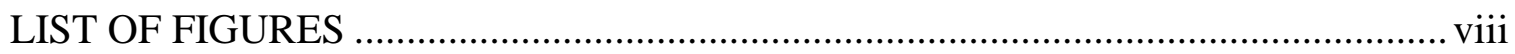

CHAPTER 1: AVIONICS COMMUNICATION OVERVIEW …………………............. 1

1.1 ARINC-429 Communication Protocol.......................................................................... 1

1.1.1 Avionics Usage .................................................................................. 1

1.1.2 Protocol Description ............................................................................. 1

1.1.3 Avionics Hardware Interface ................................................................... 2

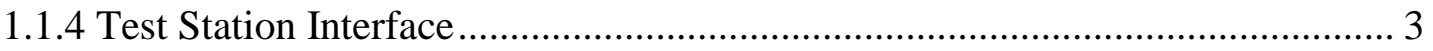

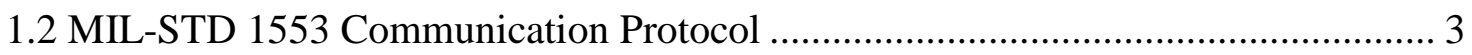

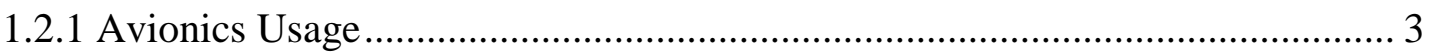

1.2.2 Protocol Description .............................................................................. 4

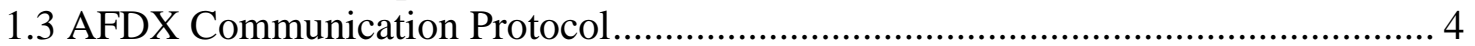

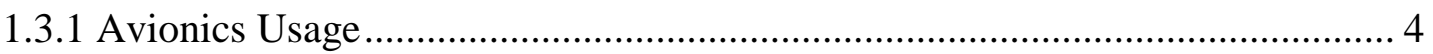

1.3.2 Avionics Hardware Interface ................................................................... 4

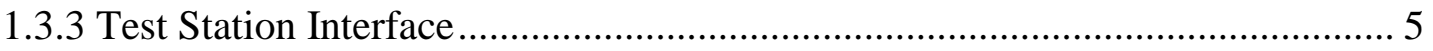

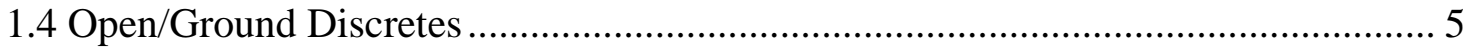

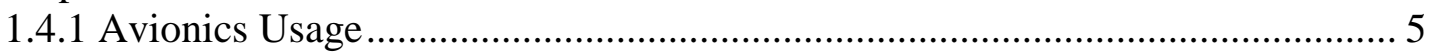

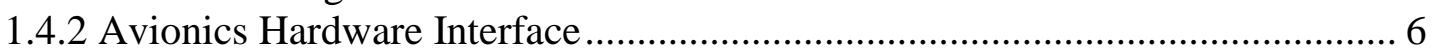

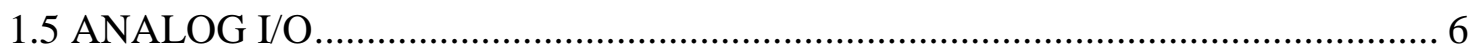

CHAPTER 2: AUTOMATED TEST STATION DESIGN.......................................... 7

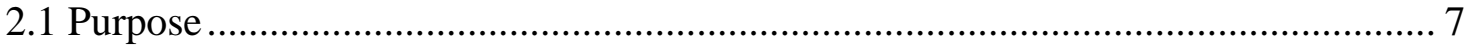

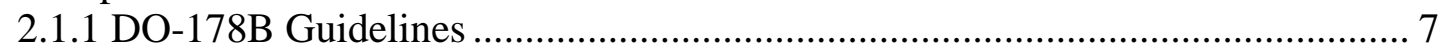

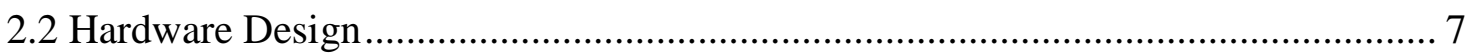

2.2.1 Test Station Hardware and Cost ................................................................. 8

2.2.1.1 National Instruments computer rack (PXI-1011) ...................................... 10

2.2.1.1.1 Input Discretes............................................................................... 11

2.2.1.1.2 Output Discretes and Analog Signals.................................................. 13

2.2.1.1.3 Bus interface hardware - ARINC 429 and AFDX............................. 14

2.2.1.1.4 Discrete Interface panel (power, control discrete, etc)........................ 16

2.2.2 Digitally Controlled DC Power Supplies .......................................................... 17

2.2.3 Motor Variac for AC Voltage Regulation ...................................................... 17

CHAPTER 3: SOFTWARE APPLICATIONS FOR TEST STATION USE ................... 18

3.1 Applications to Exercise Avionics Systems ............................................................ 18

3.1.1 ARINC Communication Organizer ........................................................... 18

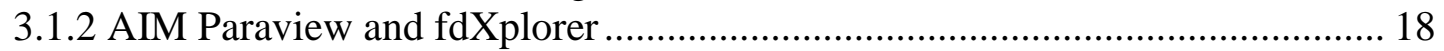

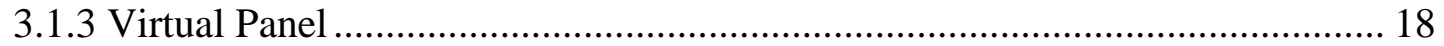

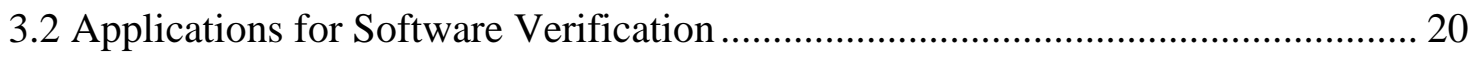

3.2.1 Mercury Interactive WinRunner....................................................................... 20 
3.2.1.1 Customized Function Library ........................................................ 20

3.2.1.1.1 Test Station Startup Functions ..................................................... 22

3.2.1.1.2 Verification Test User Functions .................................................. 22

3.2.1.1.3 ATP, EMI, and Environmental Test Functions................................ 23

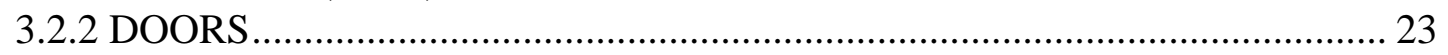

3.2.2.1 System Requirements Extraction and Synchronization ............................ 24

3.2.2.2 WinRunner Verification Test Shell Creation........................................... 24

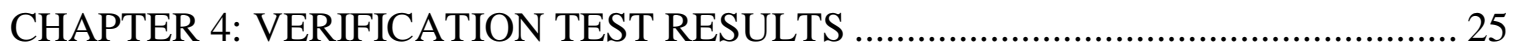

4.1 WinRunner Verification Data Generation........................................................... 25

4.2 WinRunner Verification Test Results ................................................................ 25

4.3 WinRunner Verification Test Execution............................................................. 28

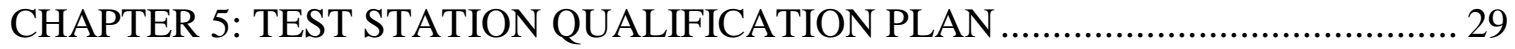

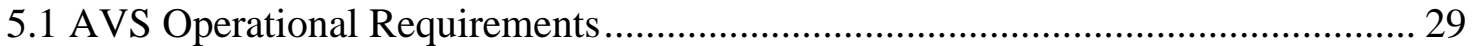

5.2 Function Library Compliance / Test .................................................................... 29

5.3 Test Coverage Compliance ............................................................................... 29

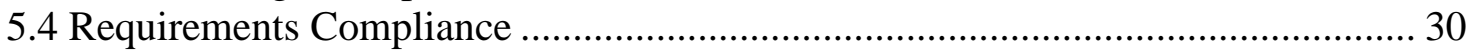

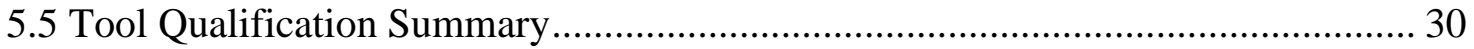

CHAPTER 6: TEST STATION SCOPE AND GENERAL USES ............................. 31

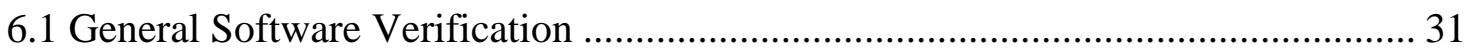

6.2 Hardware Acceptance and Environmental Testing ............................................. 31

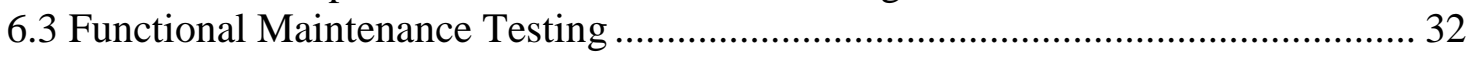

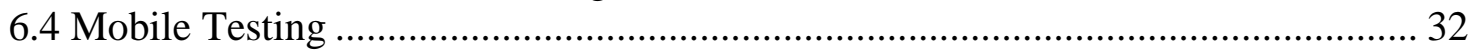

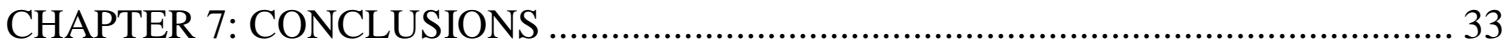

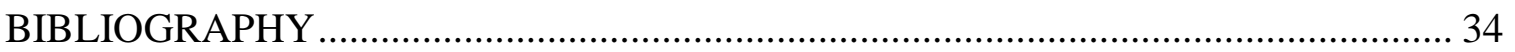

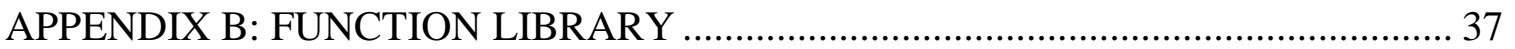




\section{LIST OF TABLES}

TABLE 1.1 ARINC-429 WORD STRUCTURE ..................................................... 1

TABLE 2.1 AUTOMATED TEST STATION HARDWARE COST …........................ 8 


\section{LIST OF FIGURES}

FIGURE 1.1 ARINC-600 CONNECTOR WITH 600 PINS PLUS POWER PINS ......... 3

FIGURE 1.2 AFDX CONNECTOR REPRESENTATION ....................................... 5

FIGURE 1.3 EXAMPLE OF AVIONICS SYSTEM OUTPUT DISCRETES ............... 5

FIGURE 2.1 BLOCK DIAGRAM OF TEST STATION HARDWARE....................... 10

FIGURE 2.2 SLOT 1 PCI-E CARD CONNECTED TO UUT INPUT DISCRETES .... 11

FIGURE 2.3 SLOT 2 PCI-E CARD CONNECTED TO UUT INPUT DISCRETES .... 12

FIGURE 2.4 SLOT 3 PCI-E CARD CONNECTED TO UUT ANALOG INPUT AND

DISCRETE OUTPUT 13

FIGURE 2.5 SLOT 4 PCI-E CARD CONNECTED TO UUT ARINC 429 INPUT

DATABUS 14

FIGURE 2.6 SLOT 5 PCI-E CARD CONNECTED TO UUT ARINC 429 OUTPUT

DATABUS 15

FIGURE 2.7 SLOT 6 PCI-E CARD CONNECTED TO UUT INPUT POWER 16

FIGURE 3.1 INPUT DISCRETE VIRTUAL PANEL .......................................... 19

FIGURE 3.2 OUTPUT DISCRETE VIRTUAL PANEL .......................................... 19

FIGURE 3.3 ANALOG CONTROLS VIRTUAL PANEL ......................................... 19

FIGURE 3.4 POWER CONTROLS VIRTUAL PANEL ........................................ 20

FIGURE 3.5 AUTOMATED TEST FLOW ….......................................................... 21

FIGURE 4.1 WINRUNNER VERIFICATION RESULTS IN A TEST SET............... 26

FIGURE 4.2 WINRUNNER VERIFICATION RESULTS FOR A SPECIFIC TEST ... 27 


\section{CHAPTER 1: AVIONICS COMMUNICATION OVERVIEW}

In order to facilitate information communication throughout an aircraft, several data transmission protocols can be employed. The following sections discuss the differences between the types of data signals.

\subsection{ARINC-429 Communication Protocol}

\subsubsection{Avionics Usage}

ARINC-429 is a data transmission protocol used throughout the commercial aircraft industry. An aircraft is comprised of many individual systems, ranging from fire detection to navigation. Commercial aircraft use ARINC-429 protocol as a means of communication between these systems in order to send system status, failure information, GPS, and other avionics data. For example, a navigation system can send heading, pitch, roll, etc. to the cockpit avionics displays using ARINC-429 data buses.

\subsubsection{Protocol Description}

The ARINC-429 protocol is defined as a 32-bit word that is structured as shown in Table 1.1. All ARINC-429 data is received in this format. The first 8 bits define the label number converted in octal. The label is organized with bit 7 as most signification bit (MSB), and bit 0 as least significant bit (LSB). The rest of the word is sent from LSB to MSB. Bit 8 is the ninth bit sent followed by bit 9 and so on until bit 31 . So, the transmission is organized first as bits 7-0, then 8-31 follow.

TABLE 1.1 ARINC-429 WORD STRUCTURE

\begin{tabular}{|c|c|c|c|c|c|}
\hline Bit Numbers. & 31 & $30-29$ & $28-10$ & 9,8 & $7-0$ \\
\hline Definition & Parity & SSM & Data (MSB-LSB) & SDI & Label (MSB-LSB) \\
\hline
\end{tabular}


The SSM stands for Sign/Status Matrix and is used to relay the validity of the data word. As the SSM is a 2-bit field, there are four states indicating validity: Normal Operation (NO), No Computed Data (NCD), Functional Test (FT), and Failure Warning (FW). A Normal Operation SSM indicates the data is valid with the origination system working as designed. An SSM of No Computed Data indicates the data may not be trustworthy due to a reason other than failure. For example, NCD could be indicated for inertial navigation data before the sensor package has fully aligned. Failure Warning is used for invalid data resulting from a failed software partition or entire system. Functional Test can be used when "canned" data is being streamed due to a known test setting.

\subsubsection{Avionics Hardware Interface}

Each ARINC data bus requires two wires (twisted pair) to facilitate data transmission between aircraft systems. Therefore, a main wiring harness may contain a bundle of hundreds of wires that are strung throughout the aircraft body. Specialized ARINC backplate connectors such as the ARINC-600 connector allow for organization of data bus connections with many pins. 


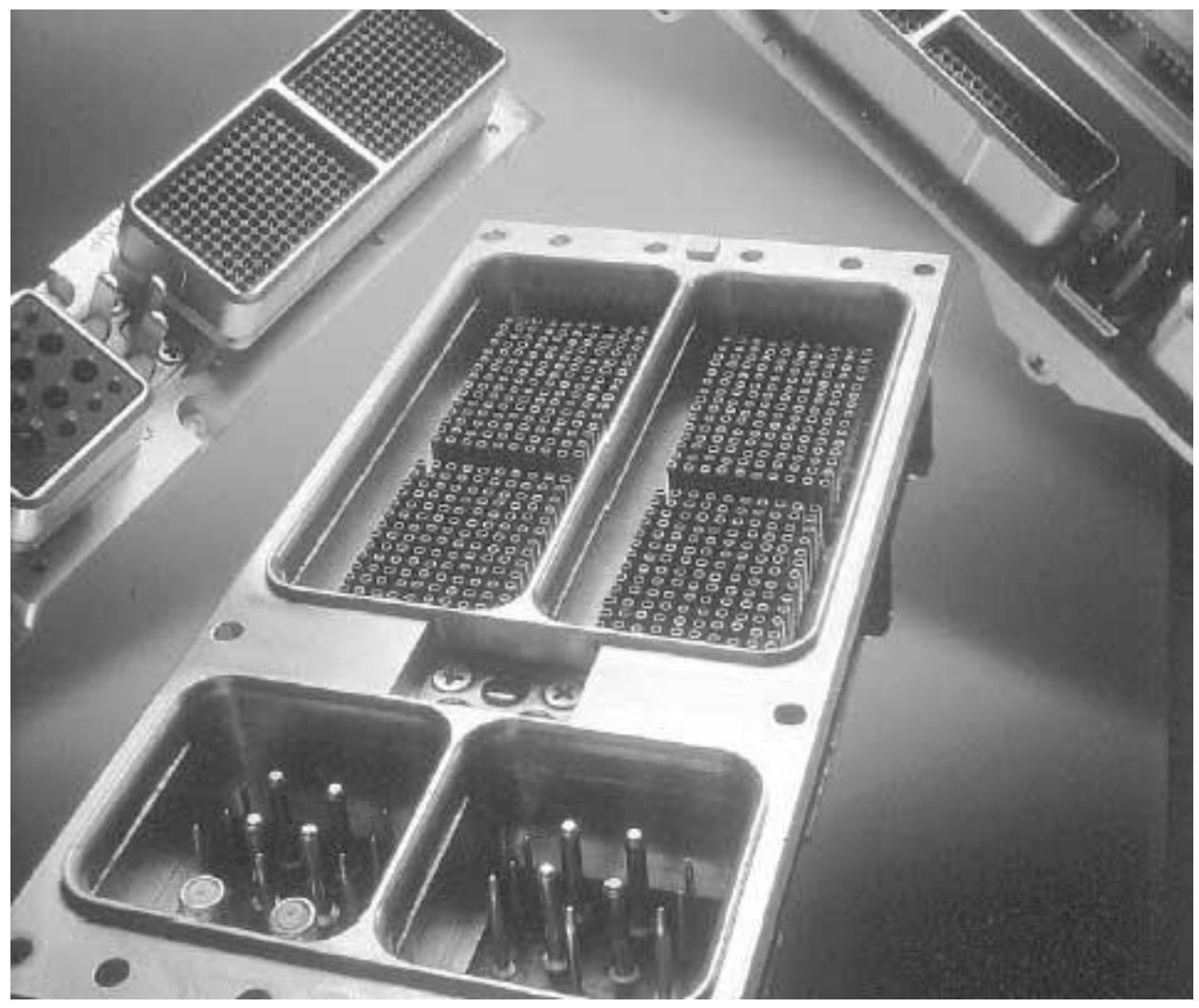

FIGURE 1.1 ARINC-600 CONNECTOR WITH 600 PINS PLUS POWER PINS

\subsubsection{Test Station Interface}

The test station can be upgraded to accommodate all ARINC type connections. The wiring harness would be fitted to match that which would mount in the aircraft.

\subsection{MIL-STD 1553 Communication Protocol}

\subsubsection{Avionics Usage}

MIL-STD-1553 was developed to alleviate the growing wire harness weight of increasingly complex aircraft systems. This protocol offered a common data bus that could facilitate communication using a destination identifier. The receiving system can disseminate data that was intended for its destination terminal. MIL-STD-1553 is widely used in the military aviation field. Each 
system on the aircraft can be configured to be a remote terminal, which can be reached by the bus controller.

\subsubsection{Protocol Description}

This standard uses words that are 20 bits long. 1 bit is used for parity, 3 bits are used for sync and the other 16 bits hold the data payload. Like Ethernet and AFDX protocols, 1553 messages are sent in packets or groups of up to 32 data words from the bus controller to a remote terminal.

\subsection{AFDX Communication Protocol}

\subsubsection{Avionics Usage}

Avionics Full Duplex Switched Ethernet (AFDX) was developed by Airbus Industries to provide an integrated high speed Ethernet network in aircraft. This network is defined by communication from end-systems to and from switches. Each line replaceable unit (LRU) on the aircraft could be defined as an end-system. These endsystems use IP addresses and MAC destination fields to route their data.

\subsubsection{Avionics Hardware Interface}

The figure shown below is a representation of an AFDX network connection which includes inherent redundancy. Transmissions are sent simultaneously across the $\mathrm{TX}+$ and TX- wires while data is received across the RX+ and RX- wires. These channels are wired in a tisted pair configuration. 


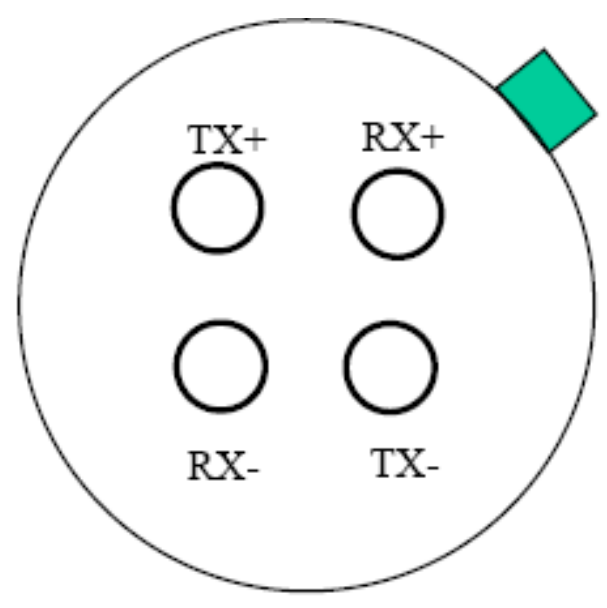

\section{FIGURE 1.2 AFDX CONNECTOR REPRESENTATION}

\subsubsection{Test Station Interface}

The ARINC 600 connector provides capability for AFDX connections. The test station wiring harness can be configured to support numerous AFDX networks.

\subsection{Open/Ground Discretes}

Input discretes on each $\mathrm{I} / \mathrm{O}$ can be set to either the OPEN condition or to the ground (GND) condition. In the OPEN condition the corresponding register bit would read "1", and in GND would read "0".

\subsubsection{Avionics Usage}

Input discretes are generally used to set initial conditions upon system startup.

They can be set to include mode control (normal operation, safe mode, maintenance, etc), aircraft type (Airbus, Boeing, etc.), power on/off, and various other avionics options. Output discretes are used to indicate states, settings, or modes of a system.

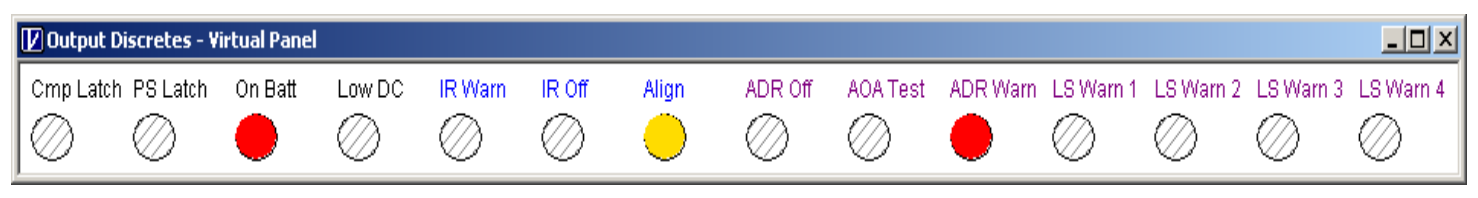

FIGURE 1.3 EXAMPLE OF AVIONICS SYSTEM OUTPUT DISCRETES 


\subsubsection{Avionics Hardware Interface}

Each input and output discrete will be assigned to their own pin in the system connector. These can then be wired to the test station control panel

\subsection{ANALOG I/O}

Analog signals are used frequently in aircraft design. They are widely used to relay air data information. Examples include: Angle-of-Attack (AOA) resolver inputs, Barometric pressure resolver inputs, voltage reference inputs, and Total Air Temperature (TAT) inputs. 


\section{CHAPTER 2: AUTOMATED TEST STATION DESIGN}

\subsection{Purpose}

The purpose of this project is to design a universal, system level, Automated Verification Station (AVS) for that can interface with general avionics data communication interfaces. System level testing refers to verification at the "black box" level. This level of testing includes simulating inputs and reading the resulting outputs of a unit under test (UUT). However, with the use of a memory inspector utility (MIU), data can be input or read directly from system memory. The basic design of this test station is to provide inputs to and measure outputs from the UUT.

\subsubsection{DO-178B Guidelines}

Software Considerations in Airborne Systems and Equipment Certification, or DO-178B, was published by RTCA, Incorporated. The Federal Aviation Administration (FAA) uses DO-178B as a guideline to verify that software for an airborne system will perform reliably. It is not part of the scope of this project to detail DO-178B usage, but to merely relay the possible constraits that it may place on an aircraft system in development.

\subsection{Hardware Design}

For an automated test station design, various computing packages can be used. National Instruments provides several options that can allow for test station growth and adaptation to different environments. The availability of future growth options is very important when designing for aircraft systems. These systems need to be maintainable and upgradeable as aircraft systems can be in usage for decades. As components become obsolete or end production, replacements can be very expensive or impossible to find,

and testing can be a difficult process. Also, when dealing with DO-178B guidelines, it 
may neccessary to qualify every update to test station hardware and software. Clearly, it would be beneficial to only have to verify the test station once during a program lifespan.

\subsubsection{Test Station Hardware and Cost}

TABLE 2.1 AUTOMATED TEST STATION HARDWARE COST

\begin{tabular}{|c|c|c|c|c|}
\hline Company & Description & Qty & Price & Total \\
\hline Techmar Enclosures & Interface Chassis & 1 & $\$ 240.00$ & $\$ 240.00$ \\
\hline Staco Energy Products & Motorized Variable Transformer & 1 & $\$ 669.00$ & $\$ 669.00$ \\
\hline Pacific Design & Machine Front Panel Interface & 1 & $\$ 285.00$ & $\$ 285.00$ \\
\hline Pacific Design & Machine Rear Panel Interface & 1 & $\$ 365.00$ & $\$ 365.00$ \\
\hline National Instruments & SCXI-1310 96 Pin Connector & 6 & $\$ 50.00$ & $\$ 300.00$ \\
\hline National Instruments & Cable, HV8-BAN4 & 1 & $\$ 40.00$ & $\$ 40.00$ \\
\hline National Instruments & PXI-1011 Rack & 1 & $\$ 2,795.00$ & $\$ 2,795.00$ \\
\hline National Instruments & PCI to PXI Kit & 1 & $\$ 1,495.00$ & $\$ 1,495.00$ \\
\hline National Instruments & SCXI-1181 Breadboard Module & 1 & $\$ 195.00$ & $\$ 195.00$ \\
\hline National Instruments & SCXI-1160 16Ch Relay & 5 & $\$ 895.00$ & $\$ 4,475.00$ \\
\hline National Instruments & SCXI-1127 HV Multiplexer & 1 & $\$ 995.00$ & $\$ 995.00$ \\
\hline National Instruments & SCXI-1331 64 ch. Termin. Blk. & 1 & $\$ 180.00$ & $\$ 180.00$ \\
\hline National Instruments & NI-4070 PXI Voltmeter & 1 & $\$ 1,995.00$ & $\$ 1,995.00$ \\
\hline North Atlantic Instruments & 4-Dig to Resolver CPCI Card & 1 & $\$ 4,735.00$ & $\$ 4,735.00$ \\
\hline Mercury Interactive & DC Power Supplies & 2 & $\$ 964.41$ & $\$ 1,928.82$ \\
\hline Mercury Interactive & Flange Kit & 1 & $\$ 53.22$ & $\$ 53.22$ \\
\hline Mercury Interactive & Lock Link Kit & 1 & $\$ 70.78$ & $\$ 70.78$ \\
\hline Amco Engineering Co. & Console -Blue & 1 & $\$ 421.00$ & $\$ 421.00$ \\
\hline Amco Engineering Co. & Castors & 4 & $\$ 18.00$ & $\$ 72.00$ \\
\hline Amco Engineering Co. & Panel 8.75" & 2 & $\$ 20.00$ & $\$ 40.00$ \\
\hline Amco Engineering Co. & Chassis Guide & 6 & $\$ 20.00$ & $\$ 120.00$ \\
\hline Condor Engineering & 16 Channel ARINC PCI Board & 1 & $\$ 6,000.00$ & $\$ 6,000.00$ \\
\hline Condor Engineering & 8 Channel ARINC PCI Board & 1 & $\$ 4,268.00$ & $\$ 4,268.00$ \\
\hline AIM & 2 Ch. AFDX CPCI Interface & 0 & $\$ 9,844.00$ & $\$ 9,844.00$ \\
\hline AIM & AFDX Analyzer Software & 0 & $\$ 5,995.00$ & $\$ 5,995.00$ \\
\hline AIM & AFDX Traffic Generator & 0 & $\$ 3,995.00$ & $\$ 3,995.00$ \\
\hline TECH SAT & $\begin{array}{l}\text { Intelligent ARINC } 429 \text { PCI- } \\
\text { Interface }\end{array}$ & 1 & $\$ 2,840.50$ & $\$ 2,840.50$ \\
\hline TECH SAT & $\begin{array}{l}\text { A330/340 CMC Simulation } \\
\text { Software }\end{array}$ & 1 & $\$ 2,840.50$ & $\$ 2,840.50$ \\
\hline Computer Cable Makers & 68 Pin SCSI F to F & 2 & $\$ 32.00$ & $\$ 64.00$ \\
\hline Newark Electronics & 68 Pin SCSI M to M 6' & 2 & $\$ 30.00$ & $\$ 60.00$ \\
\hline Newark Electronics & DB-37 Cable M to F 6' & 4 & $\$ 17.61$ & $\$ 70.44$ \\
\hline Newark Electronics & DB-9 Cable F to F 6' & 2 & $\$ 4.72$ & $\$ 9.44$ \\
\hline Newark Electronics & DB-37 Male Connector & 3 & $\$ 10.76$ & $\$ 32.28$ \\
\hline Newark Electronics & DB-25 Male Connector & 7 & $\$ 3.60$ & $\$ 25.20$ \\
\hline Newark Electronics & DB-25 Female Connector & 7 & $\$ 4.63$ & $\$ 32.41$ \\
\hline Newark Electronics & DB-9 Female Connector & 2 & $\$ 2.57$ & $\$ 5.14$ \\
\hline Newark Electronics & DB-9 Male Connector & 2 & $\$ 1.85$ & $\$ 3.70$ \\
\hline Newark Electronics & RJ-45 Coupler (AFDX) & 2 & $\$ 17.07$ & $\$ 34.14$ \\
\hline Newark Electronics & DB-25 Backshell Kit & 7 & $\$ 1.49$ & $\$ 10.43$ \\
\hline Newark Electronics & DB-9 Backshell Kit & 2 & $\$ 1.50$ & $\$ 3.00$ \\
\hline Newark Electronics & 8PC4627 AC Panel mount Plug & 1 & $\$ 0.68$ & $\$ 0.68$ \\
\hline
\end{tabular}




\begin{tabular}{|l|l|r|l|l|}
\hline Newark Electronics & 2086 AC Aux Plug & 1 & $\$ 2.54$ & $\$ 2.54$ \\
\hline Newark Electronics & 5 Pin Box Mount Receptacle & 1 & $\$ 17.36$ & $\$ 17.36$ \\
\hline Newark Electronics & 5 Pin Box Mount Receptacle & 1 & $\$ 14.40$ & $\$ 14.40$ \\
\hline Newark Electronics & 5 Pin Straight Plugs & 1 & $\$ 30.77$ & $\$ 30.77$ \\
\hline Newark Electronics & 5 Pin Straight Plugs & 1 & $\$ 33.23$ & $\$ 33.23$ \\
\hline Newark Electronics & Toggle Switch (Large) & 2 & $\$ 13.35$ & $\$ 26.70$ \\
\hline Newark Electronics & 115V 400Hz Flanged Inlet & 1 & $\$ 13.92$ & $\$ 13.92$ \\
\hline Newark Electronics & 115V 400Hz Plug & 1 & $\$ 16.12$ & $\$ 16.12$ \\
\hline Quality Time Computers & 2+GHz Computer & 1 & $\$ 619.00$ & $\$ 619.00$ \\
\hline Quality Time Computers & 18" Active Matrix Monitor & 2 & $\$ 590.00$ & $\$ 1,180.00$ \\
\hline & & & & \\
\hline & $\begin{array}{l}\text { TOTAL COST PER } \\
\text { STATION }\end{array}$ & & & $\mathbf{\$ 5 9 , 5 5 7 . 7 2}$ \\
\hline
\end{tabular}

Figure 2.1 shows a block diagram of the test station hardware connections.

Details of the hardware and how it is controlled are given in the following sub-sections. 


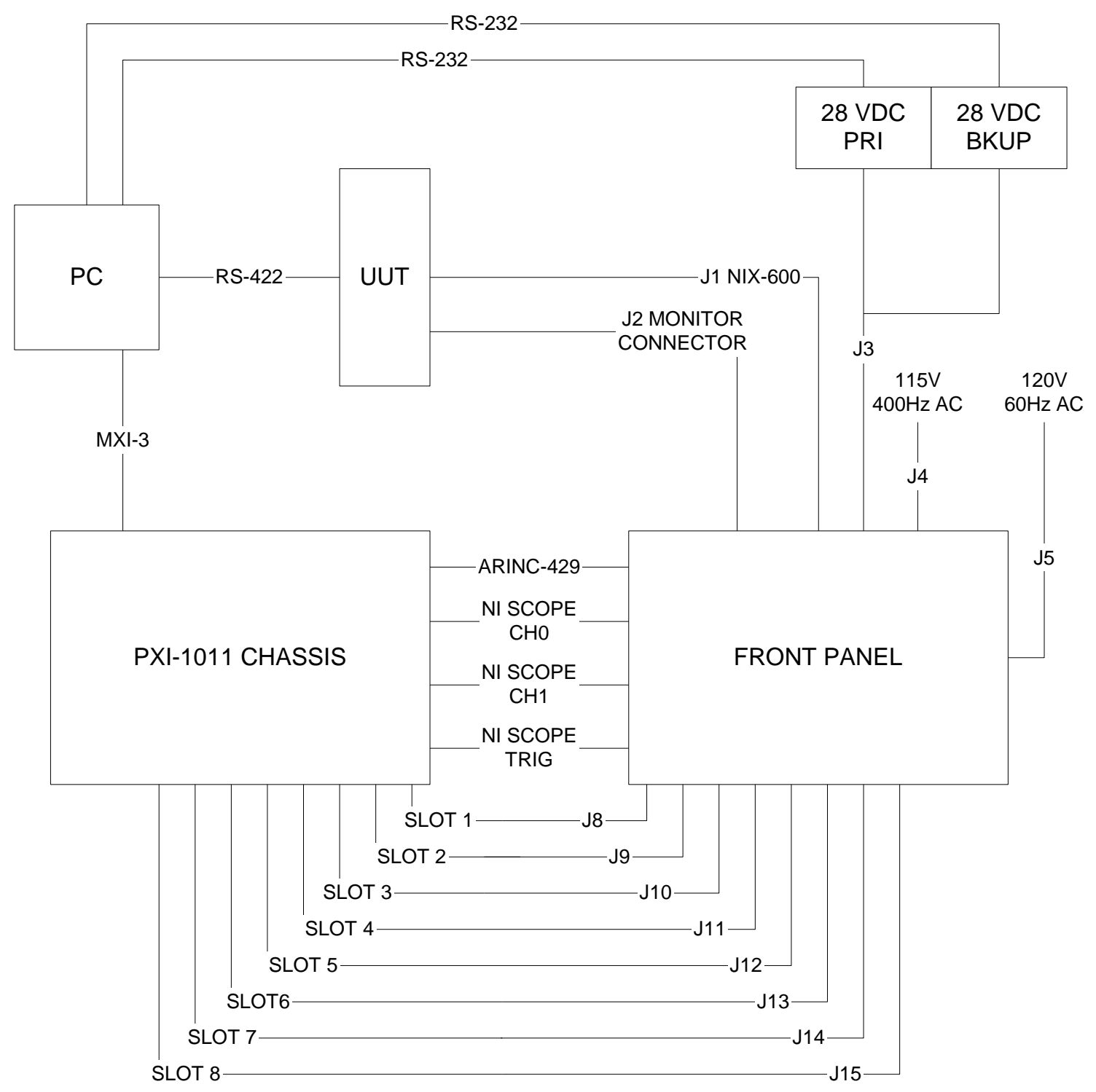

FIGURE 2.1 BLOCK DIAGRAM OF TEST STATION HARDWARE

\subsubsection{National Instruments computer rack (PXI-1011)}

The PXI-1011 provides PCI-E slots that facilitate the connections between the UUT and test computer. The slot configurations are described in the following sections. 


\subsection{Input Discretes}

\begin{tabular}{|c|c|c|c|c|c|}
\hline \multirow{5}{*}{ TB3 } & SCXI-1163R & J9 & NIX-600 & $J 1$ & \multirow[b]{2}{*}{ AUTחDADS SFI } \\
\hline & 0 & $A 1$ & & MP-J12 & \\
\hline & 1 & $\mathrm{~A} 2$ & & MP-J13 & ADR/DADS SEL \\
\hline & 2 & A3 & & $M P-A 2$ & MLUNT PISN 1 \\
\hline & 3 & A4 & & $M P-A 3$ & MLUNT PUSN 2 \\
\hline \multirow[t]{5}{*}{ E2 } & & A5 & OCDM & $T P-G 5$ & PRDG PIN CIM \\
\hline & 4 & $A 6$ & & MP-03 & CDU PRES \\
\hline & 5 & A7 & & MP-D11 & IR $\square F F$ \\
\hline & 6 & A8 & & $M P-K 15$ & AIR/GND \\
\hline & 7 & $\mathrm{C} 1$ & & MP-E9 & IR I/F TEST \\
\hline \multirow[t]{5}{*}{$\mathrm{E} 2$} & & C2 & $1 \mathrm{CDM}$ & & \\
\hline & 8 & C3 & - & & \\
\hline & 9 & C4 & & $\mathrm{TP}-\mathrm{C} 1$ & CAL WR ENBL \\
\hline & 10 & C5 & & $T P-C 7$ & PGM WR ENBL \\
\hline & 11 & C6 & & $\mathrm{TP}-\mathrm{B} 1$ & FAIL INHIBIT \\
\hline \multirow{5}{*}{ E2 } & & C7 & टC०M & & \\
\hline & 12 & c8 & & $M P-F 1$ & M1 MUDE SEL \\
\hline & 13 & E1 & & MP-F2 & M己 MDDE SLE \\
\hline & 14 & E2 & & MP-E7 & IR/ADIRS SEL \\
\hline & 15 & E3 & - & & \\
\hline \multirow{5}{*}{ E2 } & & E4 & उC口M & & \\
\hline & 16 & E5 & & $\mid T P-J 2$ & $A \square A \quad C D R R \quad 1 A$ \\
\hline & 17 & E6 & & TP-F12 & $A \square A \quad C D R R \quad 1 B$ \\
\hline & 18 & E7 & & TP-F 11 & $A \square A \quad C D R R \quad 2 A$ \\
\hline & 19 & E8 & & $T P-F 14$ & $A D A \quad C D R R \quad 2 B$ \\
\hline \multirow{5}{*}{ Е2 } & & K1 & 4C口M & & \\
\hline & 20 & $\mathrm{k} 2$ & & $\mathrm{TP}-\mathrm{H} 2$ & SSEC SEL 1 \\
\hline & 21 & $\mathrm{k} 3$ & & $\mathrm{TP}-\mathrm{K} 2$ & SSEL SEL $?$ \\
\hline & 22 & K4 & - & & \\
\hline & 23 & K5 & & MP-J5 & SPARE IDSC 7 \\
\hline \multirow[t]{5}{*}{ E2 } & & K6 & $5 C \square M$ & & \\
\hline & 24 & K7 & & $T P-K 4$ & ICE DET HEAT \\
\hline & 25 & K8 & & $T P-J 4$ & PITOT HEAT \\
\hline & 26 & M1 & & $\mathrm{TP}-\mathrm{H} 4$ & R STAT HEAT \\
\hline & 27 & M2 & & $\mathrm{TP}-\mathrm{G} 4$ & L STAT HEAT \\
\hline \multirow[t]{5}{*}{ E2 } & & M3 & 6C口M & & \\
\hline & 28 & M4 & & \multirow{5}{*}{$\begin{array}{l}\mathrm{TP}-\mathrm{F} 4 \\
\mathrm{TP}-\mathrm{J} 3 \\
\mathrm{TP}-\mathrm{H} 3 \\
\mathrm{MP}-\mathrm{K} 3\end{array}$} & TAT HEAT \\
\hline & 29 & M5 & & & $A \square A 1$ HEAT \\
\hline & 30 & M6 & & & $A \square A ~ 2$ HEAT \\
\hline & 31 & M7 & & & SPARE IDSC 8 \\
\hline E2 & & M8 & $7 C \square M$ & & \\
\hline
\end{tabular}

FIGURE 2.2 SLOT 1 PCI-E CARD CONNECTED TO UUT INPUT DISCRETES 


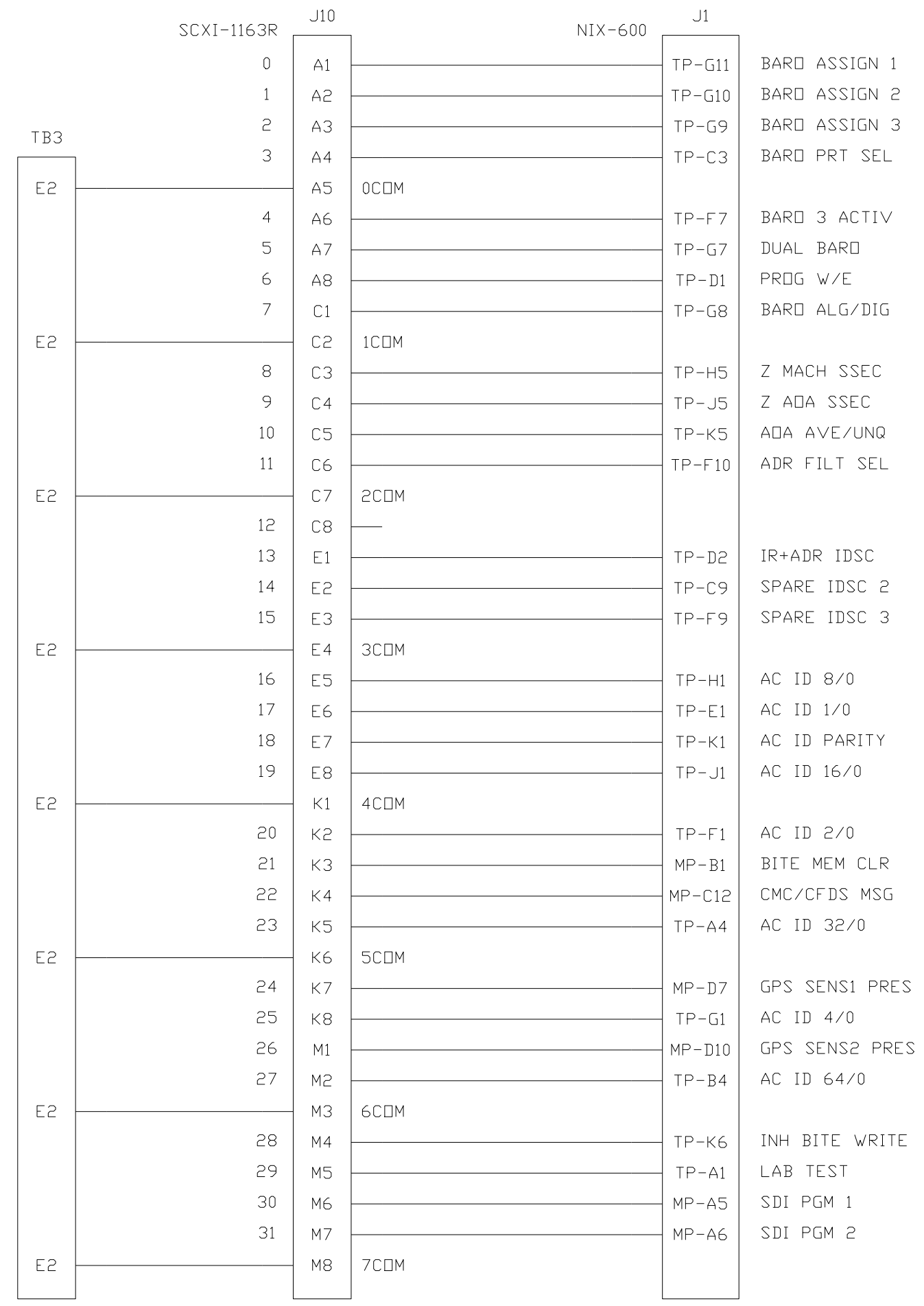

FIGURE 2.3 SLOT 2 PCI-E CARD CONNECTED TO UUT INPUT DISCRETES 


\subsection{Output Discretes and Analog Signals}

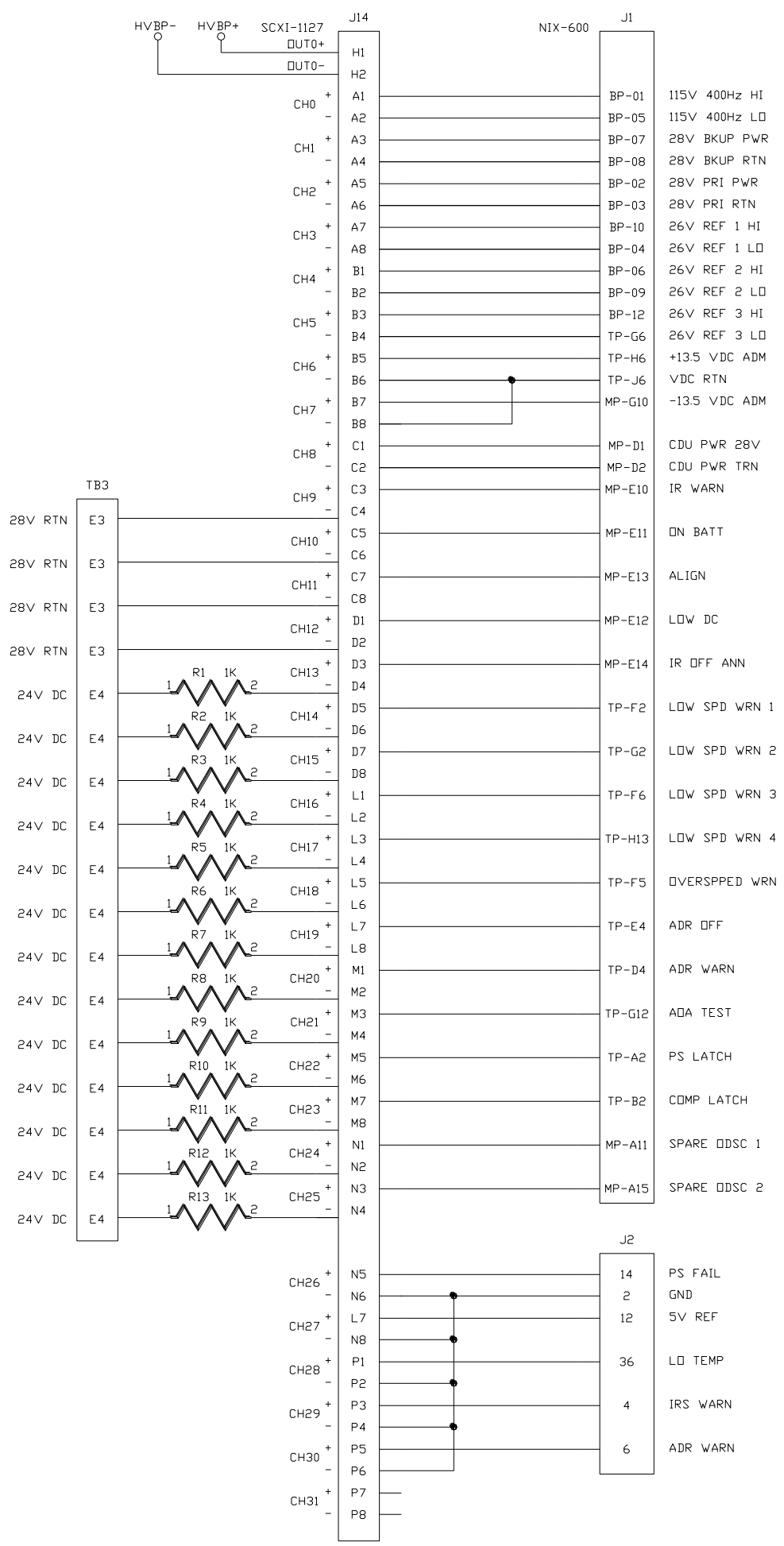

FIGURE 2.4 SLOT 3 PCI-E CARD CONNECTED TO UUT ANALOG INPUT AND DISCRETE OUTPUT 


\subsection{Bus interface hardware - ARINC 429 and AFDX}
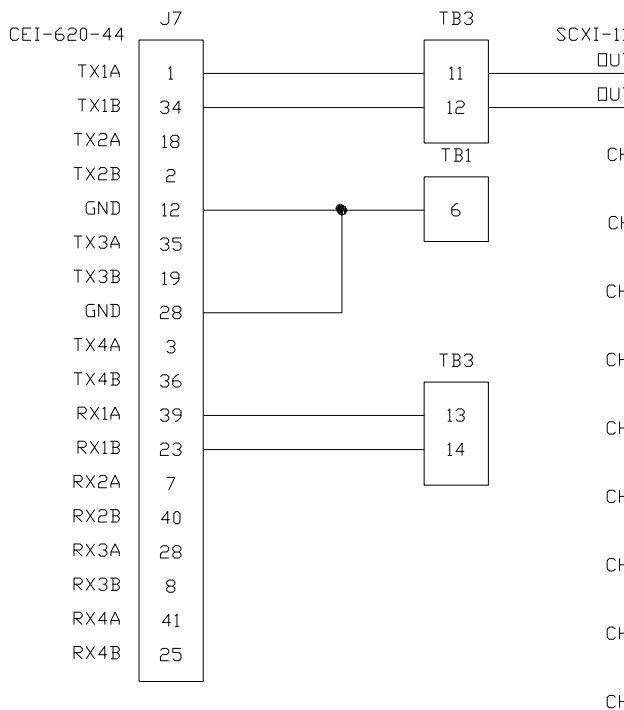

$\begin{array}{ll} & \\ & \\ 12\end{array}$

QUTO-

$\mathrm{CHO}^{+}$

$\mathrm{CH}^{+}$

$\mathrm{CH}^{+}$

$+\quad A 5$

$\mathrm{CH}^{+}$

$+\quad A 7$

$+\quad B$

$\mathrm{CH} 4$

$\mathrm{CH} 5$

$\mathrm{CH}^{+}$

$\mathrm{CH} 7^{+}$

$\mathrm{CH}^{+}$

$\mathrm{Cl}$

$\mathrm{CHO}^{+} \mathrm{C3}$

- $\mathrm{C}$

CHIO

C5
$-\quad 66$

$\mathrm{Cb}$
$+\quad 7$

- $\mathrm{C8}$

$\mathrm{CH}_{12}$

$$
\mathrm{CH}^{+}{ }_{-}^{+}
$$

D1

步

$\mathrm{CH}_{14}{ }^{+}$D5

$\mathrm{CH}_{15}{ }^{+}$

$\mathrm{CH}^{+}{ }^{+}$

$\mathrm{CH}^{+}{ }^{+}$

$$
\mathrm{CH}^{\mathrm{CH} 18}{ }^{+}
$$

$\mathrm{CH}_{-}^{+}$

$\mathrm{CH}_{19}^{+}$
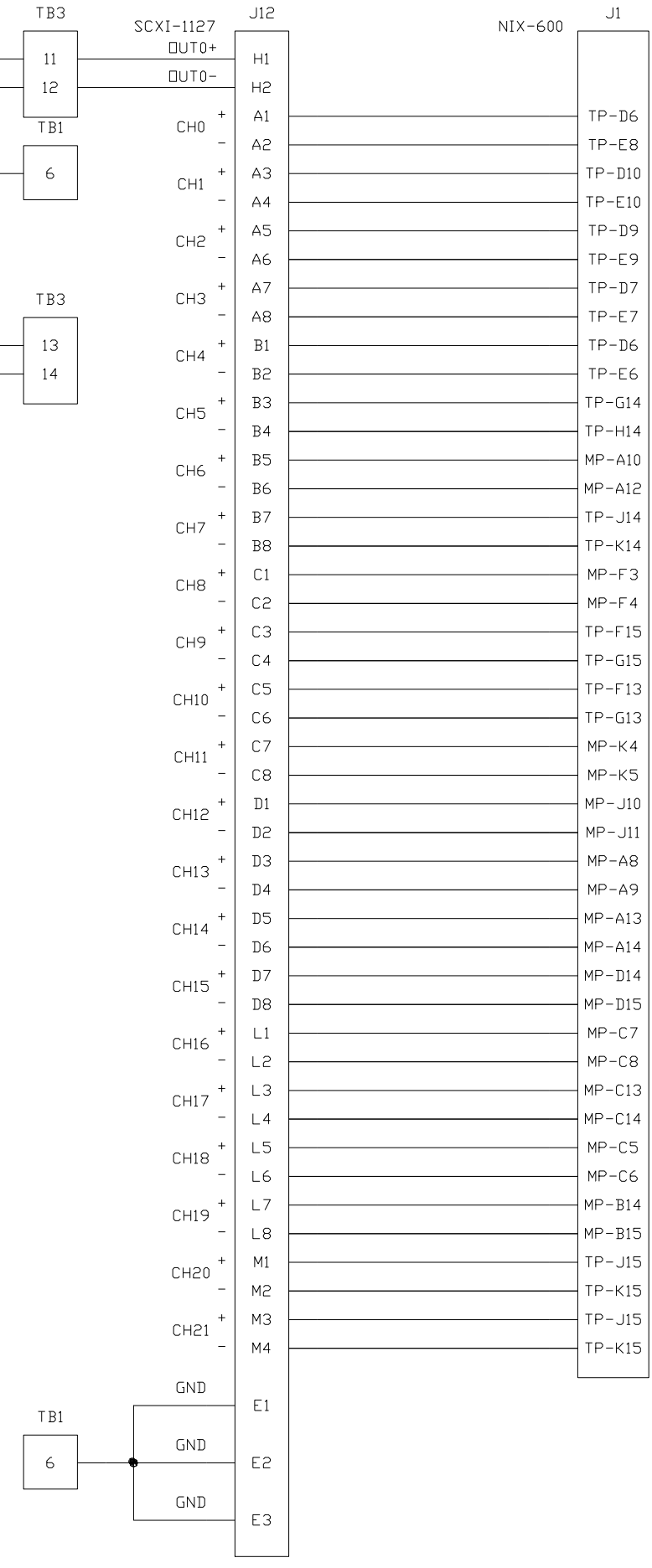

ADM IDB $3 A$ ADM IDB 3B ADM IDB $1 A$ ADM IDB $1 B$ $A D M$ IDB $2 A$ $A D M$ IDB $2 B$ $A D M$ IDB $4 A$ ADM IDB $4 B$ ADM IDB 5A ADM IDB 5B SPARE IDB IA SPARE IDB 1B RESVD GPS $1 A$ RESVD GPS $1 \mathrm{~B}$ SPARE IDB $2 A$ SPARE IDB $2 B$ RESVD GPS $2 A$ RESVD GPS $2 B$ BARQ IDB $2 A$ BARL IDB $2 B$ BARL IDB $1 A$ BARL IDB $2 A$ DADS IDB $1 A$ DADS IDB $1 \mathrm{~B}$ DADS IDB $2 A$ DADS IDB $2 B$ FMS IDB $1 A$ FMS IDB 1B FMS IDB $2 A$ FMS IDB $2 B$ GPSPRI IDB A GPSPRI IDB B GPSSEC IDB A GPSSEC IDB B CMC IDB A CMC IDB B CDU IDB A CDU IDB B DDU IDB A DDU IDB B ICM IDB $1 \mathrm{~A}$ ICM IDB $1 B$ ICM IDB 2A ICM IDB $2 B$

FIGURE 2.5 SLOT 4 PCI-E CARD CONNECTED TO UUT ARINC 429 INPUT DATABUS 


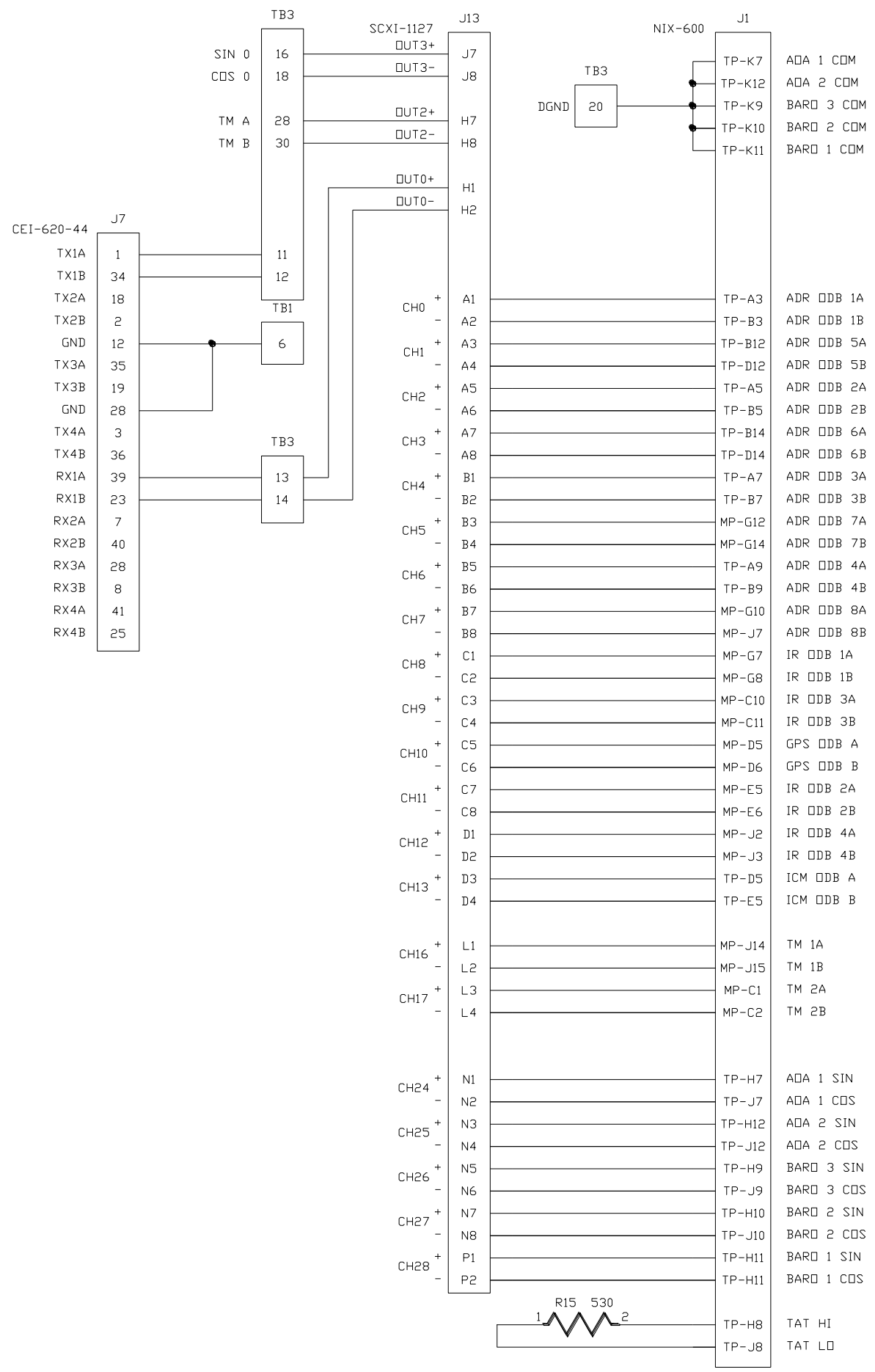

FIGURE 2.6 SLOT 5 PCI-E CARD CONNECTED TO UUT ARINC 429 OUTPUT DATABUS 
2.2.1.1.4 Discrete Interface panel (power, control discrete, etc)

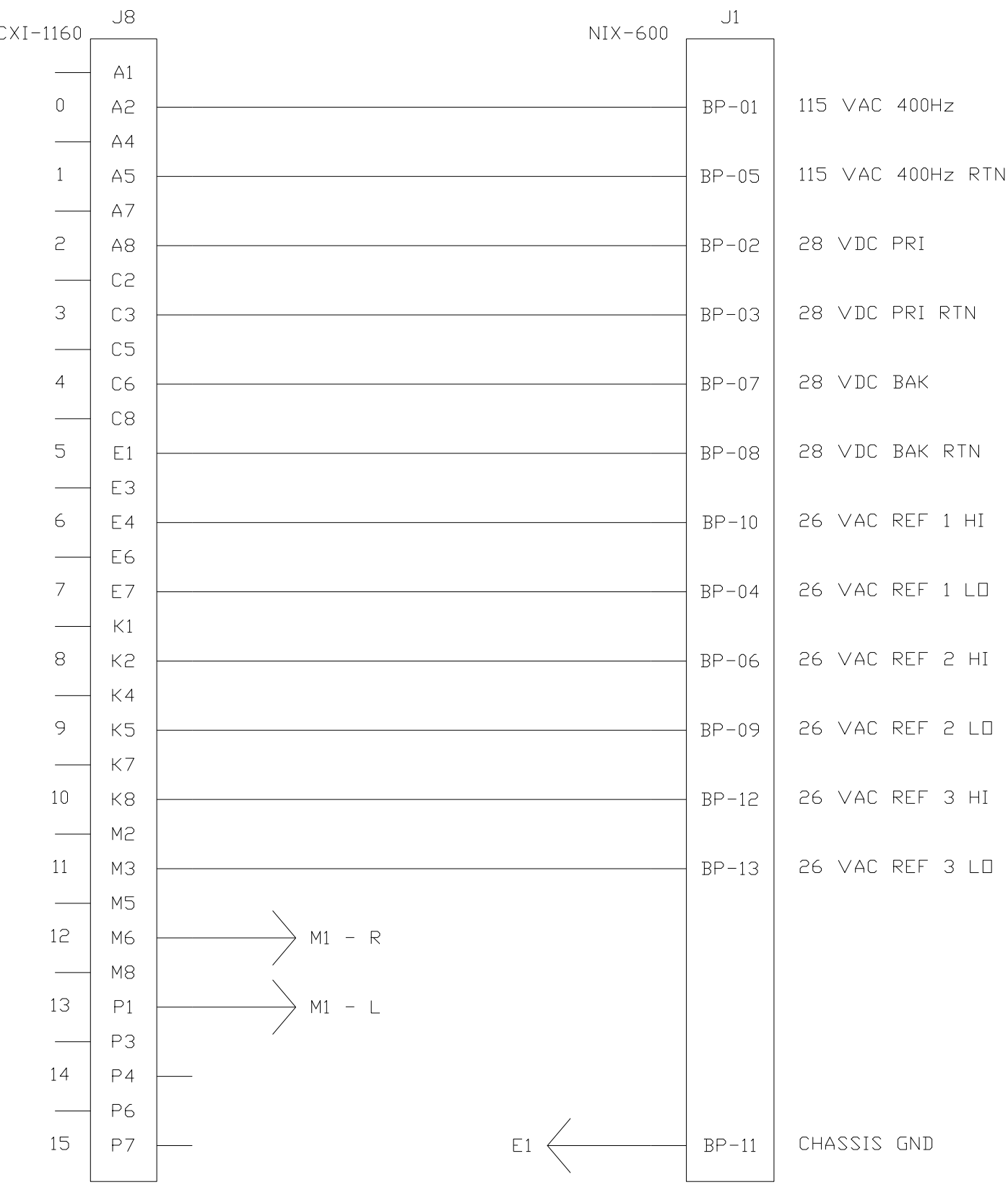

FIGURE 2.7 SLOT 6 PCI-E CARD CONNECTED TO UUT INPUT POWER 


\subsubsection{Digitally Controlled DC Power Supplies}

Two Mercury Interactive DC power supplies are used to provide primary and backup battery power to the system. Controls from the test computer can set the input voltages to the desired level.

\subsubsection{Motor Variac for AC Voltage Regulation}

A motor variac is used to adjust a primary input voltage with alternating current. When the variac motor is rotated, one can adjust the voltage up or down. This control is handled by the Power Control Virtual Panel resident on the test computer, which is described in following sections. 


\section{CHAPTER 3: SOFTWARE APPLICATIONS FOR TEST STATION USE}

\subsection{Applications to Exercise Avionics Systems}

\subsubsection{ARINC Communication Organizer}

ACO is a software application that simulates and transmits ARINC 429 inputs (labels) to and receives output channels from the UUT. Other programs are available offthe-shelf. For example, Mystic is available from Excaliber Systems.

\subsubsection{AIM Paraview and fdXplorer}

In usage, fdXplorer is analogous to the ACO except it is used for AFDX buses instead of ARINC 429. An AFDX input setup can be loaded into fdXplorer and it can capture the resulting output from the UUT. Paraview is a user-friendly GUI shell that organizes the ADFX packets into data words. Both fdXplorer and Paraview are available from AIM GmbH.

Wireshark (from the Wireshark Foundation) can also be used to record outputs from the UUT. It can "sniff" the data pertaining to specific IP addresses. However, Wireshark may only be used for data reception, not transmission.

\subsubsection{Virtual Panel}

The Virtual Panel is a representation of the direct connections between the test computer and the UUT. The routing is handled and controlled through the PXI-1011 National Instruments rack. Al signals are controlled by the Virtual Panel except for the ARINC 429 and AFDX data buses. The panels are shown in the following figures. 


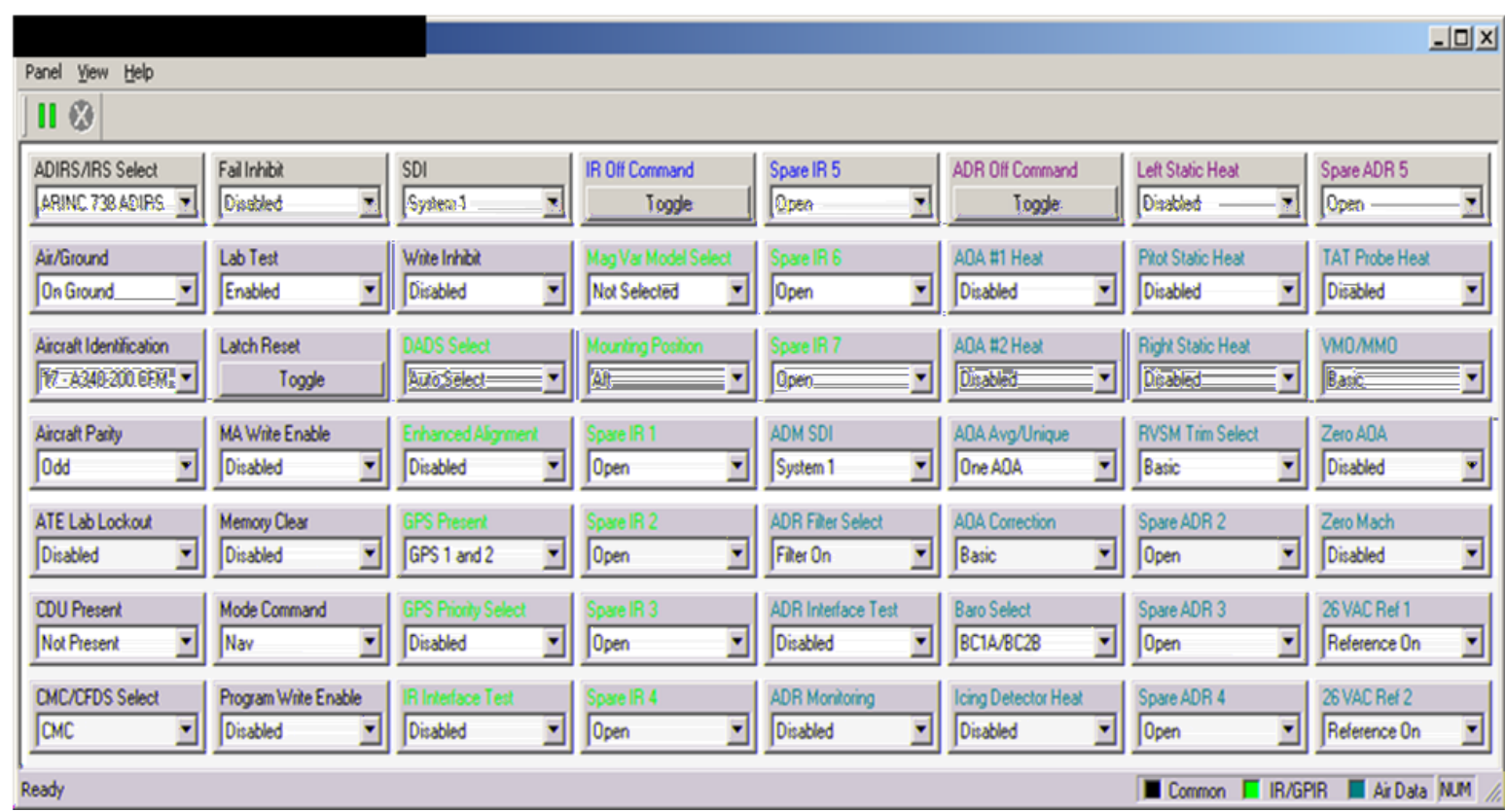

\section{FIGURE 3.1 INPUT DISCRETE VIRTUAL PANEL}

\begin{tabular}{|l}
\hline W Output Discretes - Virtual Panel \\
\hline Cmp Latch PS Latch \\
0
\end{tabular}

\section{FIGURE 3.2 OUTPUT DISCRETE VIRTUAL PANEL}

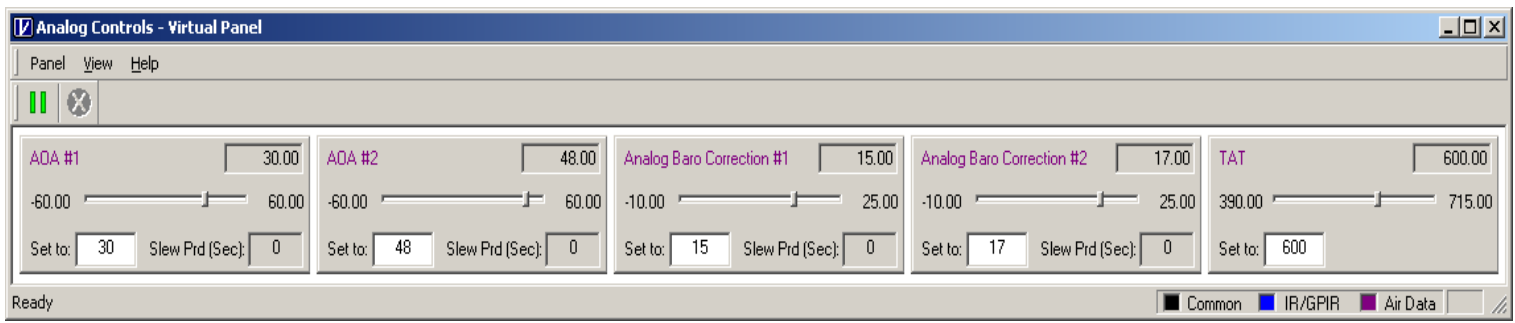

FIGURE 3.3 ANALOG CONTROLS VIRTUAL PANEL 


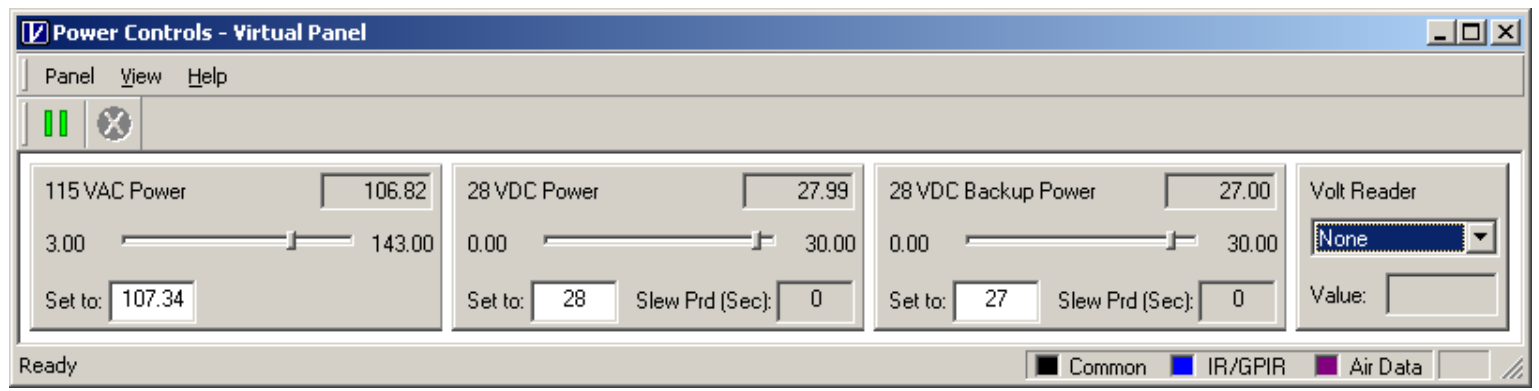

\section{FIGURE 3.4 POWER CONTROLS VIRTUAL PANEL}

\subsection{Applications for Software Verification}

\subsubsection{Mercury Interactive WinRunner}

WinRunner from Mercury Interactive Corporation (WinRunner) is a software application that supports the generation of automated test cases either through the capture playback method or by utilization of the built-in Test Scripting Language (TSL) to construct test procedures. This is an off-the-shelf product. WinRunner allows for custom functions to be created that can record "pass" or "fail" test steps.

\subsubsection{Customized Function Library}

When constructing on automated test, it is desireable to use functions to avoid repetition of many lines of code. See the appendix for a detailed code description of the current useable functions created specifically for avionics testing. These functions can be customized to manipulate data on any windows-based GUI. They were written using TSL. The verification procedures would typically be structured as shown in Figure 3.5 (the functions are shown in bold). 


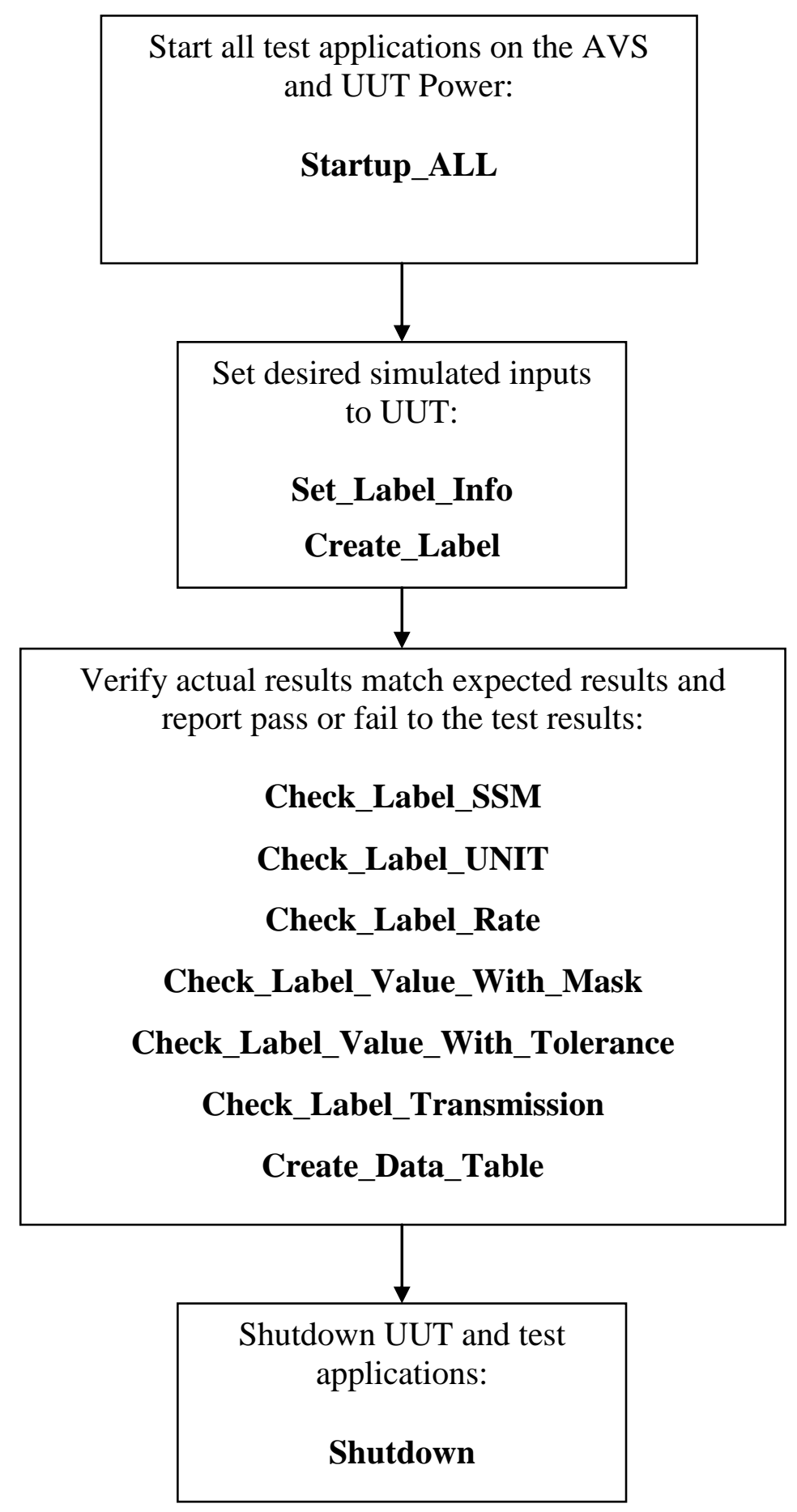

FIGURE 3.5 AUTOMATED TEST FLOW 


\subsection{Test Station Startup Functions}

The following list are functions used to start applications used for verification on the test station. The function "Startup_ALL" will open all the test applications and power on the UUT. The function "Add_to_Generator" will populate a custom function menu in WinRunner. This allows a user to insert a function and provides instructions. The other functions start an individual application in case not all of them are needed in a given test.

- Startup_ALL

- Startup_Virtual_Panels

- Startup_MIU

- Startup_ACO_Trans16

- Startup_ACO_Trans8

- Startup_ACO_Rec8

- Startup_ACO_Rec16

- Add_to_Generator

\subsection{Verification Test User Functions}

The functions listed in this section can be used to construct a test. A test will, in general, have one or more of these functions, which will report a pass or fail to the test results. The first two functions create or set an input label to a desired condition, while the other functions check the system output against expected results and report to the test results. The "Shutdown" function turns off the UUT and closes the test applications.

- Create_Label

- Set_Label_Info

- Check_Label_SSM

- Check_Label_Unit

- Check_Label_Rate

- Check_Label_Value_With_Mask

- Check_Label_Value_With_Tolerance

- Check_Label_Transmission

- Create_Data_Table

- Shutdown 


\subsection{ATP, EMI, and Environmental Test Functions}

These functions were developed to assist in qualification tests that are required of many aircraft systems. The Acceptance Test Procedure (ATP) is used to verify the stability of the system hardware. This can be done by developing embedded software that is only designed to exercise the hardware. Chapter 6 discusses these tests in more detail.

- ATP_Check_Receivers

- ATP_Check_Input

- ATP_Check_Resolvers

- ATP_Check_Output_Discretes

- EMI_Check_Receivers

- EMI_Check_Input

- EMI_Check_Resolver

- EMI_Check_Output_Discretes

\subsubsection{DOORS}

DOORS is a requirements management tool from IBM/Telelogic that is widely used throughout the aerospace industry. Requirements should have a defined structure that is used throughout a program. Having this defined structure allows for database manipulation of the requirements and even automatic generation of test cases for the requirements. The requirement attributes can be extracted from DOORS based on defined rules that have been incorporated into a windows-based extraction tool. The extraction is then loaded into a SQL tool database, and into the requirements section of the WinRunner tool used for traceability, test generation, test execution and results comparison. 


\subsubsection{System Requirements Extraction and Synchronization}

The extraction tool compares the previous extractions in the SQL database to the current extraction to determine requirements additions and deletions when synchronizing with WinRunner. If a requirement was deleted from DOORS, it would thus be deleted along with any traceability from WinRunner. If a requirement was added to DOORS since the last extraction and synchronization, the new requirement would be added to the WinRunner tool and traceability would be provided for each requirement. This qualification is performed on a preexisting SQL database such that additions and deletions are determined by a comparison from the last extraction and synchronization process.

\subsubsection{WinRunner Verification Test Shell Creation}

The synchronization between DOORS and WinRunner should only be performed on an as-needed basis. However, whenever changes are made to the requirements within DOORS that affect a test, another extraction and synchronization should be performed. When the synchronization is performed, all verification tests that were previously created by this process are deleted and new versions are created. A standard test description is loaded into each verification test created, and all the related requirement attributes are traced to this test based on rules within the extraction program. Finally, a test script is loaded into each test name created. This script consists of function calls that will create and execute the test based on the specific requirement ID. The script shell is standard for each verification test created but will differ based on the requirements for each specific test. 


\section{CHAPTER 4: VERIFICATION TEST RESULTS}

\subsection{WinRunner Verification Data Generation}

Once the verification test shell creation phase is complete, the tests can be scheduled and executed as a batch run or "test set". This enables many tests to be run with no operator effort or data entry. Therefore, tests can be run overnight, or throughout a weekend.

The tests can also be run individually. The WinRunner tool contains function scripts called from each created test shell. When the function calls are executed, the SQL database is queried for requirements associated with the requirement ID and the expected results for each test procedure.

\subsection{WinRunner Verification Test Results}

The automated test script is able to query the SQL tool database to return the requirements to be tested, along with the expected results for each verification test. Some requirements for each verification test may not be automatically testable. For those requirements, manual or other forms of verification tests are to be performed. Also, it is possible to have a hybrid test that is mostly automated, but will prompt a user for action. For example, the test may need to prompt a tester to change the pitch of a navigation system by a certain amount. 


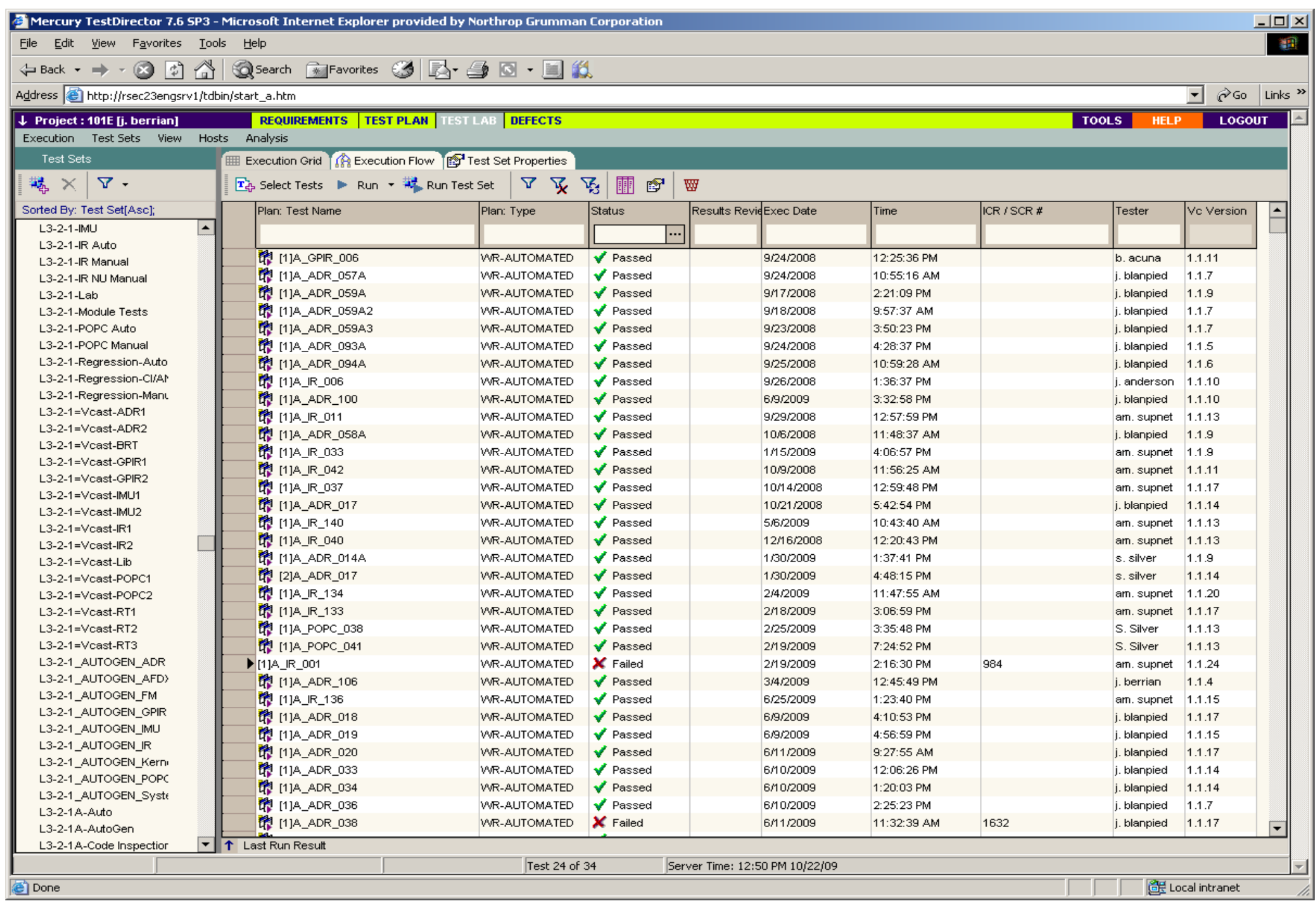

FIGURE 4.1 WINRUNNER VERIFICATION RESULTS IN A TEST SET 


\begin{tabular}{|c|c|c|c|c|}
\hline \multicolumn{4}{|c|}{ D Ele gotoons Iook whondow } & \multirow[t]{2}{*}{$\frac{-|0| x \mid}{-|\theta| x \mid}$} \\
\hline \multicolumn{4}{|c|}{ 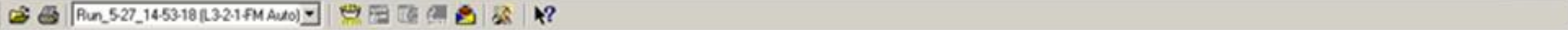 } & \\
\hline \multicolumn{5}{|c|}{ 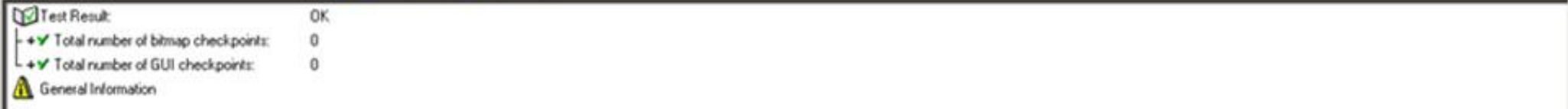 } \\
\hline Line Evert & Detalt & Rent: & Timo & 目 \\
\hline 18 thatinn & OMC_Nomulyado & in & 00000 & \\
\hline 111 titlep & 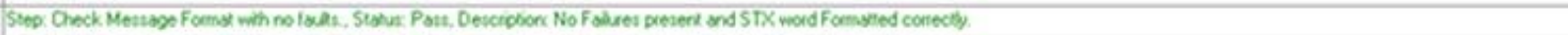 & - & 000211 & \\
\hline 149 thatep & 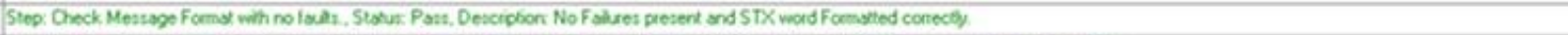 & - & $\infty 00255$ & \\
\hline 200 eliteo & 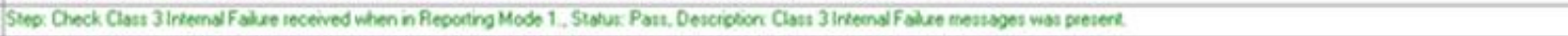 & - & 000416 & \\
\hline 2008 titieg & 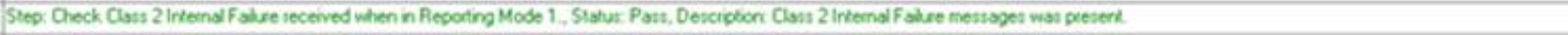 & - & 000417 & \\
\hline 265 enteep & 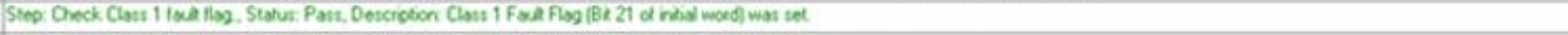 & - & 000428 & \\
\hline 256 (Litites & 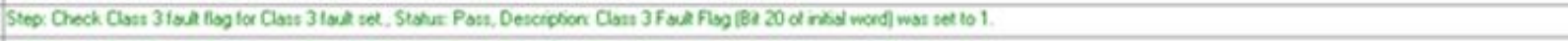 & - & 00043 & \\
\hline 300 enteleo & 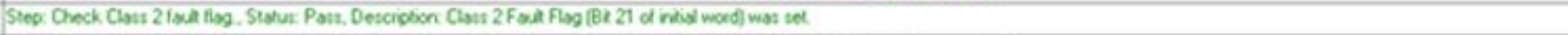 & - & 0005.14 & \\
\hline आL Litep & 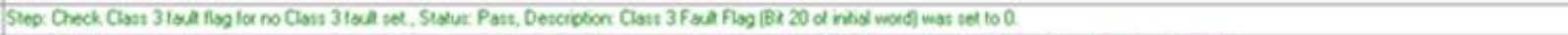 & - & $\infty 005.15$ & \\
\hline 342 etitep & 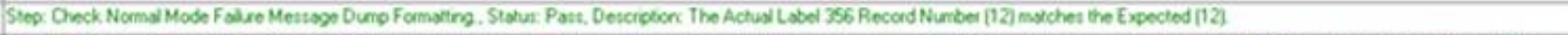 & - & 000549 & \\
\hline 353 titien & 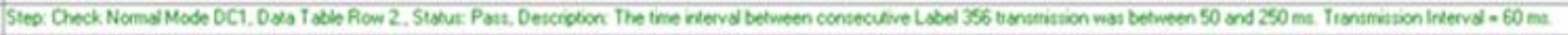 & - & 000551 & \\
\hline 363 Ltithe & 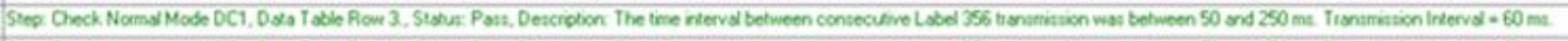 & - & 000551 & \\
\hline 363 Cites & 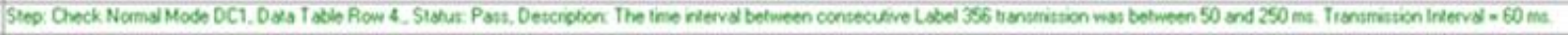 & - & 0005.52 & \\
\hline 353 Clites & 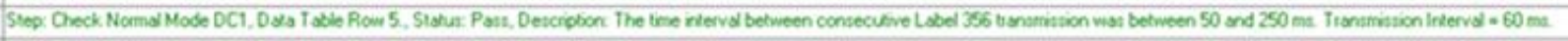 & - & 000554 & \\
\hline 363 totes & 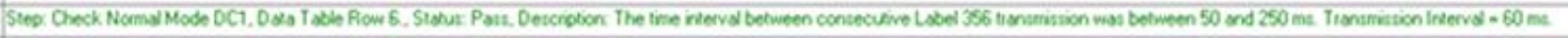 & - & 000556 & \\
\hline 363 thatep & 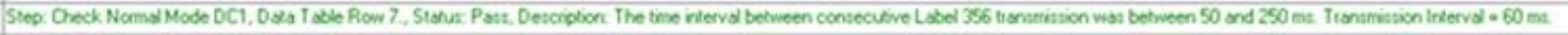 & - & 000557 & \\
\hline 363 thateo & 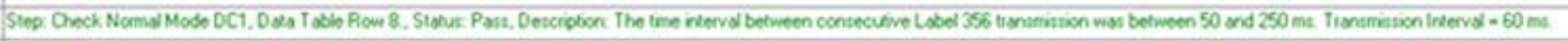 & - & 000558 & \\
\hline 353 tetsep & 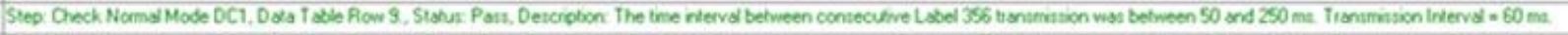 & - & 0005.59 & \\
\hline 363 Cliteo & 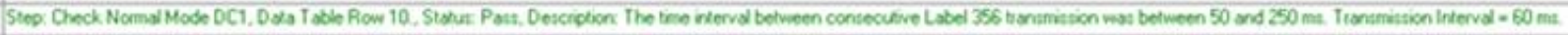 & - & 000600 & \\
\hline 363 C.titep & 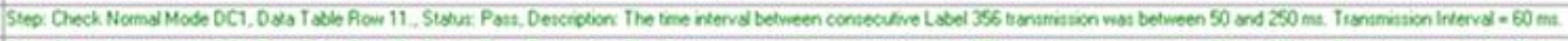 & - & 000601 & \\
\hline 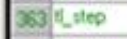 & 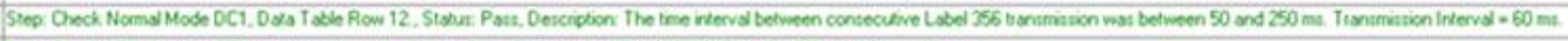 & - & $\infty 00600$ & \\
\hline 399 Custeo & 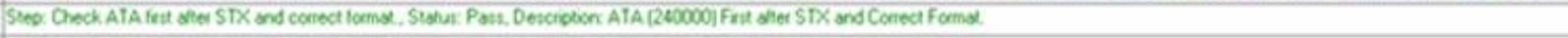 & - & $\infty 00605$ & \\
\hline 424 tistlep & 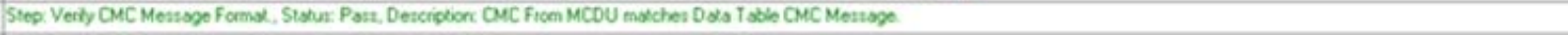 & - & 000607 & \\
\hline 408 CLsteteo & 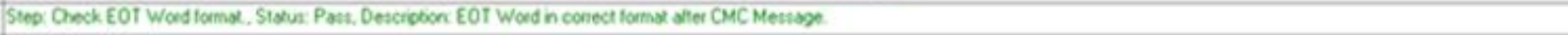 & - & 000609 & \\
\hline 460 thoos tun & OMC_Nomsulyoso & Dass & 000613 & \\
\hline Resoly & & Thetes & stesestatered. & \\
\hline
\end{tabular}

FIGURE 4.2 WINRUNNER VERIFICATION RESULTS FOR A SPECIFIC TEST 


\subsection{WinRunner Verification Test Execution}

When the test(s) complete execution, the results from the test run(s) are sent to the WinRunner database. Either the tests passed, where all actual results matched all expected results for each step, or one or more actual results did not match expected results, which results in a test fail status. 


\section{CHAPTER 5: TEST STATION QUALIFICATION PLAN}

The following sections discuss the steps to be performed in order to qualify the process and tools involved in the AVS. This is necessary for many Design Assurance Levels of DO-178B process guidelines.

\subsection{AVS Operational Requirements}

First, the operational requirements for the AVS need to be generated. This document should contain details on the process including the procedure, process diagrams and the function library listing used in the test process. The function library should be a large focus of this qualification plan.

\subsection{Function Library Compliance / Test}

The AVS Operational Requirements should document the function library scripts that are available to be used in any formal verification test procedure. Test cases will then created to verify the function library scripts correctly test the requirements and correctly report pass and fail results. A matrix is generated that relates each function to the test case(s) that verify it and the result of the test case(s). It will be shown that a function will correctly verify the requirement it is intended to test. This is done by inserting two types of input data to each test function; one resulting in a pass, and the other in a fail result. One would expect that a function records a "pass" if the actual result matches the expected result. It will also be shown that if the expected and actual result do not match, a "fail" is reported for that step.

\subsection{Test Coverage Compliance}

It also must verified that the verification process produces test cases for each requirement. This is necessary to show that the process did not generate too many or too 
few test cases. This is to be done on a sample of the verification tests in the high level software requirements that are representative of various characteristics of the UUT.

\subsection{Requirements Compliance}

A comparison should be made that compares the DOORS requirements database to the WinRunner database to show that an equal amount of requirements were autogenerated by the tool. There should be the same amount of requirements in both databases and should match exactly.

\subsection{Tool Qualification Summary}

A report will be generated that contains all the listed output(s) from each step of the qualification process in summary form. The report will also contain the developer of the report, date of report generation, function library version, requirements version, and all appropriate versions of the tools used. 


\section{CHAPTER 6: TEST STATION SCOPE AND GENERAL USES}

The hardware rack containing all the parts that comprise the test platform are designed to be mobile and universal. This presents a very significant enterprise advantage. A company could customize this standard platform to be compatible across many if not all programs. The test station software and hardware can be developed to communicate with any data bus, analog or digital resolvers, open/ground discretes, Ethernet protocols, etc.

\subsection{General Software Verification}

Automation is especially beneficial for required verification testing when it is applied to regression testing. Changes to software need to be tracked, controlled, and retested. As software is updated and released, these changes need to be analyzed to see which parts of the UUT need to be verified. This involves repeat testing, and automation reduces the time and cost of this effort.

\subsection{Hardware Acceptance and Environmental Testing}

At times in the design process, it is necessary to check the complete hardware configuration of the UUT. To this end, an Acceptance Test Procedure (ATP) can be performed. This is done to ensure all data pathways are in working order on a particular system. Generally, a comprehensive embedded software program can be written before the OFP is complete. This unique software program is designed to exercise all buses and paths of the hardware. For example, the UUT can be configured to send 0x5a5a5a on all buses and/or alternate open/ground discrete states. Once installed in the UUT, the automated test platform can be utilized to verify all hardware is responding appropriately.

The same software can be used for environmental testing of an aircraft system. Some tests induce Electromagnetic Interference (EMI) to try to "break" system hardware 
(e.g. check for bit switching on data buses, or state switching of input/output discretes).

As system hardware is generally ready prior to system software, these tests can be run earlier in a program lifecycle than software verification.

6.3 Functional Maintenance Testing

Similar to the ATP, functional testing can be performed to check production units after maintenance has been on a system.

6.4 Mobile Testing

As the automated test platform is portable, it can be used to record data while in flight test or drive test. It can also be taken to specialized testing facilities that conduct environmental stress testing. 


\section{CHAPTER 7: CONCLUSIONS}

The scope of this project was to propose a design for a portable, customizable, and maintainable automated test station. Without automation, software regression testing will require manual repetition of verification procedures. Furthermore, there is a learning curve involved with understanding manual test interfaces across different types of aircraft systems. With one upgradeable and customizable test station, a company can save time and money, and possibly win more contracts with lower bids.

Provided herein is such a test station design, along with test functions that allow for user-friendly verification test generation. The hardware and software design of the station can be upgraded or customized to interface with a number of aircraft systems that communicate using various protocols.

The implementation of an automated test station reduces human error associated with repeat testing. The function library can be qualified to perform the same with each test run and report passes and failures accordingly. Thousands of requirements can be exported into a SQL database and "fed" into the parameterized test script. Therefore, systems can be tested with deeper analysis in a shorter amount of time.

The test station described in this document is designed to be a "start-to-finish tester. It can be used for hardware verification (Acceptance Test Procedures), software/hardware integration, and system verification. The test station is portable and can be used in aircraft for flight testing, in vehicles for GPS and INS testing, and off-site environmental test laboratories. The AVS is a universal and customizeable verification test suite. 


\section{BIBLIOGRAPHY}

TestDirector User's Guide, Version 8.0

Mercury Interactive Corporation

Sunnyvalle, CA 94089

Copywright 2003

Advanced WinRunner 7.5

Mercury Interactive Corporation

Copywright 1994-2002

CEI-100/CEI-200/CEI-x20 User's Manual

Condor Engineering, Inc.

Santa Barbara, CA 93101

Copywright 1998-2001

Guide in Using ParaView, Version 5.0x

Aim $\mathrm{GmbH}$

Valley, NE 68064

Copywright 2003

Reference Manual AyI-FDX-2/4 AMC-FDX-2 Application Interface Libraty Version $7.2 \mathrm{x}$

AIM USA

Valley, NE 68064

Copywright 2001-2002

SCXI 1160 User's Manual

National Instruments Corp.

Austin, TX 78759

Copyright 1992, 1999

SCXI 1180/1181 User's Manual

Copyright 1991-1995

653x User's Manual

Copyright 1997, 2001

PXI-1011 Chassis User's Manual

Copyright 2000, 2002

SCXI-1127/1128 User's Manual

Copyright 1999, 2000 


\section{APPENDIX A: GLOSSARY}

\section{AC Alternating Current Voltage.}

ACO ARINC Communication Organizer. Test computer software application that can simulate ARINC inputs and receive outputs from the UUT.

AFDX Avionics Full Duplex. Ethernet based approach for avionics data transmission.

ARINC Aeronautical Radio, Incorporated. A major provider of data communication systems (both protocol types and hardware).

ATP Acceptance Test Procedure. A test performed to exercise all system hardware connections.

Auto-Gen Test Procedure Auto-Generation. A process used to automatically generate verification tests directly from the software high level requirements data base.

AVS Automated Verification Station. This can be developed to perform system level testing. This station provides all hardware and software necessary for performing system level testing either automatically or manually.

BITE Built-In-Test-Equipment. Tests developed and embedded in the system software. Used for detection and reporting of system errors.

DC Direct Current Voltage.

DOORS IBM / Telelogic software application. A database tool used to develop and maintain requirements. This is an off-the-shelf product.

GPS Global Positioning System. Almost all aircraft contain at least one GPS receiver which uses time mark data from at least 4 satellite sources to calculate global location and velocity.

GUI Graphic User Interface. This is how the operator interfaces with applications in a Windows operating system. The automated testing utility "learns" a GUI map of every Windows application used for testing.

WinRunner WinRunner from Mercury Interactive Corporation. A tool integrated with the test station computer that supports the generation of automated test cases either through the capture playback method or by utilization of the built-in Test Scripting Language (TSL) to construct test procedures. This is an off-the-shelf product.

INS Inertial Navigation System. Consisting of gyros (body rate data) and accelerometers (lateral movement), the INS, coupled with Kalman filtering, can predict location based on previous movements. 
I/O Input/Output. These are the signals going into and coming from the Unit Under Test (UUT). These signals can be analog or digital, discretes or status words, etc.

LRU Line Replaceable Unit. A sub-system of an aircraft. Examples include navigation systems, avionics displays, engine control systems.

MIU Memory Inspector Utility. A Windows-based application that can read and write UUT flash memory. Can be used to load operating software, control memory variables, record data, etc.

TSL Test Script Language. A coding language used to control test procedures and windows applications through the various GUIs.

UBC Universal Bus Communicator. A Windows-based application that can manage input and outputs (I/O) to and from the UUT.

UUT Unit Under Test. The system (black box) that has been designated for testing. 


\section{APPENDIX B: FUNCTION LIBRARY}

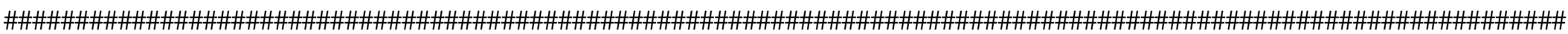

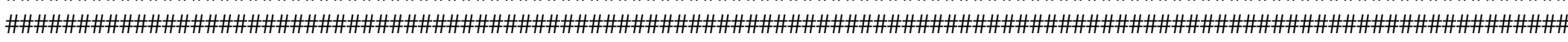
\# !!!THIS AREA CONTAINS THE TEST FUNCTION LIBRARY!!

\# Author: Joshua Berrian

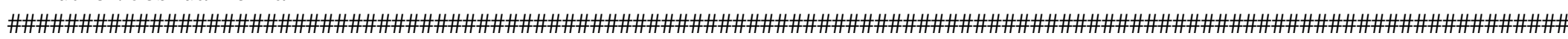
\# TEST STATION STARTUP FUNCTION LIST

\# Startup_Virtual_Panels(InputPanel)

Startup_MIU()

Startup_ACO_Trans16()

Startup_ACO_Trans8()

Startup_ACO_Rec8()

Startup_ACO_Rec16()

Startup_CMC(Function)

Startup_ALL(InputPanel, Function)

Add_to_Generator()

\section{\# USER FUNCTION LIST}

\# Check_Label_SSM(step, Function, Label, Expected_SSM, Expected_SSM2)

Check_Label_Unit(step, Function, Label, Expected_Unit)

Check_Label_Rate(step, Label, Function, Expected_Rate)

Check_Label_Value_With_Mask(step, Function, Label, Expected_Value, Mask)

Check_Label_Value_With_Tolerance(step, Function, Label, Expected_Value, Min_Acceptable_Value, Max_Acceptable_Value) Check_Label_Transmission(step, Function, Label, Expected_Transmission)

Set_Label_Info(Function, Port, Label, Value, SSM, Rate, Unit)

Shutdown(InputPanel)

Create_Data_Table(Table_Name, Folder_Path, num_columns, Column_Names)

Create_Label(Function, Port, Label, Name, Partition, SubFunction, Units, Value_Hex, Rate)

\# STATIC FUNCTION LIST

Close_DOS_CMC()

Bit16_to_Hex4 (Bit, out Hex)

Bit4_to_Hex1 (Bit, out Hex) 
\# ATP HARDWARE CHECKOUT FUNCTION LIST

ATP_Check_Receivers(Function)

ATP_Check_Input(Function)

ATP_Check_Resolvers()

ATP_Check_Output_Discretes

\section{\# EMI HARDWARE CHECKOUT FUNCTION LIST}

\# EMI_Check_Receivers(Function)

\# EMI_Check_Input(Function)

\# $\quad$ EMI_Check_Resolvers()

\# EMI_Check_Output_Discretes()

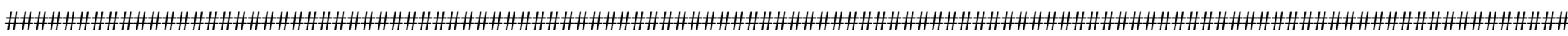

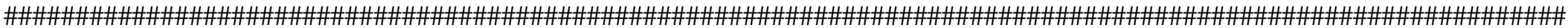
\#\#\#\#!!!ADDING USER FUNCTIONS TO FUNCTION GENERATOR!!!\#\#\#

public function Add_to_Generator()

auto func_desc;

\#Add enhanced tl_step to Function Generator

func_desc $=($ "This function divides test scripts into sections and inserts $\backslash r \backslash n "$ \&

"a status message in the test results. $|r| n "$ \&

"When WinRunner is connected to a TestDirector project, $\backslash r \backslash n " \&$

"the message is inserted to the TestDirector steps table $\backslash$ rnn" \&

"as well. \rın" \&

" arg1 is the name of the test step. $\operatorname{rr} \backslash n " \&$

" arg2 sets whether the step passed(PASS) or failed(FAIL). Ir $\backslash n "$ \&

" arg3 is a short explanation of the step. $|r| n " \&$

" arg4 is the expected result $\backslash \mathbf{r} \backslash n$ " \&

" arg5 is the actual result.");

generator_add_function("tl_step",func_desc, 5,

"Step Name", "type_edit","\"\"",

"Status", "select_list(PASS FAIL)", "PASS",

"Description", "type_edit","।"।"',

"Expected", "type_edit","\"\"",

"Actual","type_edit","\"\"'); 
\#Add Check_Label_SSM to Function Generator

func_desc $=($ "This function checks the expected SSM message to the $\backslash r \backslash n "$ \&

"actual and sends a PASS/FAIL message to the test results. $|r| n " \&$

" arg1 is the name of the test step.|rln" \&

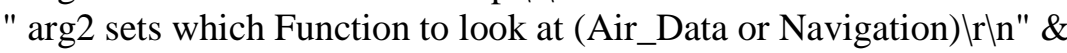

" arg3 is the Port channel. $|r| n "$ \&

" arg4 is the label to look at. Irln" \&

" arg5 is the expected SSM message, choose from: $|r| n "$ \&

" $\quad$ Failure Warning $\backslash r \backslash n "$ \&

" $\quad$ No Computed Dataไrın" \&

" Normal Operation $\backslash r \backslash n "$ \&

" arg6 is a second SSM for test pass (optional). Irln" \&

" arg7 is reserved BITE(leave blank).");

generator_add_function("Check_Label_SSM",func_desc, 7,

"step", "type_edit","।"|"",

"Function", "select_list(("Air_Datal" \"Navigation।")","\"Air_Datal"",

"Port", "type_edit", "',

"Label", "type_edit","",

"Expected_SSM", "select_list ( $(" F a i l u r e$ Warning।" \"No Computed Datal" \"Normal

Operation!")","।"Failure Warning|"",

"Expected_SSM2", "select_list(।"|"।"Failure Warning|"।"No Computed Datal" \"Normal

Operationl")","|"|"",

"BITE", "type_edit", "");

\#Add Check_Label_Unit to Function Generator

func_desc $=($ "This function checks the expected Unit to the actual|rın" \&

"and sends a PASS/FAIL message to the test results.Irln" \&

" arg1 is the name of the test step. $\operatorname{rrn} "$ \&

" arg2 sets which Function to look at (Air_Data or Navigation) $\backslash r\lfloor n "$ \&

" arg3 is the Port channel.|rın" \&

" arg4 is the label to look at.Irın" \&

" arg5 is the expected Unit, choose from:Irın" \&

" $\quad 1 \backslash r \backslash n " \&$ 
generator_add_function("Check_Label_Unit",func_desc, 5,

"step", "type_edit","।"I"",

"Function", "select_list(\"Air_Datal"।"Navigation\")","\"Air_Datal"",

"Port", "type_edit", "',

"Label", "type_edit","',

"Expected_Unit", "select_list(1 2 3)", "1");

\#Add Check_Label_Rate to Function Generator

func desc $=$ ("This function checks the expected Rate to the actuallrln" \&

"and sends a PASS/FAIL message to the test results. $|r| n "$ \&

" arg1 is the name of the test step. $\operatorname{lr} \mid n "$ \&

" arg2 sets which Function to look at (Air_Data or Navigation) $\backslash r \mid n " ~ \&$

" arg3 is the Port channel.Irln" \&

" arg4 is the label to look at..r.ın" \&

" arg5 is the expected Rate");

generator_add_function("Check_Label_Rate",func_desc, 5,

"step", "type_edit","।"|"",

"Function", "select_list(।"Air_Datal" \"Navigation\")","।"Air_Datal"",

"Port", "type_edit", "',

"Label", "type_edit","',

"Expected_Rate", "type_edit", "");

\#Add Check_Label_All_Ports to Function Generator

func desc $=($ "This function checks that a label Value is the $\operatorname{rln} " \&$

"same on all data buses within a certain tolerance. $|r| n " \&$

"(e.g +/- 0.1). Irln" \&

" arg1 is the name of the test step. Irın" \&

" arg2 sets which Function to look at (Air_Data or Navigation).।rın" \&

" arg3 are the ports (comma delimited). Ir $\backslash n "$ \&

" arg4 is the label to look at. Irln" \&

" arg5 is the Lower Tolerance. $|r| n " ~ \& ~$

" arg6 is the Upper Tolerance."); 
generator_add_function("Check_Label_All_Ports",func_desc, 6,

"step", "type_edit","।"|"",

"Function", "select_list(\"Air_Datal" \"Navigation।")","\"Air_Datal"",

"Ports", "type_edit", "I",|"'",

"Label", "type_edit","",

"Min_Tolerance", "type_edit", "',

"Max_Tolerance", "type_edit", "");

\#Add Check_Label_Value_With_Mask to Function Generator

func_desc $=($ "This function checks the expected label Value to the $\operatorname{rln} "$ \&

"actual and sends a PASS/FAIL message to the test results.|r|n" \&

" $\arg 1$ is the name of the test step.|r|n" \&

" arg2 sets which Function to look at (Air_Data or Navigation) $\backslash r\lfloor n " ~ \&$

" arg3 is the Port channel.|rın" \&

" arg4 is the label to look at.Irın" \&

" arg5 is the expected Value."

" arg6 is the mask (only bits with 1's are compared.)");

generator_add_function("Check_Label_Value_With_Mask",func_desc, 6,

"step", "type_edit","।"|"",

"Function", "select_list(\"Air_Datal" \"Navigation।")","\"Air_Datal"", "Port", "type_edit", "',

"Label", "type_edit","',

"Expected_Value", "type_edit", "I"।"",

"Mask", "type_edit", "।"|"');

\#Add Check_Label_Time_With_Tolerance to Function Generator

func_desc $=($ "This function checks the expected label time value to the $|r| n "$ \&

"actual and sends a PASS/FAIL message to the test results. Irln" \&

" arg1 is the name of the test step.|rln" \&

" arg2 sets which Function to look at (Air_Data or Navigation) $\backslash r \backslash n "$ \&

" arg3 is the Port channel.Irln" \&

" arg4 is the label to look at. Irın" \&

" arg5 is the expected Time. Ir $\backslash n "$ \& 
" arg6 is the Minimum Acceptable Time.Irın" \&

" arg7 is the Maximum Acceptable Time.");

generator_add_function("Check_Label_Time_With_Tolerance",func_desc, 7,

"step", "type_edit","।"।"",

"Function", "select_list(\"Air_Datal"।"Navigation।")","|"Air_Datal"",

"Port", "type_edit", "',

"Label", "type_edit","",

"Expected_Time", "type_edit", "\"00:00:00।"',

"Min_Time", "type_edit", "\"00:00:00\"",

"Max_Time", "type_edit", "।"00:00:00।"");

\#Add Check_Label_Date_With_Tolerance to Function Generator

func_desc $=($ "This function checks the expected label date value to the $\operatorname{rln} "$ \&

"actual and sends a PASS/FAIL message to the test results. Irln" \&

" $\arg 1$ is the name of the test step.Irln" \&

" arg5 is the expected Date. $|r| n "$ \&

" arg6 is the Minimum Acceptable Date.Irln" \&

" $\arg 7$ is the Maximum Acceptable Date.");

generator_add_function("Check_Label_Date_With_Tolerance",func_desc, 4,

"step", "type_edit","।"|"",

"Expected_Date", "type_edit", "I"MM\DD $\backslash Y Y \backslash "$ ",

"Min_Date", "type_edit", "I"MM\DD $\backslash Y Y \backslash "$ ",

"Max_Date", "type_edit", "I"MM\DDIYY।"");

\#Add Check_Label_Value_With_Tolerance to Function Generator

func_desc $=($ "This function checks the expected label Value to the $\backslash r \backslash n "$ \&

"actual and sends a PASS/FAIL message to the test results. Irln" \&

" $\arg 1$ is the name of the test step.|r|n" \&

" arg2 sets which Function to look at (Air_Data or Navigation) $)$ rln" \&

" arg3 is the Port channel.|rın" \&

" arg4 is the label to look at. Irın" \&

" arg5 is the expected Value.|r|n" \&

" arg6 is the Minimum Acceptable Value.|rın" \& 
" $\arg 7$ is the Maximum Acceptable Value.");

generator_add_function("Check_Label_Value_With_Tolerance",func_desc, 7,

"step", "type_edit","।"|"",

"Function", "select_list(\"Air_Datal"।"Navigation।")","\"Air_Datal"",

"Port", "type_edit", "',

"Label", "type_edit","",

"Expected_Value", "type_edit", "",

"Min_AcceptableValue", "type_edit", "',

"Max_Acceptable_Value", "type_edit", "");

\#Add Check_Label_Transmission to Function Generator

func_desc $=($ "This function checks the expected Label Tramission to thelrın" \&

"actual and sends a PASS/FAIL message to the test results.Irı" \&

" $\arg 1$ is the name of the test step.|r|n" \&

" arg2 sets which Function to look at (Air_Data or Navigation) $\backslash r\lfloor n " ~ \&$

" arg3 is the Port channel.|rın" \&

" arg4 is the label to look at. Irın" \&

" arg5 is the expected Tansmission Value. Irln" \&

" Not Transmitted $\quad$ rın" \&

" Transmitted");

generator_add_function("Check_Label_Transmission",func_desc, 5,

"step", "type_edit","l"|"",

"Function", "select_list(\"Air_Datal" \"Navigation।")","।"Air_Datal"",

"Port", "type_edit", "",

"Label", "type_edit","",

"Expected_Transmission", "select_list(\"Not Transmitted।" ।"Transmitted।")","।"Not Transmitted।"");

\#Add Check_Action_Code to Function Generator

func_desc $=$ ("This function checks the expected Action Code to the $r$ rln" \&

"actual and sends a PASS/FAIL message to the test results. Irın" \&

" $\arg 1$ is the name of the test step. $\operatorname{lr} \backslash n "$ \&

" arg2 is the expected Action Code Number"); 
generator_add_function("Check_Action_Code",func_desc, 2,

"step", "type_edit","|"|"",

"ActionCode", "type_edit","|"|"");

\#Add Set_Label_Info to Function Generator

func_desc $=($ "This function allows the user to update label $\backslash r \backslash n "$ \&

"information on the ACO Transmitter pages. Leave $|r| n "$ \&

"args 4, 5, 6, 7, or 8 blank if no new update.|rın" \&

" arg1 is the function of the label.Irln" \&

" arg2 is the ACO Port Number.Irın" \&

" arg3 is the label to update. $|r| n " \&$

" arg4 is the new Value of the label if in Eng units. $|r| n "$ \&

" arg5 is the new Value of the label if in Hex units.|rın" \&

" arg6 is the new SSM value.|rln" \&

" arg7 is the new transmission Rate. Irln" \&

" arg8 is the new Unit");

generator_add_function("Set_Label_Info",func_desc, 8,

"Function", "select_list(\"Air_Datal"।"Navigation\")","\"Air_Datal"",

"Port", "type_edit", "',

"Label", "type_edit", "",

"Value_Eng", "type_edit", "",

"Value_Hex", "type_edit", "।"|"",

"SSM", "select_list (\"Discretel" \"Normal Operation\" \"No Computed Datal" \"Interface Test।"।"Failure

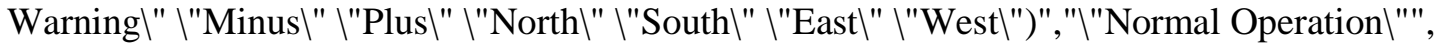

"Rate", "type_edit", "",

"Unit", "select_list(1 2 3)", "1");

\#Add Shutdown to Function Generator

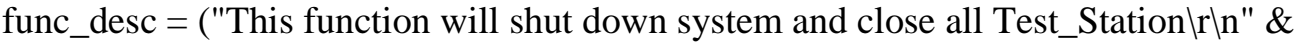

"applications. Irln" \&

" arg1 is which Input Panel you are using. Irln" \&

" Input Discretes - System1 - Virtual Panel\rın" \&

" Input Discretes - System2 - Virtual Panel\r|n" \&

" Input Discretes - System3 - Virtual Panel"); 
generator_add_function("Shutdown",func_desc, 1,

"InputPanel", "select_list(\"Input Discretes - System1 - Virtual Panell"।"Input Discretes - System2 -

Virtual Panel।"।"Input Discretes - System3 - Virtual Panell")", "।"Input Discretes - System2 - Virtual Panell"');

\#Add Startup_MIU to Function Generator

func_desc $=($ "This function will start the MIU application. Irın" \&

"Leave the Build_Number variable blank if you want to default $\backslash r \mid n "$ \&

" to the previous build setting in MIU.|r|n" \&

" arg1 is the Build you are using.|rln" \&

" arg2 is the RAM or ROM build choice.");

generator_add_function("Startup_MIU",func_desc, 2,

"Build_Number", "type_edit", "।"।"",

"RAM_ROM", "select_list(\"RAM_Program.mapl" ।"ROM_Program.mapl")",

"I"ROM_Program.mapl"');

\#Add MIU_Page to Function Generator

func_desc $=($ "This function will load a saved MIU page. $|r| n " \&$

"The page must be saved under Common. $|r| n " \&$

" arg1 is the User Name Directory.Irın" \&

" arg2 is the Page Name.");

generator_add_function("MIU_Page",func_desc, 2,

"Directory", "type_edit", "।"|"",

"Page_Name", "type_edit", "|"|"');

\#Add Create_Data_Table to Function Generator

func desc $=($ "This function will create a new excel file to a $|r| n " \&$

"specified folder path. Irln" \&

" arg1 new table name. Irın" \&

" arg2 is the folder location path - USE DOUBLE SLASHES (IIII). Irln" \&

" arg3 is the number of columns to make. Irln" \&

" arg4 is the list of the names of the columns - comma delimited."); 
generator_add_function("Create_Data_Table",func_desc, 4,

"Table_Name", "type_edit","|"|"",

"Folder_Path", "type_edit", "।"|"",

"num_columns", "type_edit", "",

"Column_Names", "type_edit", "।", , , , , , , ,"');

\#Add Create_Label to Function Generator

func_desc $=$ ("This function allows the user to create a label $\backslash r \backslash n " \&$

"in the standard Air_Data or Navigation ACO Pages. Leave last Three $\backslash r\lfloor n " ~ \&$

"args $(8,9,10)$ alone if creating receiver label only. $|r| n " ~ \&$

" arg1 is the Label Designation (Tx or Rx). $\operatorname{rr} \mid n "$ \&

" arg2 is the ACO Port Number.Irı" \&

" arg3 is the label to create. $|r| n "$ \&

" arg4 is the Name of the new label.ırın" \&

" arg5 is the partition using the data. $|r| n "$ \&

" arg6 is the subfunction of the label.|r|n" \&

" arg7 are the units.|r|n" \&

" arg8 is the hex value (bits 10-9 are the Unit). Irln" \&

" arg9 is the rate. $\operatorname{lr} \mid n "$ \&

" arg10 is the Unit.");

generator_add_function("Create_Label",func_desc, 10 ,

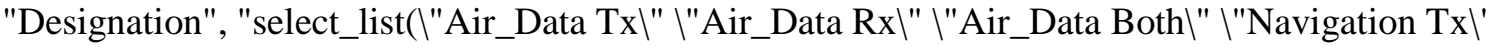

।"Navigation Rx \" \"Navigation Bothl")","\"Air_Data Rx।"",

"Port", "type_edit", "",

"Label", "type_edit", "",

"Name", "type_edit", "I"l"",

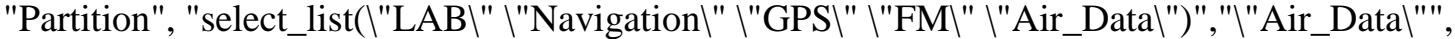

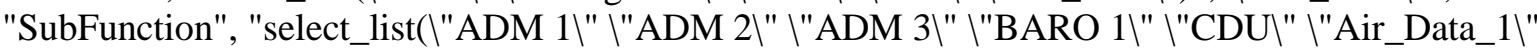

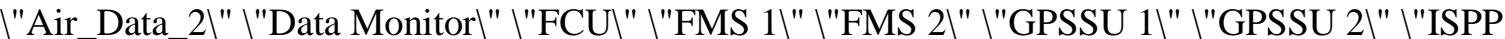

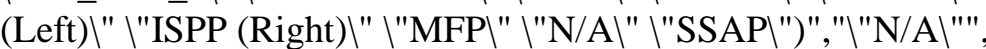

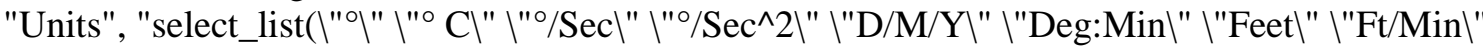

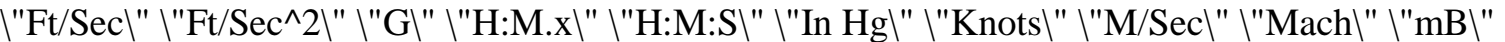

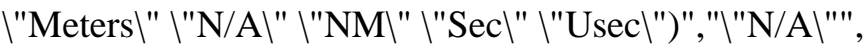

"Value_Hex", "type_edit", "|"|"",

"Rate", "type_edit", "", 
\#Add Add_Label to Function Generator

func_desc $=($ "This function allows the user to add a label $\backslash r \mid n " ~ \&$

"in the standard Air_Data or Navigation ACO Pages.Irln" \&

" arg1 is the Label Designation (Tx or Rx). $\operatorname{lr} \backslash n "$ \&

" arg2 is the ACO Port Number.Irın" \&

" arg3 is the label to create.");

generator_add_function("Add_Label",func_desc, 3,

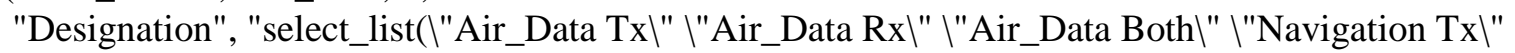

।"Navigation Rx।" \"Navigation Bothl")","|"Air_Data Rx।"",

"Port", "type_edit", "',

"Label", "type_edit", "");

\#Add Round to Function Generator

func_desc $=($ "This function will return a Rounded Number for a given $\backslash r\lfloor n " ~ \&$

"number of decimal places. Irın" \&

" $\arg 1$ is the Original Number.|rın" \&

" arg2 is the Decimal Places. Irln" \&

" arg3 is the Rounded Number returned.");

generator_add_function("Round",func_desc, 3,

"Number", "type_edit","",

"Decimal_Places", "type_edit", "",

"Rounded_Number", "type_edit", "Rounded_Number");

\#Add MIU shut down system and wait to Function Generator

func_desc = ("This function will shut down MIU.");

generator_add_function("MIU_Shutdown",func_desc, 0);

\#Add Shut down system and wait to Function Generator

func_desc = ("This function will shut down the system and wait for power off."); generator_add_function("Power_Off",func_desc, 0);

\#Add MIU_Wait to Function Generator

func_desc $=$ ("This function will wait for the screen to refresh."); 
generator_add_function("MIU_Wait",func_desc, 0);

\#Add StrToInt to Function Generator

func_desc $=$ ("This function will convert a Hex string to an integer.");

generator_add_function("StrToInt",func_desc, 2,

"strIn", "type_edit", "",

"IntOut", "type_edit", "IntOut");

\#ADD FUNCTION CATEGORY TO GENERATOR and ADD USER FUNCTIONS TO THAT CATEGORY generator_add_category("Custom");

generator_add_function_to_category("Custom","Check_Label_SSM");

generator_add_function_to_category("Custom","Check_Label_Unit");

generator_add_function_to_category("Custom","Check_Label_Rate");

generator_add_function_to_category("Custom","Check_Label_All_Ports");

generator_add_function_to_category("Custom","Check_Label_Value_With_Mask");

generator_add_function_to_category("Custom","Check_Label_Time_With_Tolerance");

generator_add_function_to_category("Custom","Check_Label_Date_With_Tolerance");

generator_add_function_to_category("Custom","Check_Label_Value_With_Tolerance");

generator_add_function_to_category("Custom","Check_Label_Transmission");

generator_add_function_to_category("Custom","Check_Action_Code");

generator_add_function_to_category("Custom","Set_Label_Info");

generator_add_function_to_category("Custom","Shutdown");

generator_add_function_to_category("Custom","Startup_ALL");

generator_add_function_to_category("Custom","Create_Data_Table");

generator_add_function_to_category("Custom","Create_Label");

generator_add_function_to_category("Custom","Add_Label");

generator_add_function_to_category("Custom","Startup_MIU");

generator_add_function_to_category("Custom","MIU_Page");

generator_add_function_to_category("Custom","Round");

generator_add_function_to_category("Custom","MIU_Wait");

generator_add_function_to_category("Custom","Power_Off");

generator_add_function_to_category("Custom","MIU_Shutdown");

\}

generator_add_function_to_category("Custom","StrToInt");

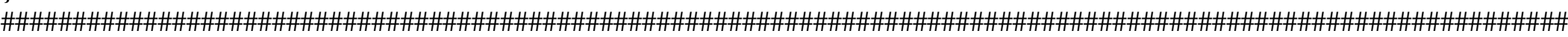

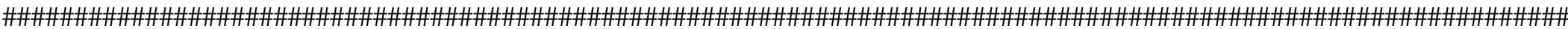

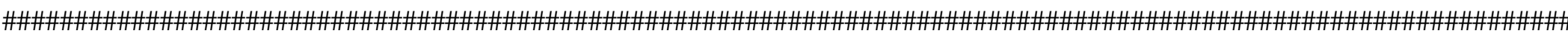


\#\#\#!!!STARTUP FUNCTIONS!!!\#\#\#\#

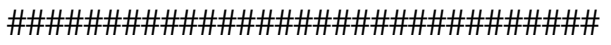

\#This sunction will start all Applications necessary for Test_Station use

public function Startup_ALL(InputPanel, Function)

\{

Startup_Virtual_Panels (InputPanel);

Startup_ACO_Trans16();

Startup_ACO_Trans8();

Startup_ACO_Receiver16();

Startup_ACO_Receiver8();

\}

Startup_MIU("", "");

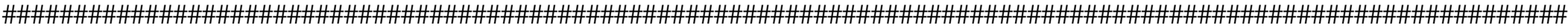

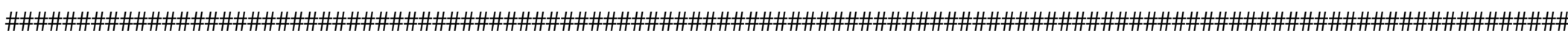
\#This function will startup all virtual panels if not already invoked.

public function Startup_Virtual_Panels (InputPanel)

\{

auto value;

auto i;

switch(InputPanel)

\{

case "Input Discretes - System1 - Virtual Panel":

if (win_exists("Input Discretes - System2 - Virtual Panel",0)==E_OK) win_close("Input Discretes - System2 - Virtual Panel");

if (win_exists("Input Discretes - System3 - Virtual Panel",0)==E_OK) win_close("Input Discretes - System3 - Virtual Panel");

break;

case "Input Discretes - System2 - Virtual Panel":

if (win_exists("Input Discretes - System1 - Virtual Panel",0)==E_OK) win_close("Input Discretes - System1 - Virtual Panel");

if (win_exists("Input Discretes - System3 - Virtual Panel",0)==E_OK) win_close("Input Discretes - System3 - Virtual Panel");

break;

case "Input Discretes - System3 - Virtual Panel":

if (win_exists("Input Discretes - System2 - Virtual Panel",0)==E_OK) 
win_close("Input Discretes - System2 - Virtual Panel");

if (win_exists("Input Discretes - System1 - Virtual Panel",0)==E_OK)

break;

win_close("Input Discretes - System1 - Virtual Panel");

\#\#\#\#\#\#\#\#\#\#

\# Initialize Input Discretes

if (win_exists (InputPanel, 1)==E_OK) \{\}

else

\{

if(win_exists("Select a Panel...", 1)==E_OK) \{\}

else

invoke_application("Virtual Panel.exe","',,"Virtual Panel",SW_SHOW);

\# Select a Panel..

set_window ("Select a Panel...", 10);

switch(InputPanel)

\{

case "Input Discretes - System1 - Virtual Panel":

list_select_item ("ListBox", "Input Discretes - System1");

break;

case "Input Discretes - System2 - Virtual Panel":

list_select_item ("ListBox", "Input Discretes - System2");

break;

case "Input Discretes - System3 - Virtual Panel":

list_select_item ("ListBox", "Input Discretes - System3");

break;

\}

button_press ("OK");

set_window(InputPanel, 5);

wait(2);

obj_wait_info("Status Bar","value","Ready",60); 
set_window(InputPanel, 2);

win_move (InputPanel, 1307, 159);

\#\#\#\#\#\#\#\#\#\#

\# Initialize Output Discretes

if (win_exists("Output Discretes - Virtual Panel", 1)==E_OK) \{\}

else

\{

if(win_exists("Select a Panel...", 1)==E_OK) \{\}

else

invoke_application("Virtual Panel.exe","',"Virtual Panel",SW_SHOW);

\# Select a Panel...

set_window ("Select a Panel...", 10);

list_select_item ("ListBox", "Output Discretes"); \# Item Number 8;

button_press ("OK");

\# Output Discretes - Virtual Panel

set_window ("Output Discretes - Virtual Panel", 7);

win_move ("Output Discretes - Virtual Panel", 1511, 1);

\#\#\#\#\#\#\#\#\#

\# Initialize Analog Controls

if (win_exists("Analog Controls - Virtual Panel", 1)==E_OK) \{\}

else

if(win_exists("Select a Panel...", 1)==E_OK) \{\}

else

invoke_application("Virtual Panel.exe","","Virtual Panel",SW_SHOW);

\# Select a Panel...

set_window ("Select a Panel...", 10);

obj_mouse_dbl_click("ListBox",1,1,LEFT);

wait(1);

for $(\mathrm{i}=0 ; \mathrm{i}=1 ; \mathrm{i}++)$

if $($ win_exists("Virtual Panel",2) == E_OK) 


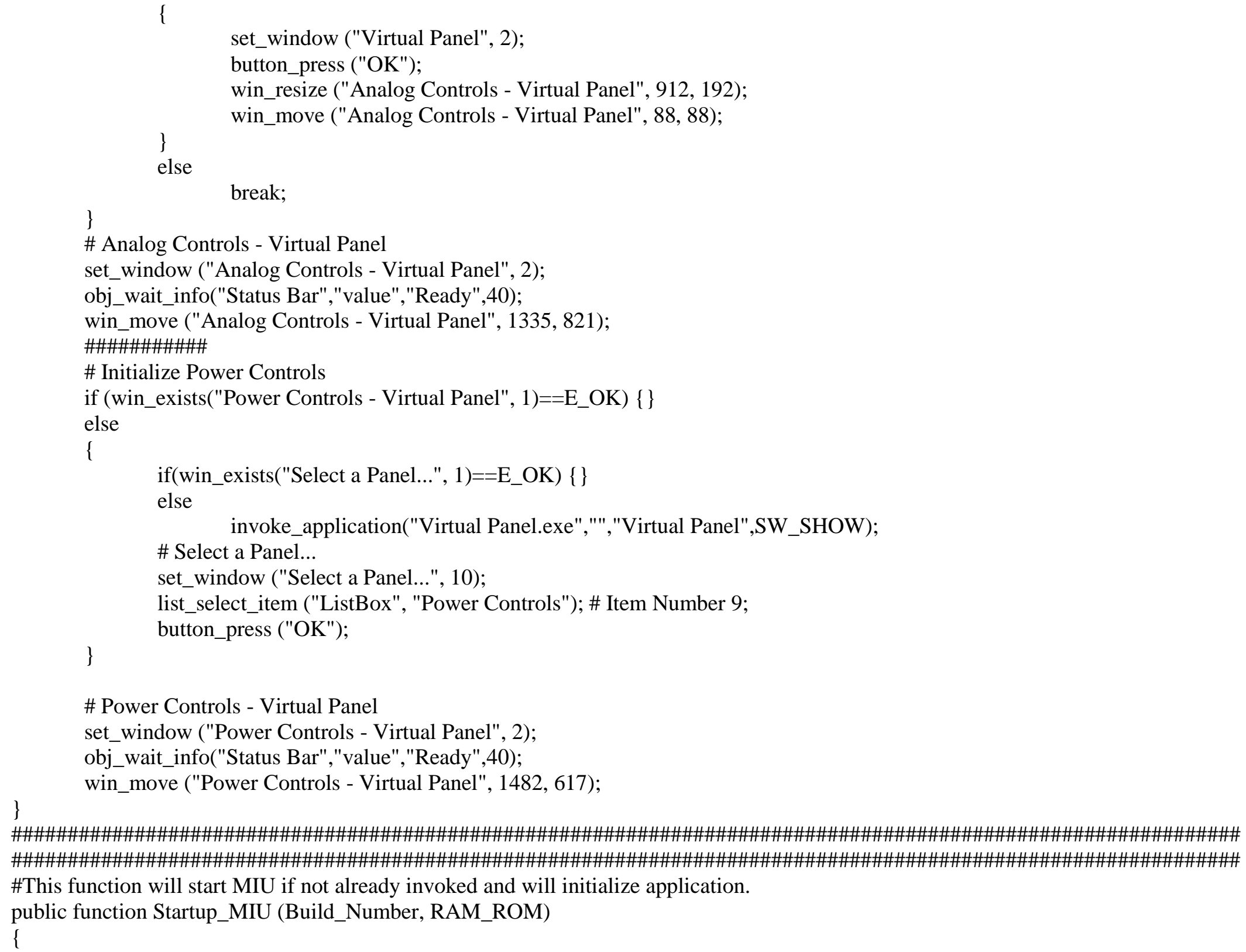


auto computer;

auto board;

auto rx;

auto tx;

auto array[];

if (win_exists("MIU",0)==E_OK)

return;

invoke_application("MIU.exe","'","MIU",SW_SHOW);

if(win_exists("ARINC Setup", 5)!=E_OK)

\{

\# User Information

set_window ("User Information", 3);

\}

button_press ("OK");

\section{\# ARINC Setup}

set_window ("ARINC Setup", 2)

obj_get_text("Board Select", board);

split(board, array, " ");

board = array[1];

obj_get_text("Rx Port Select", rx);

split(rx, array, " ");

rx = array[1];

obj_get_text("Tx Port Select", tx);

split(tx, array, " ");

$\mathrm{tx}=\operatorname{array}[1]$;

if (board $==0$ )

list_select_item ("Board Select", "1");

if ( $\mathrm{rx} !=1)$

list_select_item ("Rx Port Select", "\#0");

if $(\mathrm{tx} !=11)$

list_select_item ("Tx Port Select", "\#10");

button_press ("OK"); 


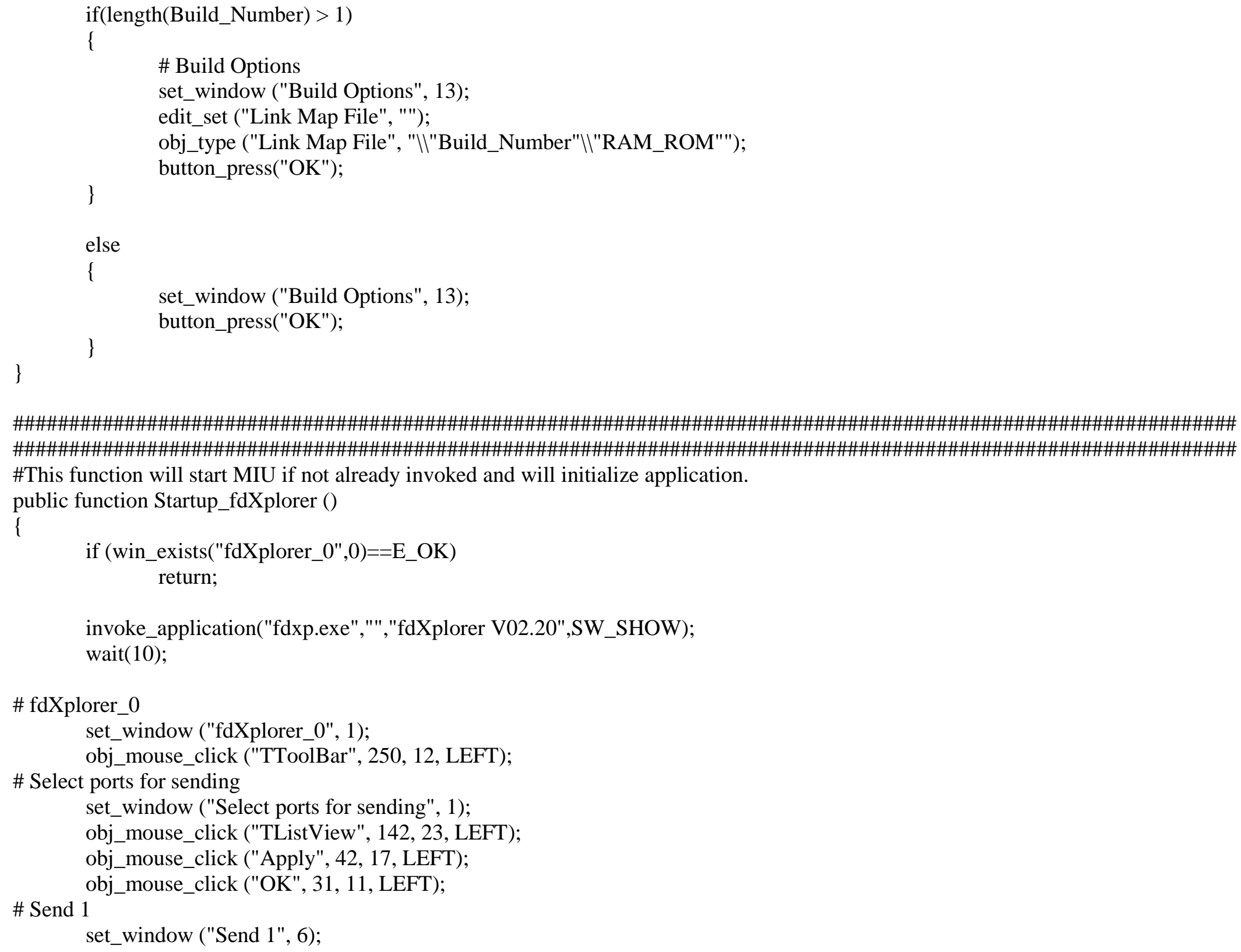


obj_mouse_click ("TToolBar", 7, 11, LEFT);

\# Import send setup file

set_window ("Import send setup file", 2);

obj_type ("File name:","<kCtrl_L-v>");

edit_set ("File name:","");

button_press ("Open");

\}

button_press("Start");

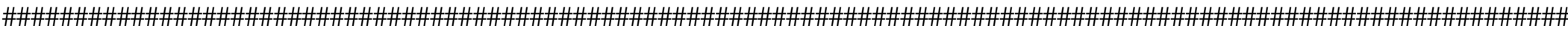

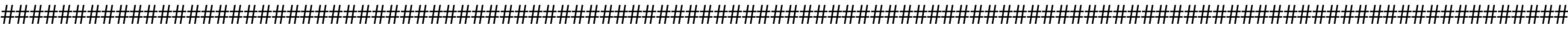

public function MIU_Page(Directory, Page_Name)

if (win_exists $($ Page_Name,0)==E_OK)

return;

\section{\# MIU}

set window ("MIU", 2);

toolbar_button_press ("ToolbarWindow32", "\#1");

\# Load Page from Database

set_window ("Load Page from Database", 5);

wait(1);

list_select_item ("User Name", Directory); \# Item Number 6;

list_select_item ("ListBox", Page_Name); \# Item Number 0;

button_press ("Open");

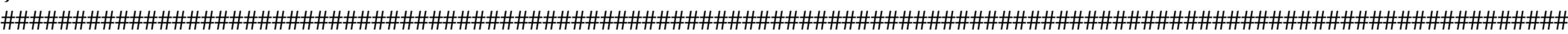

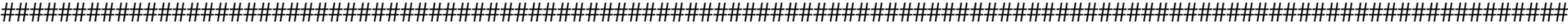
\#These functions will start Universal Arinc Testor for the following:

\# $\quad 16$ Channel Transmitter (Navigation) - "Test_Station Transmitter"

\# 8 Channel Transmitter (Air_Data) - "Air_Data Unit 1", "Air_Data Unit 2", "Air_Data Unit 3"

\# $\quad 16$ Channel Receiver (Navigation) - "Navigation Receiver"

\# 8 Channel Receiver (Air_Data) - "Air_Data Receiver"

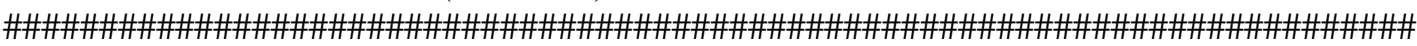

\#This function will start up 3 Air_Data Transmitter pages (1 Page per Unit)

public function Startup_ACO_Trans8 () 
auto i;

auto value;

auto computer;

\#Check and start 8 channel transmitter

for $(\mathrm{i}=1 ; \mathrm{i}<4 ; \mathrm{i}++)$

\{

if (win_exists("ACO - Air_Data Unit " i, 1)==E_OK) return;

\}

invoke_application("ACO.exe","',"'",SW_SHOW);

\# Select an ARINC board

set_window ("Select an ARINC board", 6);

list_select_item ("SysListView32", "0"); \# Item Number 0;

button_press ("OK");

\# ACO_1

set_window ("ACO_1", 2);

button_press ("Yes");

\section{\# Setup Page}

set_window ("Setup Page", 2);

button_press ("Load");

set_window ("Open", 7);

edit_set ("File name:", "");

edit_set ("File name:", "Setup.aco");

button_press ("Open_2");

for $(\mathrm{i}=1 ; \mathrm{i}<=4 ; \mathrm{i}++)$

\{

if(win_exists("ACO_1",2)==E_OK)

\{

\section{\# ACO_1}

set_window ("ACO_1", 2);

button_press ("OK");

\# Setup Page

set_window ("Setup Page", 2);

button_press ("Load"); 


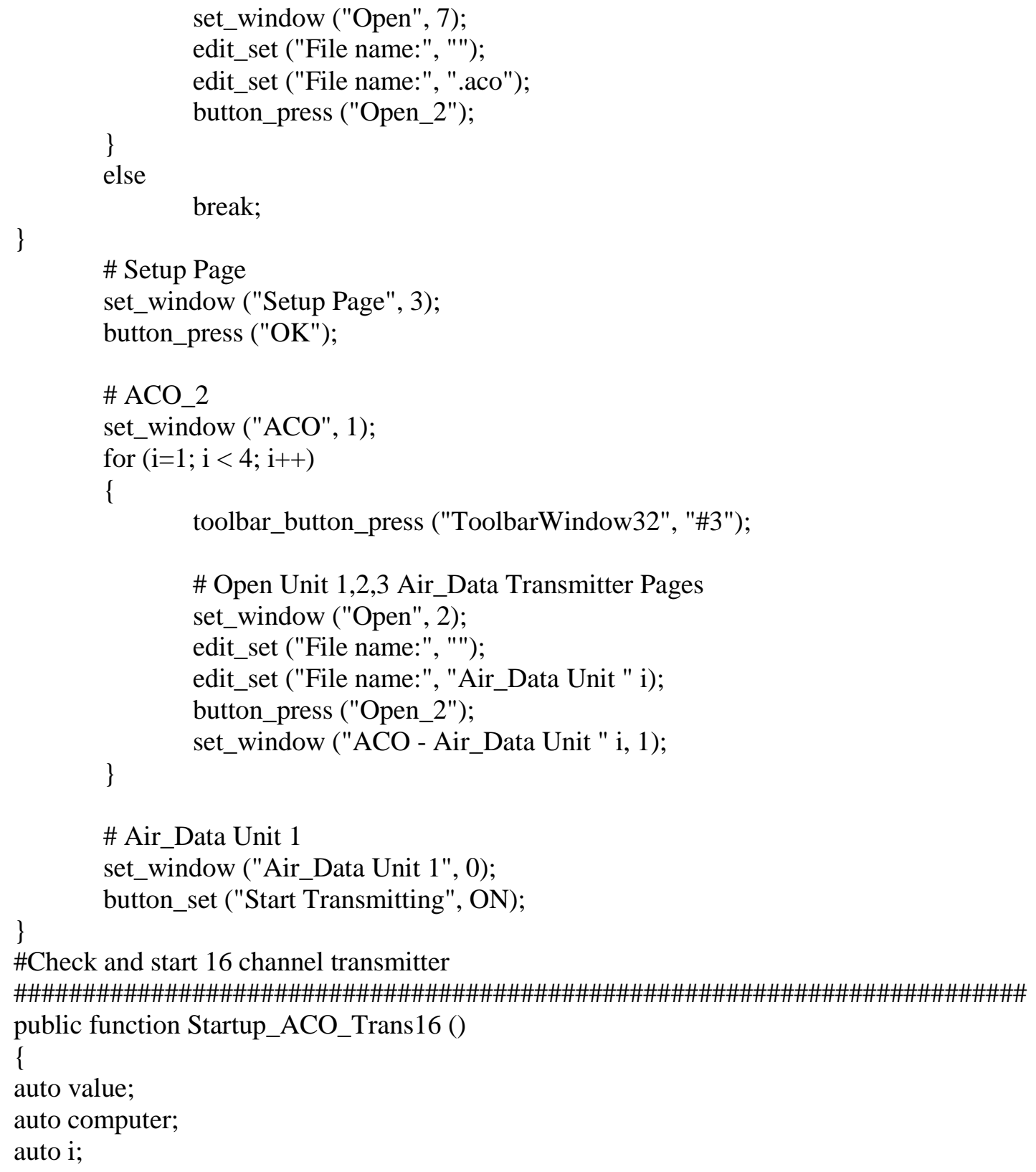


if (win_exists("ACO - Test_Station Transmitter", 0)==E_OK) return;

invoke_application("I\ACOI\ACO.exe","","',SW_SHOW);

\# Select an ARINC board

set_window ("Select an ARINC board", 4);

list_select_item ("SysListView32", "1"); \# Item Number 0;

button_press ("OK");

\# ACO_1

set_window ("ACO_1", 2);

button_press ("Yes");

\section{\# Setup Page}

set_window ("Setup Page", 2);

button_press ("Load");

\# Open

set_window ("Open", 2);

edit_set ("File name:", "");

edit_set ("File name:", "Test_Station Transmitter.aco");

button_press ("Open_2");

for $(i=1 ; i<=4 ; i++)$

if(win_exists("ACO_1",2)==E_OK)

\{

\# ACO_1

set_window ("ACO_1", 2);

button_press ("OK");

\# Setup Page

set_window ("Setup Page", 2);

button_press ("Load");

set_window ("Open", 7);

edit_set ("File name:", "");

edit_set ("File name:", "Test_Station Transmitter.aco");

\}

button_press ("Open_2");

else 


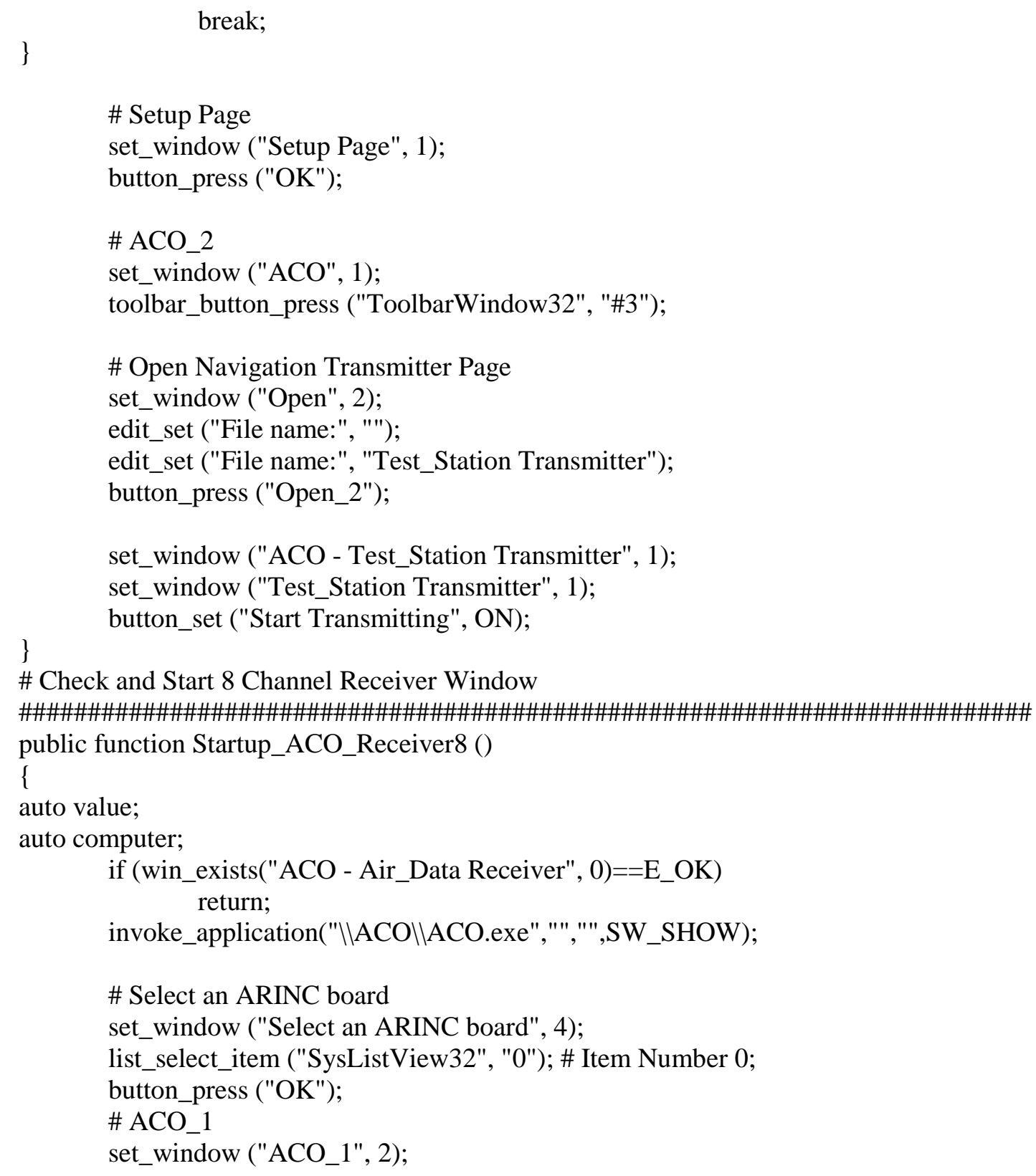




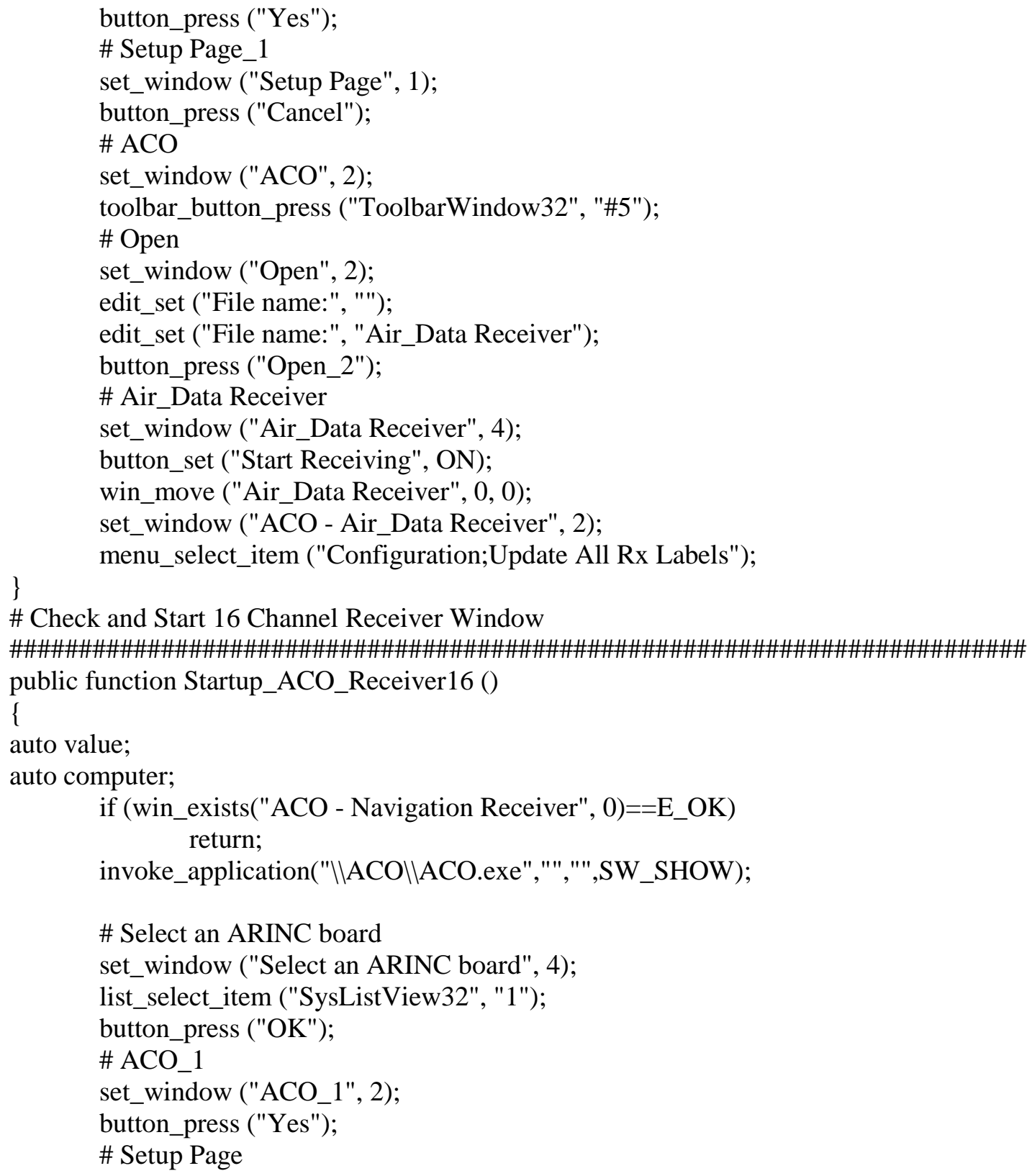


set_window ("Setup Page", 1);

button_press ("Cancel");

\# ACO

set_window ("ACO", 2);

toolbar_button_press ("ToolbarWindow32", "\#5");

\section{\# Open}

set_window ("Open", 2);

edit_set ("File name:", "");

edit_set ("File name:", "Navigation Receiver");

button_press ("Open_2");

\# Navigation Receiver

set_window ("Navigation Receiver", 4);

button_set ("Start Receiving", ON);

win_move ("Navigation Receiver", 0, 0);

set_window ("ACO - Navigation Receiver", 2);

menu_select_item ("Configuration;Update All Rx Labels");

\}

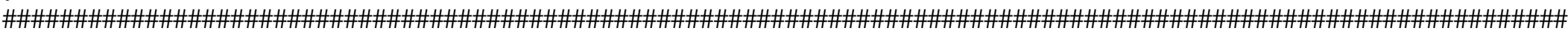

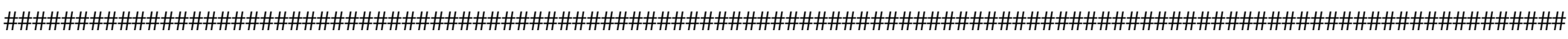
\#\#\#\#\#\#!!!USER FUNCTIONS!!!\#\#\#\#\#\#\#

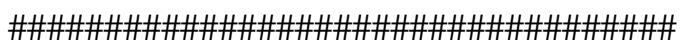

\# This function verifies that the correct SSM was attributed to the specified label.

\# Choose which function you want to test. "Air_Data" for Air Data, etc.

public function Check_Label_SSM (step, Function, Port, Label, Expected_SSM, Expected_SSM2, BITE)

\{

auto Actual_SSM;

auto rate;

auto ACO_window;

auto label_type;

auto Description;

auto i;

if (Function $==$ "Air_Data")

ACO_window = "Air_Data Receiver";

else

ACO_window = "Navigation Receiver";

set_window (ACO_window, 5); 


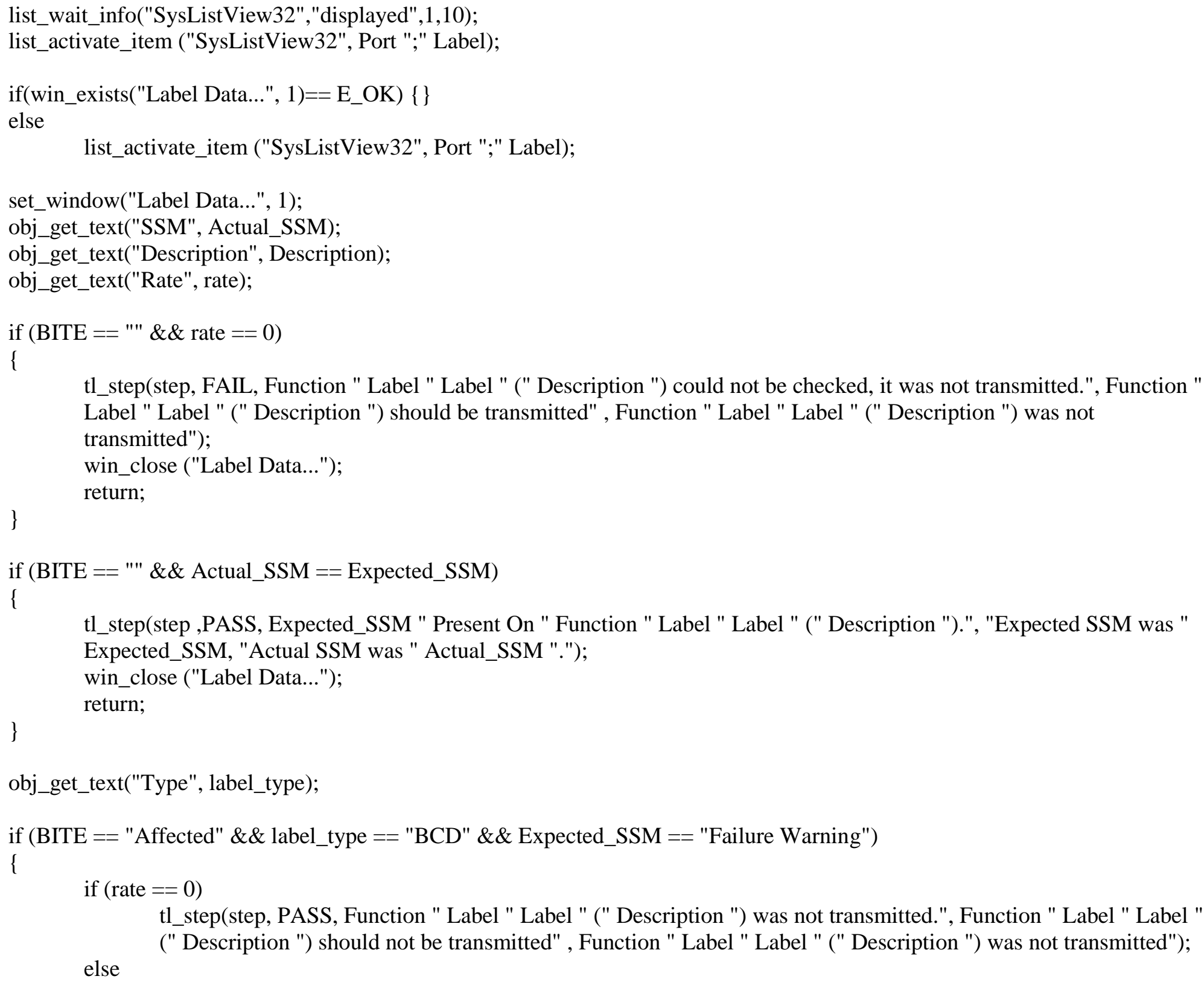

tl_step(step, PASS, Function " Label " Label " (" Description ") was not transmitted.", Function " Label " Label " else

(" Description ") should not be transmitted" , Function " Label " Label " (" Description ") was not transmitted"); 
tl_step(step, FAIL, Function " Label " Label " (" Description ") was transmitted.", Function " Label " Label " (" Description ") should not be transmitted" , Function " Label " Label " (" Description ") was transmitted");

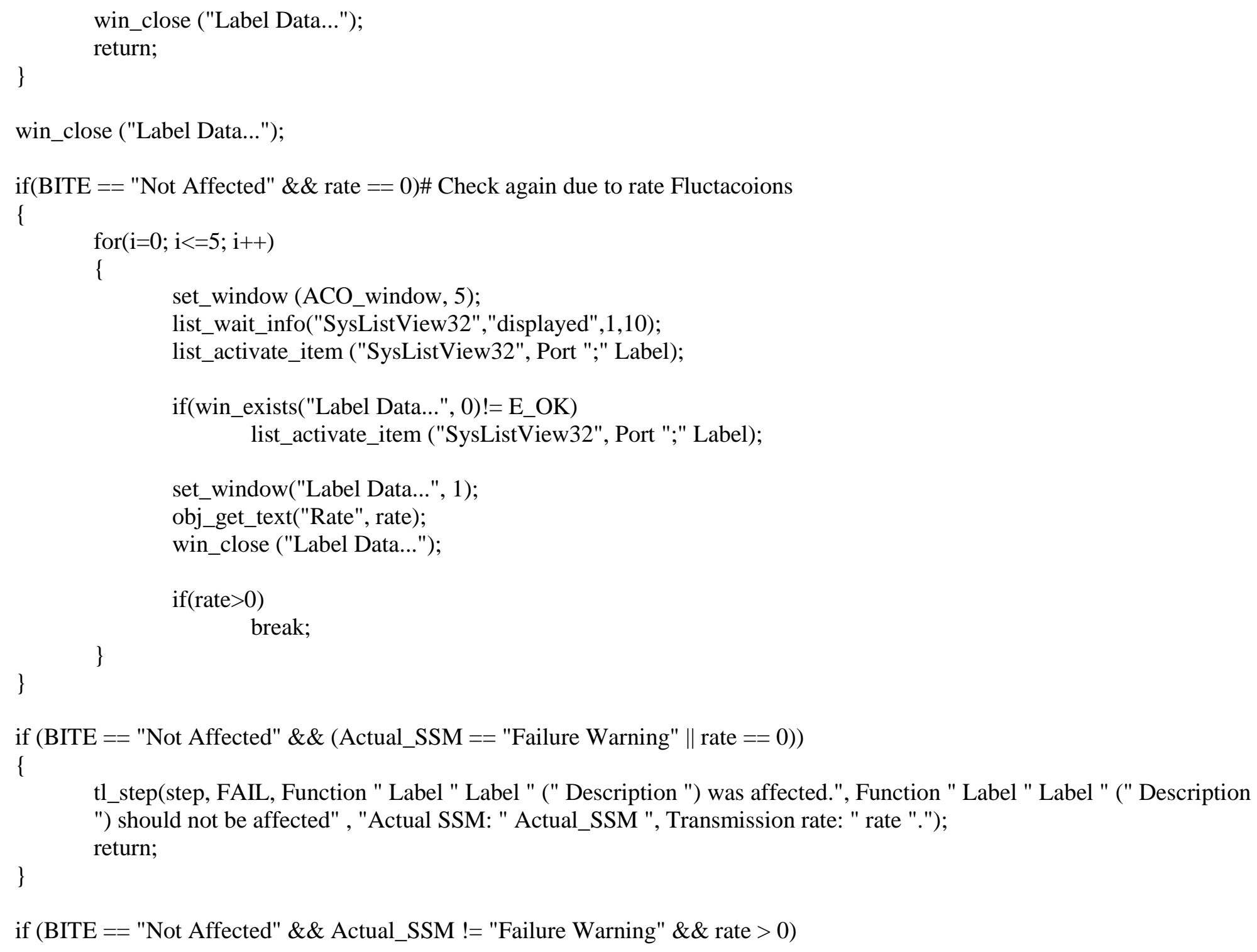




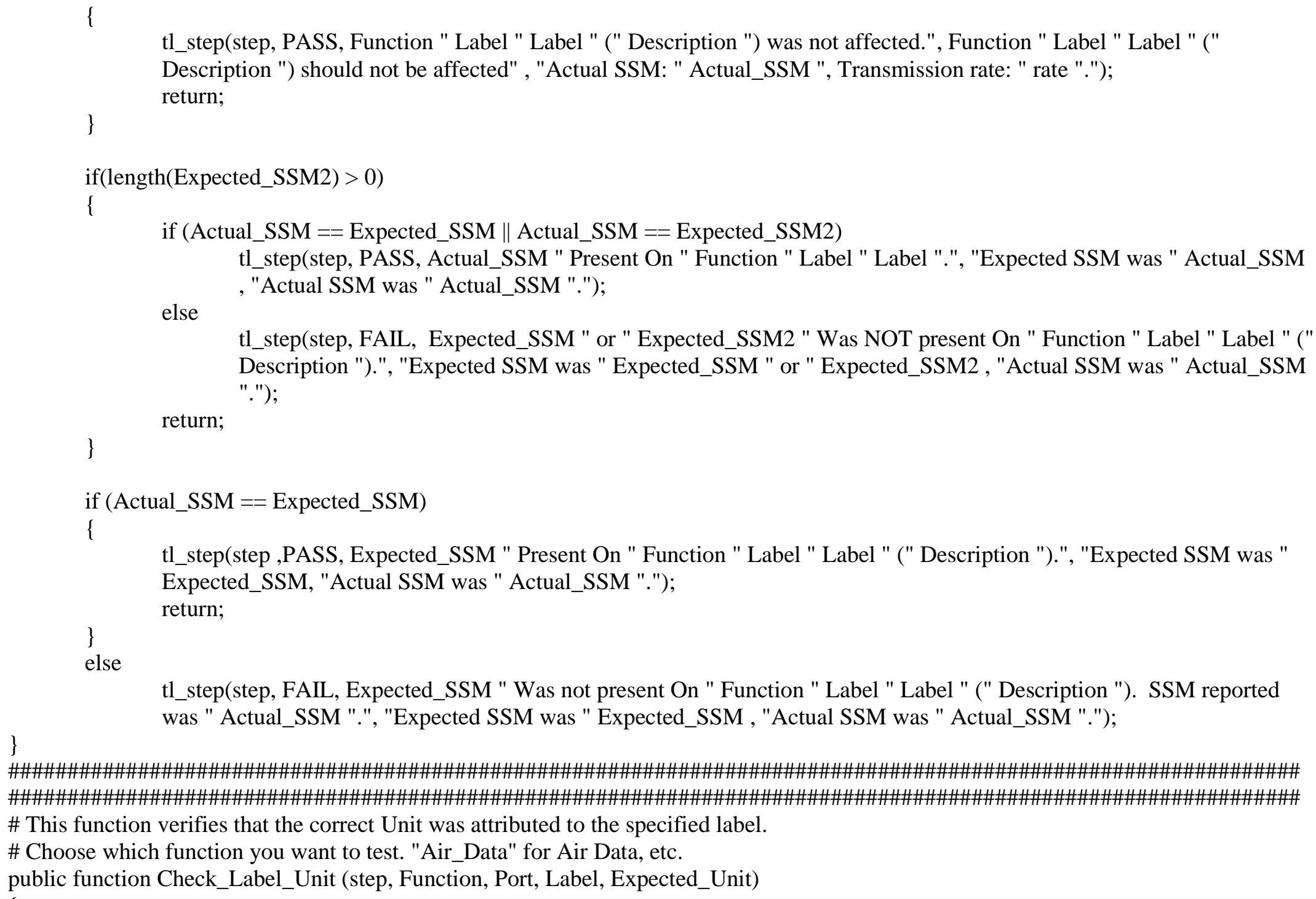


auto $\mathrm{ACO} \_$window;

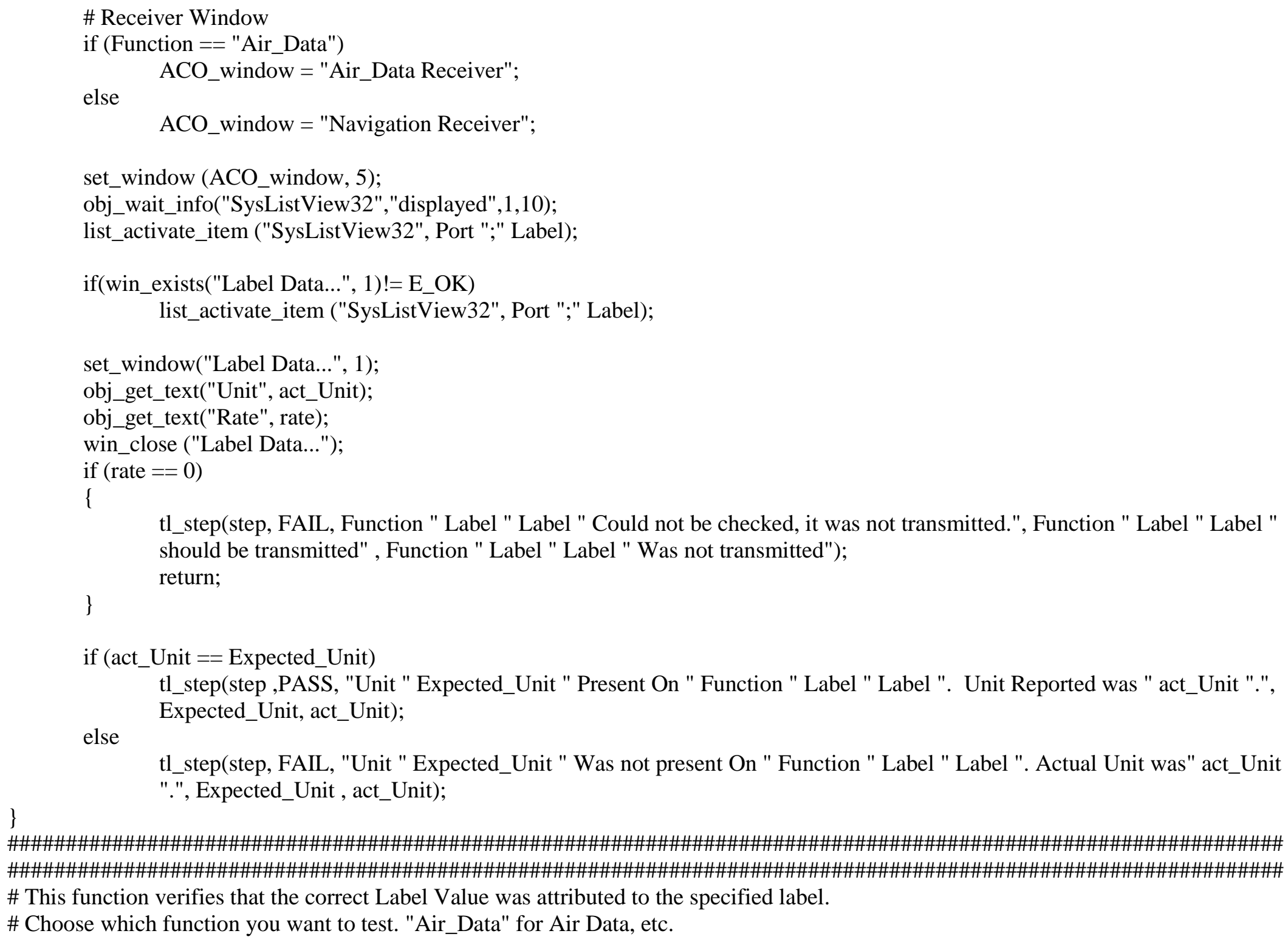


public function Check_Label_All_Ports (step, Function, Ports, Label, Min_Tolerance, Max_Tolerance)

\{

auto act_Value;

auto rate;

auto i;

auto ACO_window;

auto num_channels;

auto Channel[];

auto $\mathrm{n}$;

auto Type;

auto act_SSM;

auto new_SSM;

auto new_act_Value;

auto fail_flag;

\# Receiver Window

if (Function == "Air_Data")

else

ACO_window = "Air_Data Receiver";

ACO_window = "Navigation Receiver";

num_channels $=$ split(Ports, Channel, ",");

\#Get Baseline Label Value to compare to the other Ports

set_window (ACO_window, 5);

obj_wait_info("SysListView32","displayed",1,10);

list_activate_item ("SysListView32", Channel[1] ";" Label);

if(win_exists("Label Data...", 1)!= E_OK)

list_activate_item ("SysListView32", Channel[1] ";" Label);

set_window("Label Data...", 1);

obj_get_text("Value", act_Value);

obj_get_text("Rate", rate);

obj_get_text("SSM", act_SSM);

win_close ("Label Data...");

if (rate $==0$ ) 
tl_step(step, FAIL, Function " Label " Label " on Port " Channel[1] " Could not be checked, it was not transmitted.", Function " Label " Label " should be transmitted", Function " Label " Label " Was not transmitted");

return;

\}

fail_flag = 0;

\#Compare baseline Label Value with other Port

for $(\mathrm{n}=2 ; \mathrm{n}<=$ num_channels; $\mathrm{n}++)$

\{

set_window (ACO_window, 5);

obj_wait_info("SysListView32","displayed",1,10);

list_activate_item ("SysListView32", Channel[n] ";" Label);

if(win_exists("Label Data...", 1)!= E_OK)

list_activate_item ("SysListView32", Channel[n] ";" Label);

set window("Label Data...", 1);

obj_get_text("Value", new_act_Value);

obj_get_text("Rate", rate);

obj_get_text("Type", Type);

obj_get_text("SSM", new_SSM);

win_close ("Label Data...");

if $($ rate $==0)$

tl_step(step, FAIL, Function " Label " Label " on Port " Channel[n] " Could not be checked, it was not transmitted.", Function " Label " Label " should be transmitted" , Function " Label " Label " Was not transmitted");

return;

\}

if $($ Type $==$ "BCD")

if(new_SSM $==$ act_SSM $\& \&$ new_act_Value $>=$ act_Value - Min_Tolerance $\& \&$ new_act Value $<=$ act Value + Max_Tolerance)

continue;

else 
tl_step(step, FAIL, "The expected BCD value (or its SSM) of " act_Value " was NOT present or Out-ofTolerance on " Function " Label " Label " on Port " Channel[n] ". Actual Value and its SSM were " new_act_Value "and" new_SSM ".", act_Value " with tolerance: between " act_Value - Min_Tolerance " and " act_Value + Max_Tolerance " and SSM of " act_SSM, new_act_Value "and SSM of " new_SSM); fail_flag = 1;

\} continue;

\}

if (Type $==$ "SPL")

\{

if (new_act_Value == act_Value)

continue;

else

\{

tl_step(step, FAIL, "The expected discrete value of " act_Value " was NOT present on " Function " Label " Label " on Port " Channel[n]". Actual Value was " new_act_Value ".", act_Value, new_act_Value); fail_flag = 1;

continue;

\}

if (new_act_Value $>=$ act_Value - Min_Tolerance $\& \&$ new_act_Value $<=$ act_Value + Max_Tolerance) continue;

else

\{

tl_step(step, FAIL, "The expected value of " act_Value " was NOT present (or within tolerance) on " Function " Label " Label " on Port " Channel[n] ". Actual Value was " new_act_Value ".", act_Value " with tolerance: between " act_Value - Min_Tolerance " and " act_Value + Max_Tolerance, new_act_Value);

\} fail_flag = 1;

\}

if (fail_flag ==0)

tl_step(step, PASS, "The values on " Function " Label " Label " on Ports " Ports " all match the value of " act_Value ".", "All Label values should match on each port.", "The label values match.");

else 
tl_step(step, FAIL, "The values on " Function " Label " Label " on Ports " Ports " do NOT all match the value of " act_Value ".", "All Label values should match on each port.", "The label values did NOT all match.");

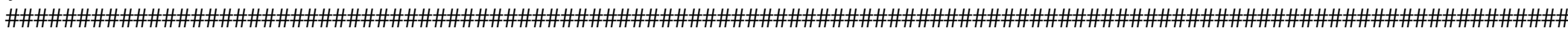

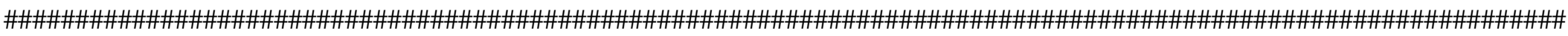
\# This function verifies that the correct Label Value was attributed to the specified label.

\# Choose which function you want to test. "Air_Data" for Air Data, etc.

public function Check_Label_Value_With_Mask (step, Function, Port, Label, Expected_Value, Mask)

\{

auto act_Value;

auto rate;

auto ReturnValue;

auto i;

auto ACO_window;

auto func[];

split(Function, func, " "); \# Second element denotes possible Test type (EMI, ATP, etc)

\# Receiver Window

if (func[1] == "Air_Data")

ACO_window = "Air_Data Receiver";

else

ACO_window = "Navigation Receiver";

if (func[2] == "ATP")

\{

if (func[1] == "Air_Data")

ACO_window $=$ "Air_Data HW RX";

\}

ACO_window = "GPS_Navigation HW RX";

if (func[2] == "EMI")

\{

if (func[1] == "Air_Data")

ACO_window = "EMI Air_Data RX";

else 


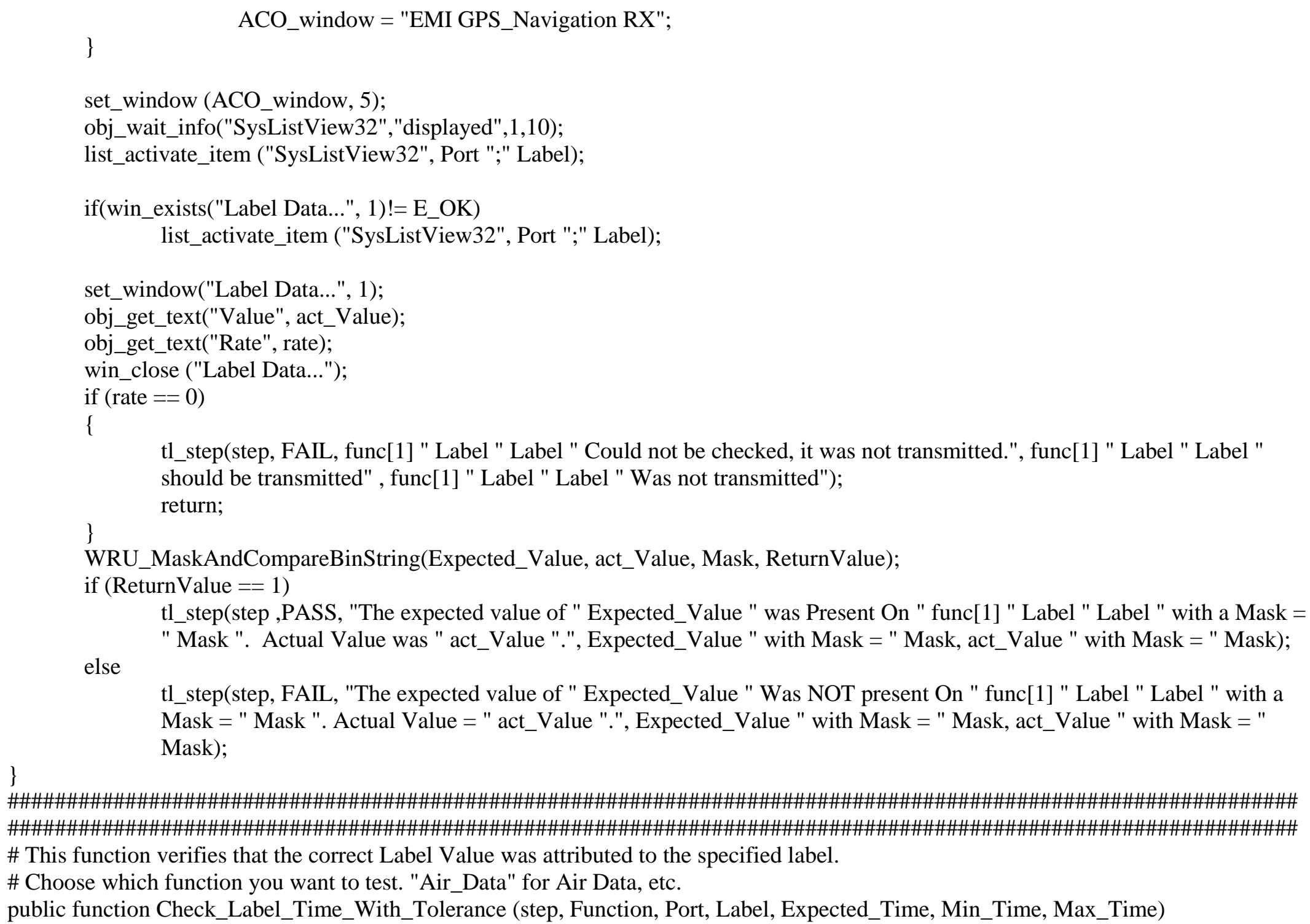


auto Min_Time1;

auto Max_Time1;

auto rate;

auto Type;

auto SSM;

auto i;

auto ACO_window;

\# Receiver Window

if (Function == "Air_Data")

else

$$
\text { ACO_window = "Air_Data Receiver"; }
$$

ACO_window = "Navigation Receiver";

set_window (ACO_window, 5);

obj_wait_info("SysListView32","displayed",1,10);

list_activate_item ("SysListView32", Port ";" Label);

if(win_exists("Label Data...", 1)!= E_OK)

list_activate_item ("SysListView32", Port ";" Label);

set_window("Label Data...", 1);

obj_get_text("Value", act_Value);

obj_get_text("Rate", rate);

obj_get_text("Type", Type);

obj_get_text("SSM", SSM);

win_close ("Label Data...");

if $($ rate $==0)$

tl_step(step, FAIL, Function " Label " Label " Could not be checked, it was not transmitted.", Function " Label " Label " should be transmitted" , Function " Label " Label " Was not transmitted"); return;

\}

act_Value1 = repl_str(act_Value, ":", "");

Min_Time1 = repl_str(Min_Time, ":", "");

Max_Time1 = repl_str(Max_Time, ":", ""); 


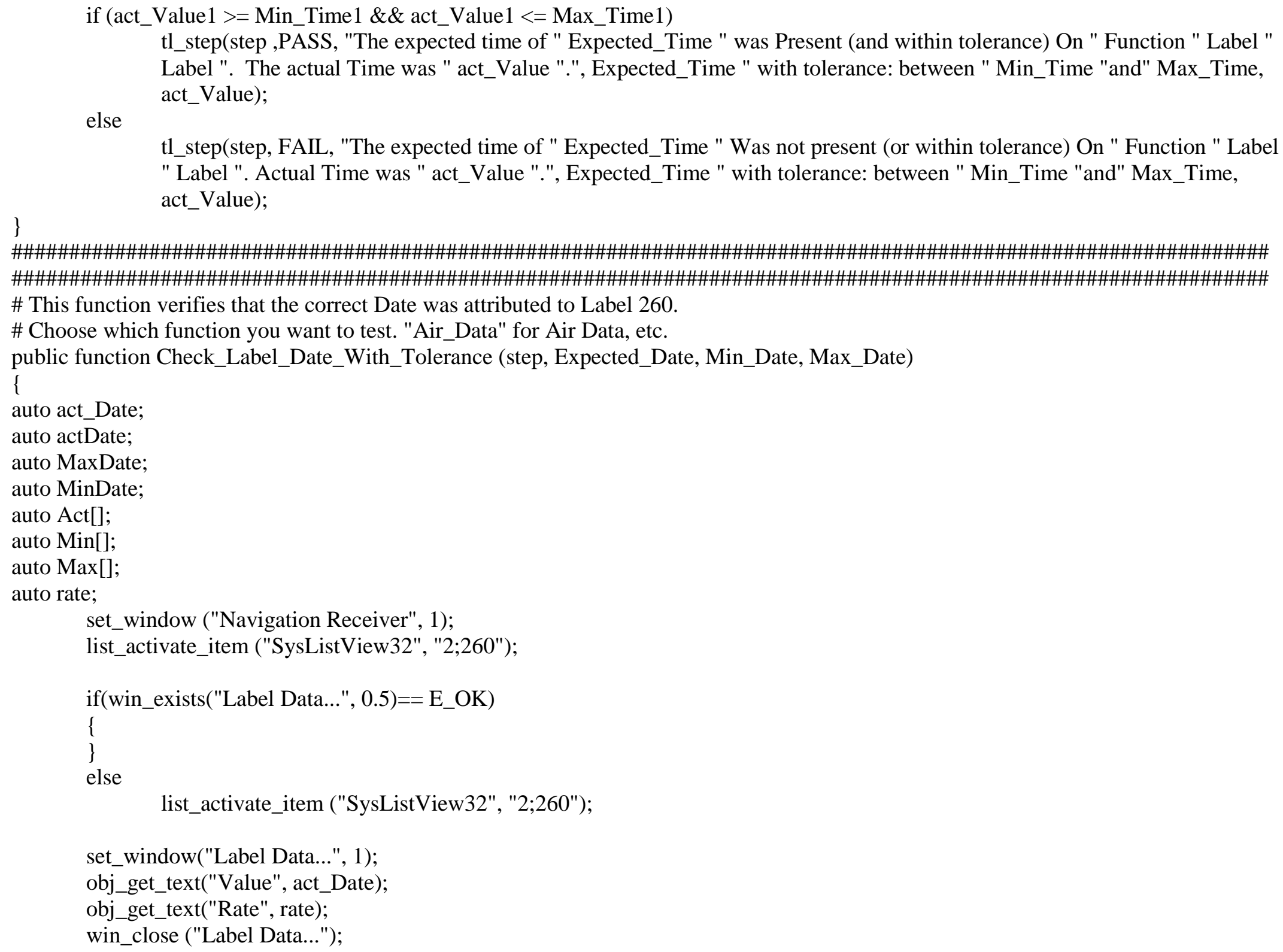




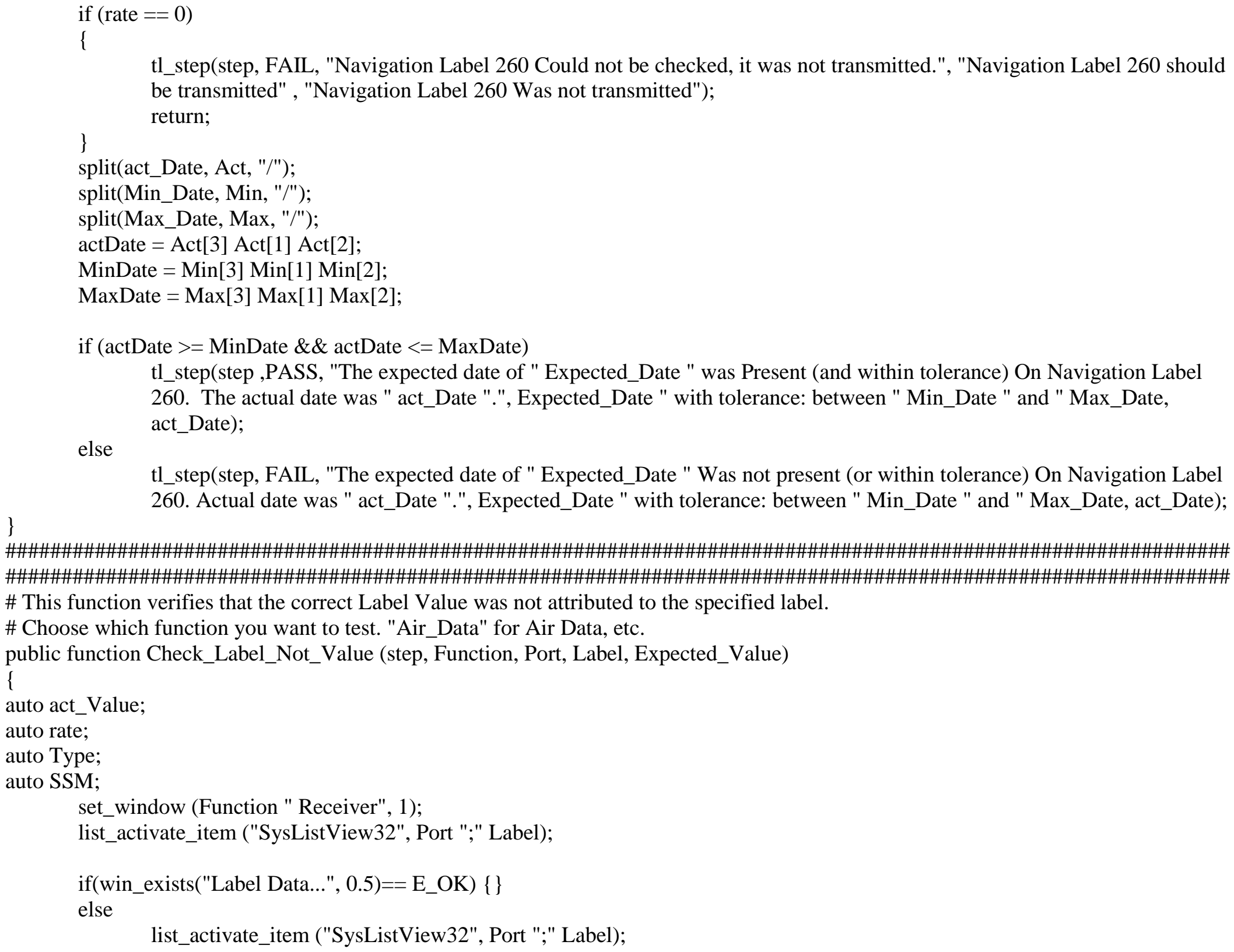




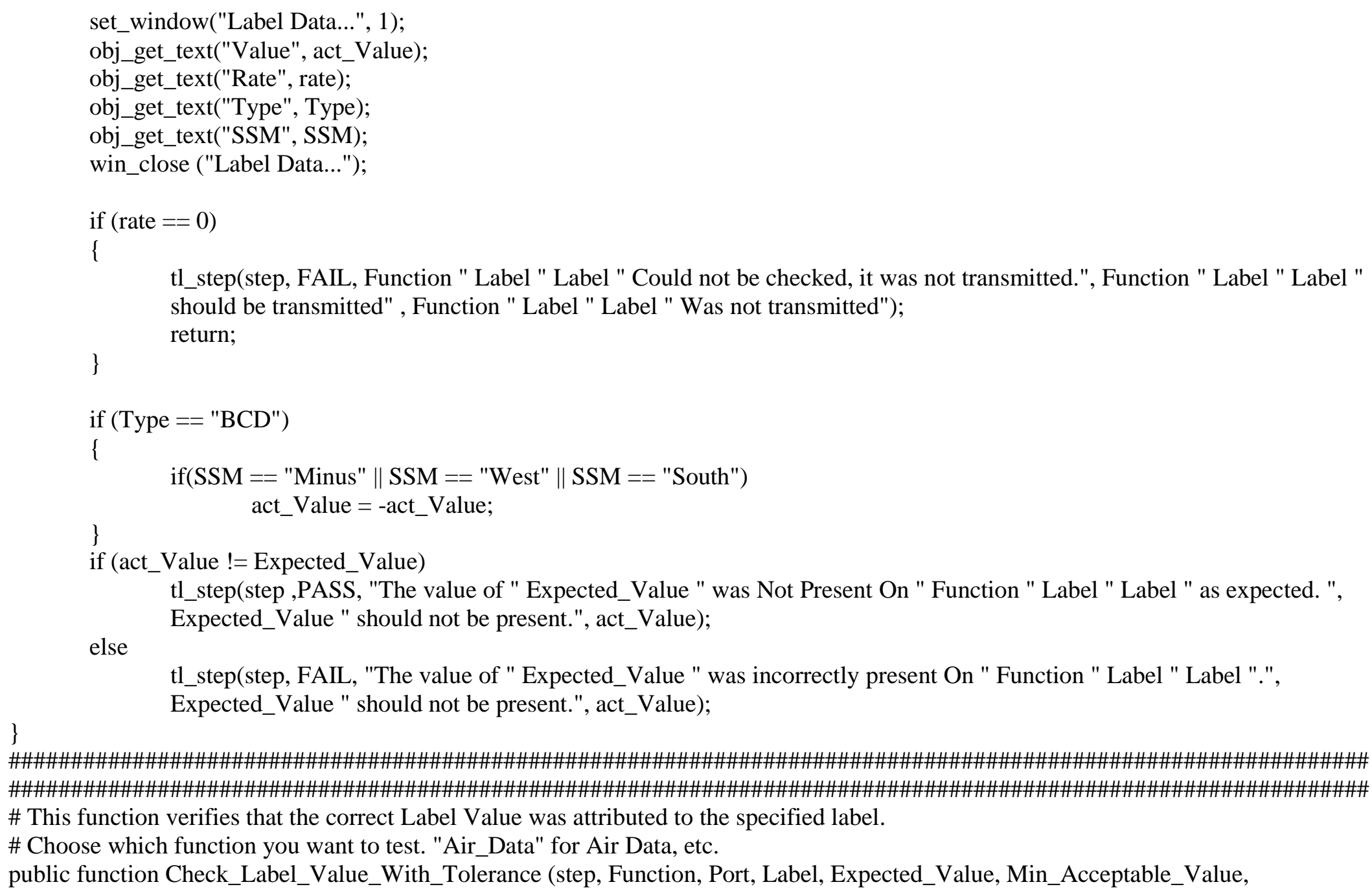


auto SSM;

auto i;

set_window (Function " Receiver", 1);

list_activate_item ("SysListView32", Port ";" Label);

if(win_exists("Label Data...", 0.5)==E_OK) \{\}

else

list_activate_item ("SysListView32", Port ";" Label);

set_window("Label Data...", 1);

obj_get_text("Value", act_Value);

obj_get_text("Rate", rate);

obj_get_text("Type", Type);

obj_get_text("SSM", SSM);

win_close ("Label Data...");

if $($ rate $==0)$

tl_step(step, FAIL, Function " Label " Label " Could not be checked, it was not transmitted.", Function " Label " Label " should be transmitted" , Function " Label " Label " Was not transmitted"); return;

\}

if $($ Type $==$ "BCD")

\{

if(SSM == "Minus" || SSM == "West" || SSM == "South")

act_Value $=$-act_Value;

\}

if (act_Value $>=$ Min_Acceptable_Value \&\& act_Value $<=$ Max_Acceptable_Value)

tl_step(step ,PASS, "The expected value of " Expected_Value " was Present (and within tolerance) On " Function " Label

" Label ". The actual value was " act_Value ".", Expected_Value " with tolerance: between " Min_Acceptable_Value " and "Max_Acceptable_Value, act_Value);

else

tl_step(step, FAIL, "The expected value of " Expected_Value " Was not present (or within tolerance) On " Function "

Label " Label ". Actual Value was " act_Value ".", Expected_Value " with tolerance: between " Min_Acceptable_Value " and "Max_Acceptable_Value, act_Value); 


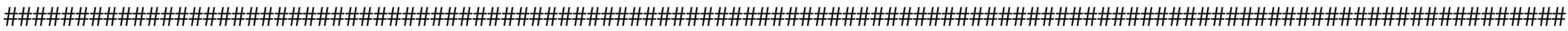

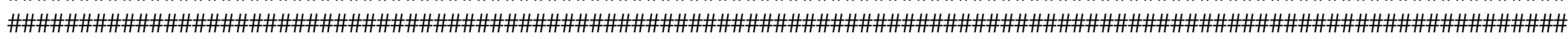
\# This function verifies that the Label was either Transmitted or Not Transmitted per User Input.

\# Choose which function you want to test. "Air_Data" for Air Data, etc.

public function Check_Label_Rate (step, Function, Port, Label, Expected_Rate)

auto rate;

auto i;

auto ACO_window;

\# Receiver Window

if (Function $==$ "Air_Data")

else

$$
\text { ACO_window = "Air_Data Receiver"; }
$$

ACO_window = "Navigation Receiver";

set_window (ACO_window, 5);

obj_wait_info("SysListView32","displayed",1,10);

list_activate_item ("SysListView32", Port ";" Label);

if(win_exists("Label Data...", 1)!= E_OK)

list_activate_item ("SysListView32", Port ";" Label);

set_window("Label Data...", 1);

obj_get_text("Rate", rate);

win_close ("Label Data...");

if $($ rate $==0 \& \&$ Expected_Rate $!=0$ )

\{

tl_step(step, FAIL, Function " Label " Label " Could not be checked, it was not transmitted.", Function " Label " Label " should be transmitted" , Function " Label " Label " Was not transmitted");

return;

\}

if $($ rate $>=$ Expected_Rate-(Expected_Rate $* 0.1) \& \&$ rate $<=$ Expected_Rate+(Expected_Rate $* 0.1)$ )

tl_step(step, PASS, Function " Label " Label " Was Transmitted at correct Rate of " Expected_Rate " Hz. The actual rate was " rate ".", Function " Label " Label " should be transmitted at " Expected_Rate " Hz." , Function " Label " Label "

Was Transmitted at " rate " Hz.");

else 
tl_step(step, FAIL, Function " Label " Label " Was NOT Transmitted at correct Rate of " Expected_Rate " Hz. Actual Rate was " rate " Hz.", Function " Label " Label " should be transmitted at " Expected_Rate " Hz." , Function " Label "

\}

Label " Was Transmitted at " rate " Hz.");

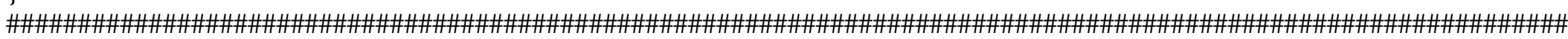

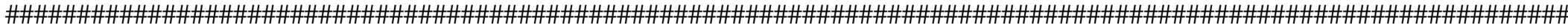
\# This function verifies that the Label was either Transmitted or Not Transmitted per User Input.

\# Choose which function you want to test. "Air_Data" for Air Data, etc.

public function Check_Label_Transmission (step, Function, Port, Label, Expected_Transmission)

\{

auto rate;

auto i;

auto ACO_window;

\# Receiver Window

if (Function $==$ "Air_Data")

if (Function == "Navigation")

ACO_window = "Air_Data Receiver";

ACO_window = "Navigation Receiver";

if (Function $==$ "ATP Air_Data")

ACO_window = "Air_Data HW RX";

if (Function $==$ "ATP GPS_Navigation")

ACO_window = "GPS_Navigation HW RX";

set_window (ACO_window, 5);

obj_wait_info("SysListView32","displayed",1,10);

list_activate_item ("SysListView32", Port ";" Label);

if(win_exists("Label Data...", 1)!=E_OK)

list_activate_item ("SysListView32", Port ";" Label);

set_window("Label Data...", 1);

obj_get_text("Rate", rate);

win_close ("Label Data...");

if(Expected_Transmission=="Not Transmitted")

\{ 


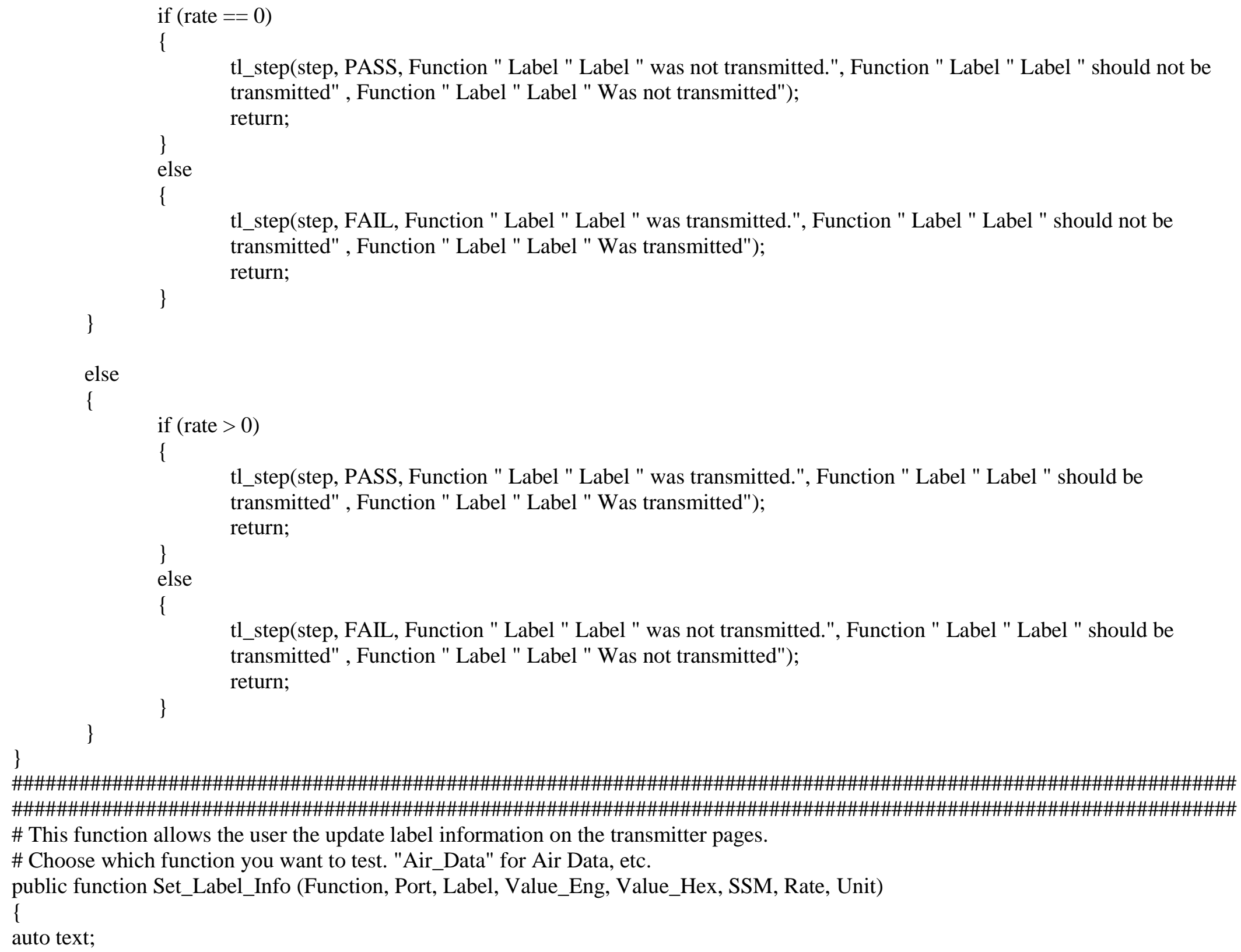


auto ACO_window;

\# Receiver Window

if (Function $==$ "Air_Data")

ACO_window = "Air_Data Unit " Unit;

else

ACO_window = "Test_Station Transmitter";

win_activate(ACO_window);

set_window (ACO_window, 5);

obj_wait_info("SysListView32","displayed",1,10);

obj_mouse_click("SysListView32",1,1,LEFT);

list_select_item ("SysListView32", Port ";" Label);

obj_get_text("Description", text);

if(length(Value_Eng) $>0)$

\{

button_press ("Eng_0");

\# 42 - Set Longitude

set_window (Label " - " text, 11);

if(length(Value_Eng) $>0$ )

edit_set ("Value (Eng)_0", Value_Eng);

if $($ length $(\mathrm{SSM})>0$ )

list_select_item ("Value (Eng)_1", SSM);

if(length(Unit) $>0$ )

list_select_item ("ComboBox", Unit); \# Item Number 1;

button_press ("OK");

set_window (ACO_window, 5);

if (length $($ Rate $)>0$ )

edit_set ("Rate (Hz)", Rate);

button_press ("Update");

\} 


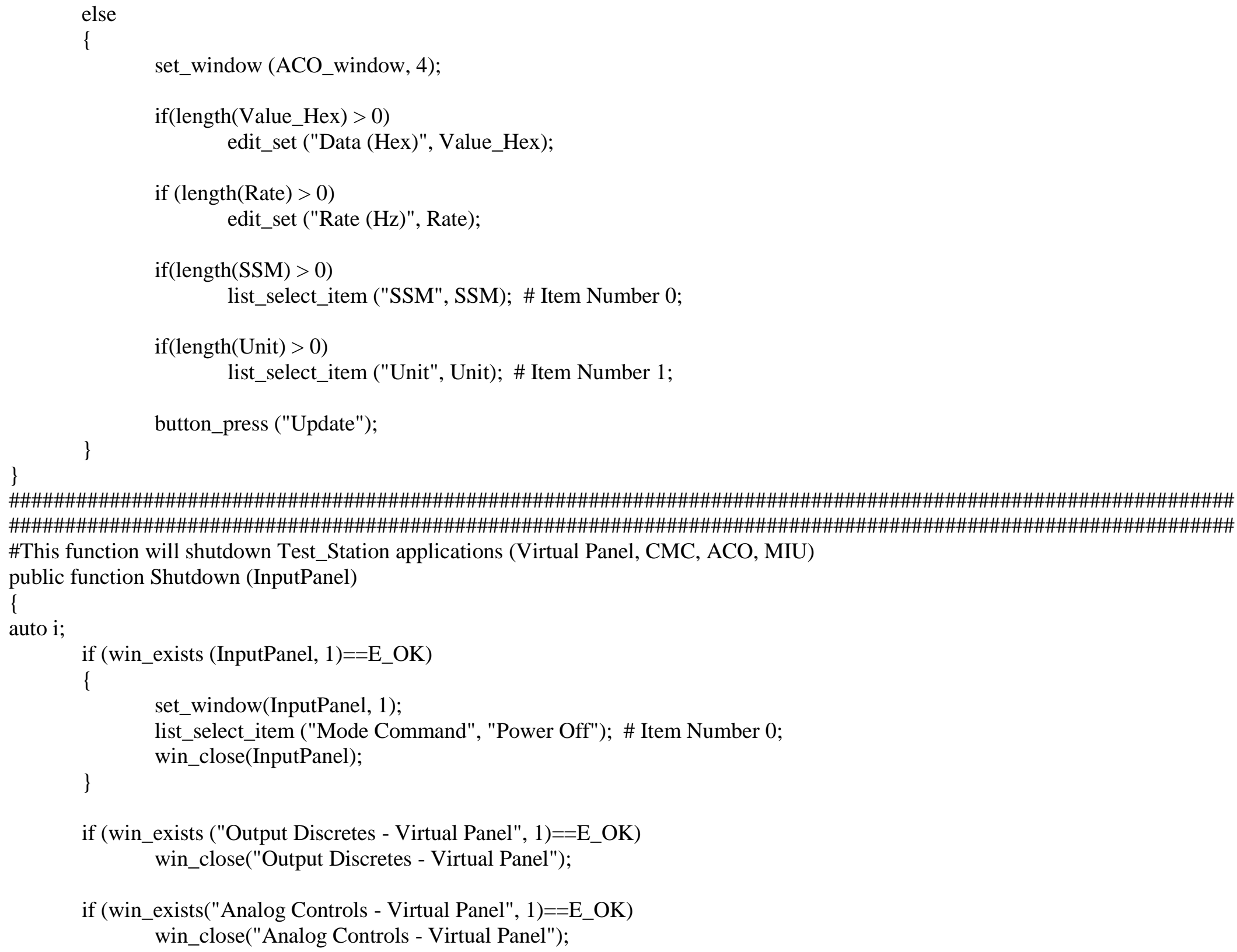


if (win_exists("Power Controls - Virtual Panel", 1)==E_OK) win_close("Power Controls - Virtual Panel");

if (win_exists("ACO - Test_Station Transmitter", 1)==E_OK) win_close("ACO - Test_Station Transmitter");

if (win_exists("ACO - Navigation Receiver", 1)==E_OK) win_close("ACO - Navigation Receiver");

if (win_exists("ACO - Navigation Receiver", 1)==E_OK) win_close("ACO - Navigation Receiver");

if (win_exists("ACO - Air_Data Receiver", 1)==E_OK) win_close("ACO - Air_Data Receiver");

if (win_exists("ACO - Air_Data Receiver", 1)==E_OK) win_close("ACO - Air_Data Receiver");

if (win_exists("MIU", 1)==E_OK) win_close("MIU");

if(win_exists("ACO - Air_Data HW TX",1)==E_OK) win_close("ACO - Air_Data HW TX");

if (win_exists("ACO - Air_Data HW RX",1)==E_OK) win_close("ACO - Air_Data HW RX");

if(win exists("ACO - GPS Navigation HW TX",1)==E OK) win_close("ACO - GPS_Navigation HW TX");

if(win_exists("ACO - GPS_Navigation HW RX",1)==E_OK) win_close("ACO - GPS_Navigation HW RX");

if(win_exists("Air_Data Unit 1", 1)==E_OK)

set_window ("Air_Data Unit 1", 1);

button_set ("Start Transmitting", OFF);

win_close("Air_Data Unit 1");

set_window ("Air_Data Unit 2", 1);

button_set ("Start Transmitting", OFF);

win_close("Air_Data Unit 2");

set_window ("Air_Data Unit 3", 1);

button_set ("Start Transmitting", OFF);

win_close("Air_Data Unit 3");

set_window("ACO",1);

win_close("ACO"); 


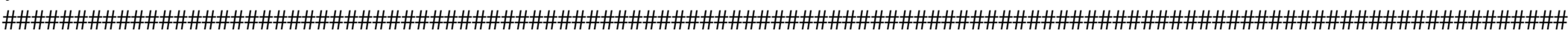

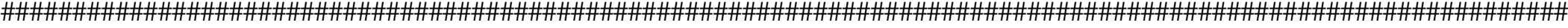
public function Create_Data_Table(Table_Name,Folder_Path, num_column, Column_Names)

auto Table_Path;

auto column[];

auto i;

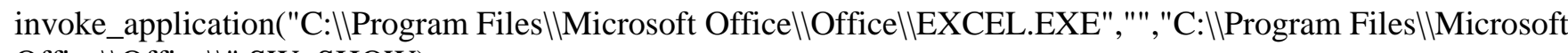
OfficellOfficell",SW_SHOW);

Table_Path = Folder_Path \& Table_Name;

Column_Names = repl_str(Column_Names, " ", "");

split(Column_Names, column, ",");

\# Microsoft Excel - Blank

set_window ("Microsoft Excel - Book1", 5);

obj_mouse_click ("Book1", 42, 28, LEFT);

for $(\mathrm{i}=1 ; \mathrm{i}<=100$; $\mathrm{i}++)$

\{

if(column[i])

\{

obj_mouse_dbl_click ("EXCEL<", 118, 9, LEFT);

obj_type ("EXCEL<",column[i]);

obj_type ("EXCEL<","<kTab>");

else

break;

\# Microsoft Excel - Figure

win_close ("Microsoft Excel - Book1");

\# Microsoft Excel

set_window ("Microsoft Excel", 2);

button_press ("Yes")

\# Save As

set_window ("Save As", 2);

obj_type ("RichEdit20W", Table_Path); 


\section{obj_type ("RichEdit20W","<kReturn>");}

if(win_exists("Microsoft Excel", 1)==E_OK)

\{

set_window ("Microsoft Excel", 3);

\}

button_press ("Yes");

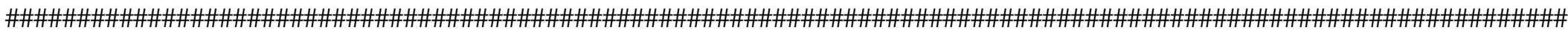

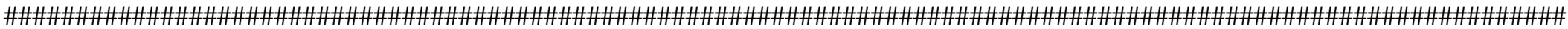

\# This function allows a user to add labels in the standard Air_Data or Navigation ACO Pages

public function Add_Label(Designation, Port, Label)

auto Function;

auto ACO_Window;

split(Designation, array, " ");

Function = array[1];

if(Function == "Air_Data")

ACO_Window = "Air_Data Receiver";

else

ACO_Window = "Navigation Receiver";

set_window (ACO_Window, 4);

list_select_item ("Port", Port); \# Item Number 0;

button_set ("By Label \#", ON);

list_select_item ("ComboBox", Label); \# Item Number 82;

button_press ("Lookup");

if(win_exists("Select a Function》ISubFunction Pair", 1)==E_OK)

\{

set_window ("Select a Function ISSubFunction Pair", 4);

button_press ("OK");

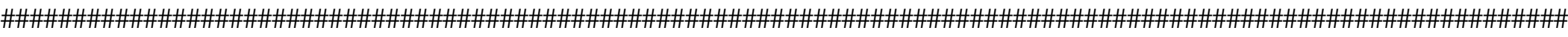

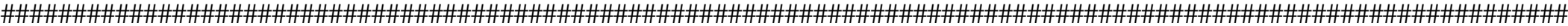

\# This function allows a user to create new labels in the standard Air_Data or Navigation ACO Pages 
public function Create_Label(Designation, Port, Label, Name, Partition, SubFunction, Units, Value_Hex, Rate, Unit) \{ auto Transmitter; auto Receiver; auto Function; auto array[];

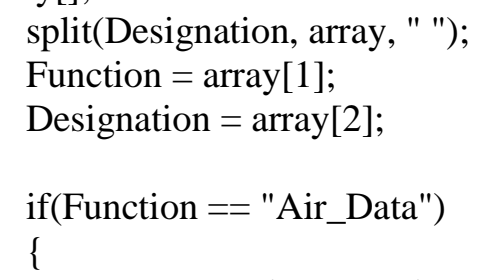




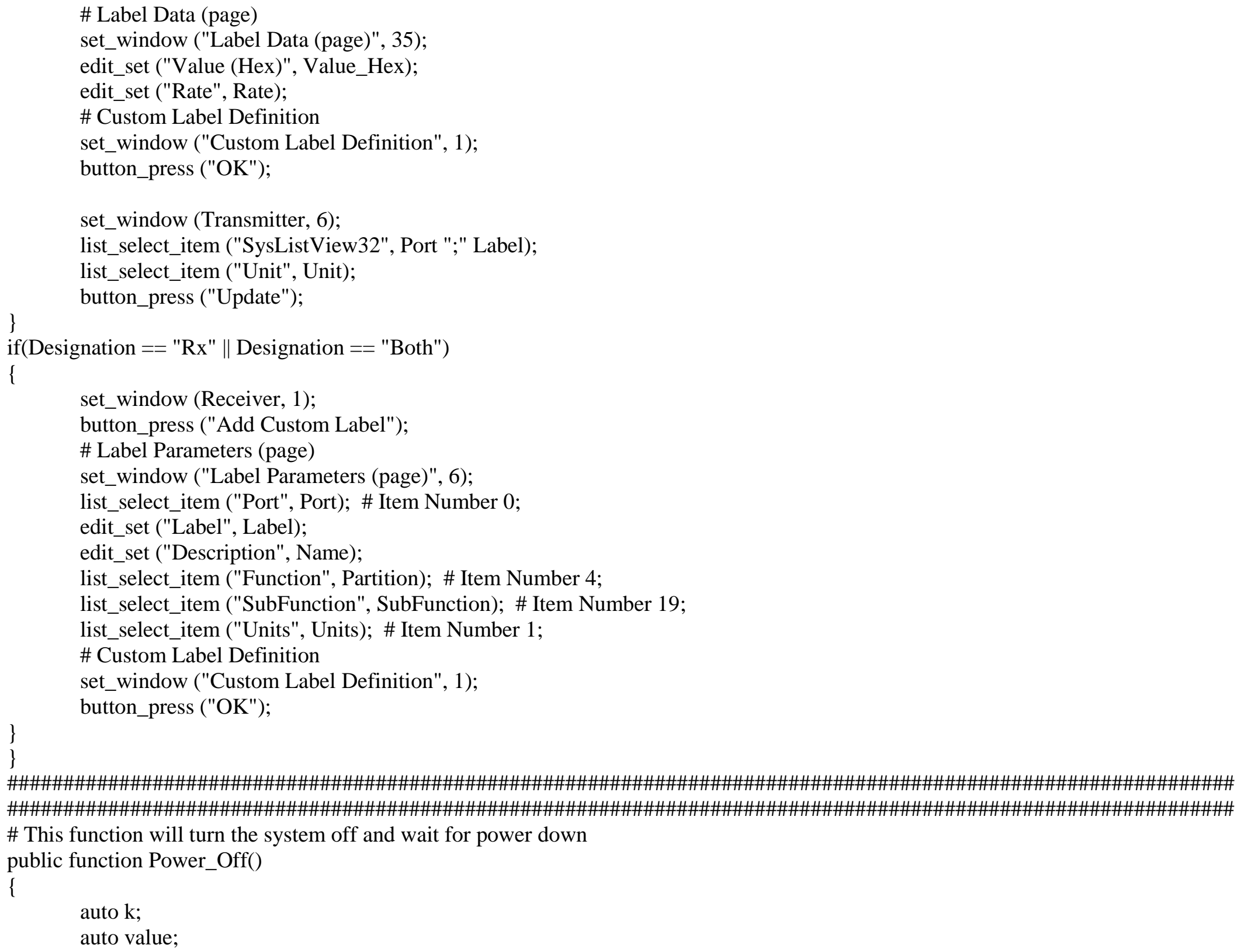


auto start_time;

set_window ("Input Discretes - System3 - Virtual Panel", 1);

list_select_item ("Mode Command", "Power Off"); \# Set M1 Off

start_time = get_time () ;

for $(\mathrm{k}=0 ; \mathrm{k}++)$

if (get_time() - start_time > 15) \# Wait 15 seconds max

set_window ("Input Discretes - System3 - Virtual Panel", 5);

list_select_item ("Mode Command", "Power Off"); \# Try turning off again

break;

set_window("Output Discretes - Virtual Panel", 1);

obj_get_info("System On ODSC","enabled",value);

if $($ value $==0$ )

\{

\}

break;

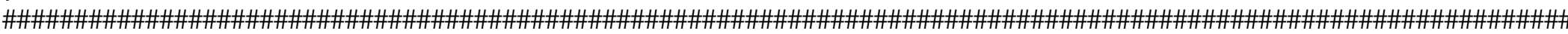

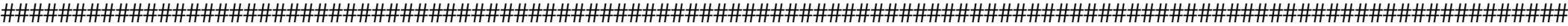
\# Shut down MIU

public function MIU_Shutdown()

set_window ("MIU", 5);

toolbar_button_press ("ToolbarWindow32", "\#9");

\}

win_close ("MIU");

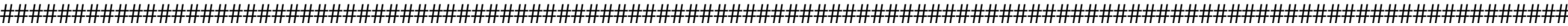

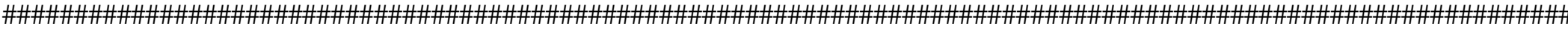
\#This function will round a number to the desired decimal place public function Round (Number, Decimal_Places, out Rounded_Number)

auto Fractional; 
auto Scale;

auto Scaled_Number;

auto ReverseSign;

ReverseSign $=0$;

Scale $=10^{\wedge}$ Decimal_Places;

Scaled_Number $=$ Number $*$ Scale;

Rounded_Number $=$ int $($ Scaled_Number $)$;

Fractional $=($ Scaled_Number $)-$ Rounded_Number;

if(Number $<=0.0)$

\{

Number $=-$ Number;

ReverseSign $=1$

if $($ Fractional $>=0.5)$

\{

\}

Rounded_Number $=($ Rounded_Number +1$) /($ Scale $)$;

else

\{

\}

Rounded_Number $=$ Rounded_Number/(Scale);

if $($ ReverseSign $==1)\{$ Number $=-$ Number; $\}$

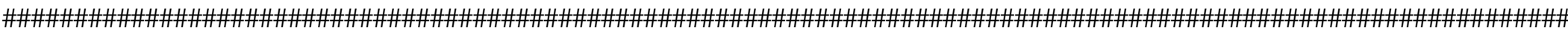

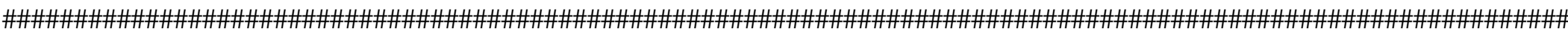

\#This function will convert a Hex string to an integer

public function StrToInt (strIn, out intOut)

auto Hex;

auto Digit;

auto i;

for $(\mathrm{i}=1 ; \mathrm{i}<$ length $(\operatorname{strIn})+1 ; \mathrm{i}++)$

Hex = substr(strIn,length(strIn)-i+1,1);

switch (Hex)

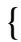




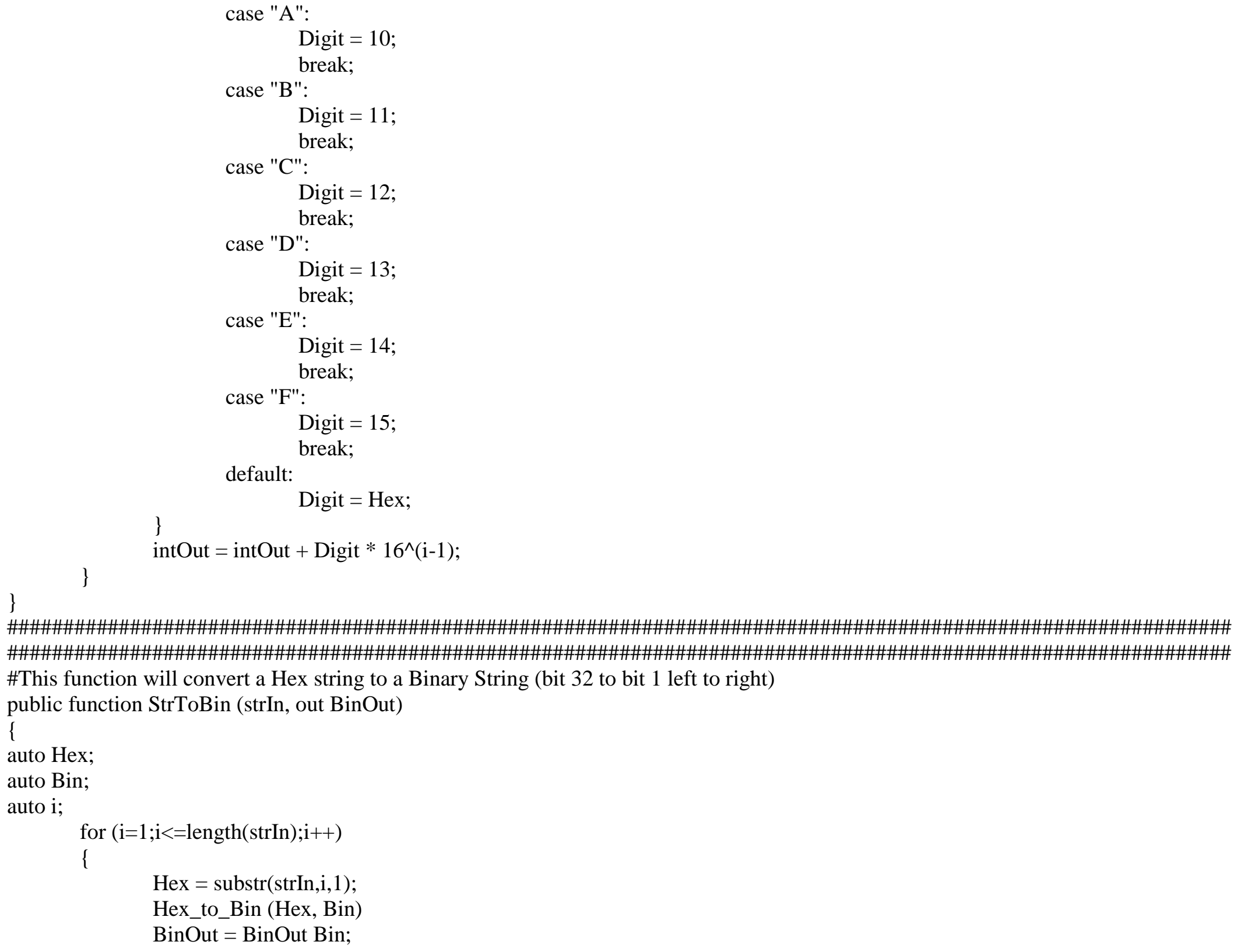


\}

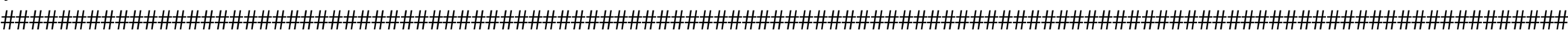

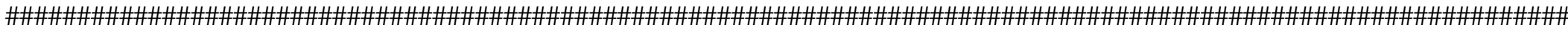
\#This function will convert a Bin string to a Decimal Value (msb to lsb is binary digits left to right)

public function BinToDec (strIn, out DecOut)

auto Dec;

auto Bin;

auto i;

for $(\mathrm{i}=0 ; \mathrm{i}<$ length(strIn);i++)

\{

Bin = substr(strIn,length(strIn)-i,1);

\}

DecOut $=$ DecOut + Bin* $\left(2^{\wedge} \mathrm{i}\right)$

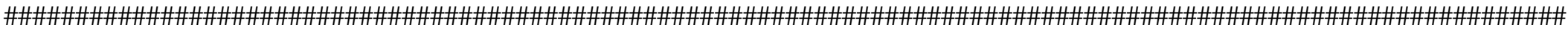

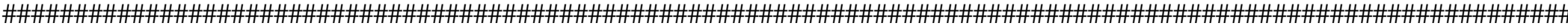

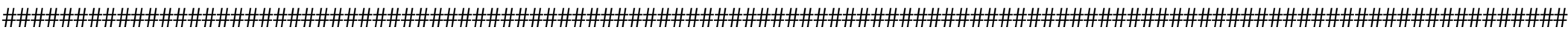
\#\#\#!!!STATIC FUNCTIONS!!!\#\#\#\#

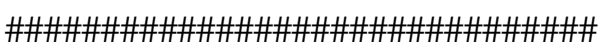
public function Hex_to_Bin (Hex, out Bin)

\{

$$
\begin{gathered}
\text { if(Hex == "0") } \\
\text { Bin = "0000"; } \\
\text { if(Hex == "1") } \\
\text { Bin = "0001"; } \\
\text { if(Hex == "2") } \\
\text { Bin = "0010"; } \\
\text { if(Hex == "3") } \\
\text { Bin = "0011"; } \\
\text { if(Hex == "4") } \\
\text { Bin = "0100"; } \\
\text { if(Hex == "5") } \\
\text { Bin = "0101"; } \\
\text { if(Hex == "6") } \\
\text { Bin = "0110"; }
\end{gathered}
$$




$$
\begin{aligned}
& \text { if(Hex == "7") } \\
& \text { Bin = "0111"; } \\
& \text { if(Hex == "8") } \\
& \text { Bin = "1000"; } \\
& \text { if(Hex == "9") } \\
& \text { Bin = "1001"; } \\
& \text { if(Hex == "A" }|| \text { Hex == "a") } \\
& \text { Bin = "1010"; } \\
& \text { if(Hex == "B" \| Hex == "b") } \\
& \text { Bin = "1011"; } \\
& \text { if(Hex == "C" } \| \text { Hex == "c") } \\
& \text { Bin = "1100"; } \\
& \text { if(Hex == "D" \| Hex == "d") } \\
& \text { Bin = "1011"; } \\
& \text { if(Hex == "E" } \| \text { Hex == "e") } \\
& \text { Bin = "1110"; } \\
& \text { if(Hex == "F" || Hex == "f") } \\
& \text { Bin = "1111"; }
\end{aligned}
$$

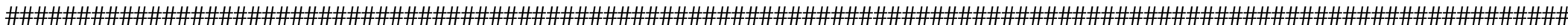

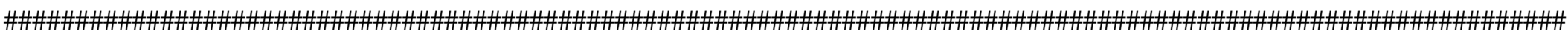
\# This function converts a bit into its place in a 4 bit hex word \# Ex: bit $3=0000000000001000=0008$

static function Bit16_to_Hex4 (Bit, out Hex)

\{

$$
\text { switch (Bit) }
$$

$$
\text { \{ }
$$

case "0":

Hex = "0001";

break;

case "1":

Hex = "0002";

break;

case "2":

Hex = "0004"; 
break;

case "3":

Hex = "0008";

break;

case "4":

Hex = "0010";

break;

case "5":

Hex = "0020";

break;

case "6":

Hex = "0040";

break;

case "7":

Hex = "0080";

break;

case "8":

Hex = "0100";

break;

case "9":

Hex = "0200";

break;

case "10":

Hex = "0400";

break;

case "11":

Hex = "0800";

break; 
case "12":

Hex = "1000";

break;

case "13":

Hex = "2000";

break;

case "14":

Hex = "4000";

break;

case "15":

Hex = "8000";

break;

\}

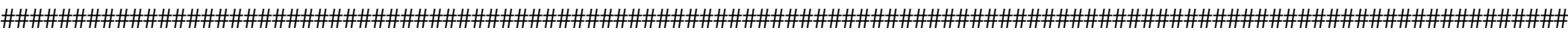

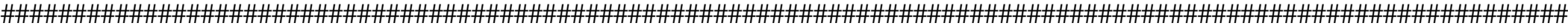
\# This funciton converts a 4 bit binary word into a hex value

\# Used in checking the event status word as more than one bit could be set $(0,1,2,3)$

\# Ex: bits $0,2,3=1101$ (binary) = D (hex)

static function Bit4_to_Hex1 (Bit, out Hex)

\{

auto Dec;

auto bit[];

auto i;

for $(\mathrm{i}=0 ; \mathrm{i}<$ length(Bit); $\mathrm{i}++)$

\{

bit $[\mathrm{i}]=\operatorname{substr}($ Bit, length(Bit)-i, 1);

$\operatorname{Dec}+=\operatorname{bit}[\mathrm{i}] * 2^{\wedge} \mathrm{i}$;

\}

Hex = sprintf("\%.2X", Dec);

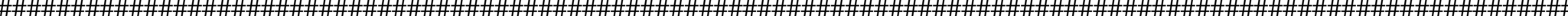

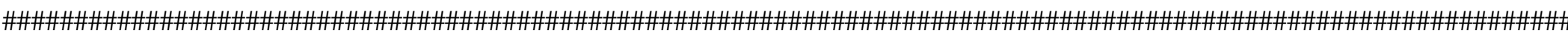


\#\#\#\#!!!!!HARDWARE CHECKOUT FUNCTIONS!!!!!\#\#\#\#

public function ATP_Hardware_Check ()

\{

auto i;

auto ODSC;

auto DDT_MODE_READ;

auto rc;

auto ODSC_Row;

auto value;

auto Output;

ddt_close_all_tables();

if (win_exists("Input Discretes - System2 - Virtual Panel", 1)==E_OK)

win_close("Input Discretes - System2 - Virtual Panel");

Startup_Virtual_Panels("Input Discretes - System2 - Virtual Panel");

\# Power Controls - Virtual Panel

set_window ("Power Controls - Virtual Panel", 2);

menu_select_item ("Panel;Load Configuration Ctrl+L");

\# Load a Configuration...

set_window ("Load a Configuration...", 1);

list_select_item ("ListBox", "Power Default"); \# Item Number 0;

button_press ("Load");

\# Analog Controls - Virtual Panel

set_window ("Analog Controls - Virtual Panel", 1);

menu_select_item ("Panel;Load Configuration Ctrl+L");

\# Load a Configuration...

set_window ("Load a Configuration...", 1);

list_select_item ("ListBox", "Analog Default"); \# Item Number 0;

button_press ("Load");

if (win_exists("Air_Data HW RX", 1)!=E_OK)

ATP_Startup_ACO_Air_Data();

if (win_exists("GPS_Navigation HW RX", 1)!=E_OK)

ATP_Startup_ACO_GPS_Navigation();

\# Input Discretes - System2 - Virtual Panel

set_window ("Input Discretes - System2 - Virtual Panel", 2); 
list_select_item ("Mode Command", "Nav"); \# Item Number 1

set_window ("Output Discretes - Virtual Panel", 2);

obj_wait_info("Align ODSC", "enabled", 1, 15);

set_window ("Input Discretes - System2 - Virtual Panel", 2);

list_select_item ("Mode Command", "Power Off");

\#Check initial state of Output Doscretes to be Lit

ODSC = "I\ATP output discretes.xls";

$\mathrm{rc}=\mathrm{ddt}$ open(ODSC, DDT_MODE_READ);

if $\left(\mathrm{rc} !=\mathrm{E} \_\mathrm{OK} \& \& \mathrm{rc} !=\mathrm{E} \_\right.$FILE_OPEN)

pause("Cannot open table.");

for(ODSC_Row $=1$; ODSC_Row $<=17$; ODSC_Row ++ )

\{

ddt_set_row(ODSC,ODSC_Row);

Output $=$ ddt_val(ODSC, "Output");

set_window("Output Discretes - Virtual Panel", 1);

obj_get_info(Output,"enabled",value);

if $($ value $==1)$

Lit.");

tl_step("Check initial state of " Output ".", PASS, Output " was Lit.", Output " should be Lit.", Output " was

else

tl_step("Check initial state of " Output ".", FAIL, Output " was NOT Lit.", Output " should be Lit.", Output " was NOT Lit.");

\}

ddt_close(ODSC);

report_msg("Finished with Hardware Check Initialization.");

\# Call Hardware Check Functions

set_window ("ACO - GPS_Navigation HW RX", 1);

menu_select_item ("Configuration;Update All Rx Labels");

set_window ("ACO - Air_Data HW RX", 1);

menu_select_item ("Configuration;Update All Rx Labels");

ATP_Check_Air_Data_Resolvers();

set_window ("ACO - GPS_Navigation HW RX", 1);

menu_select_item ("Configuration;Update All Rx Labels"); 
set_window ("ACO - Air_Data HW RX", 1);

menu_select_item ("Configuration;Update All Rx Labels");

ATP_Check_Receivers("GPS_Navigation");

ATP_Check_Receivers("Air_Data");

ATP_Check_Input("GPS_Navigation");

ATP_Check_Input("Air_Data");

\#ATP_Check_Output_Discretes();

\#ATP_Check_Sensor_Assembly();

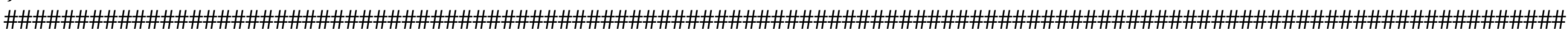

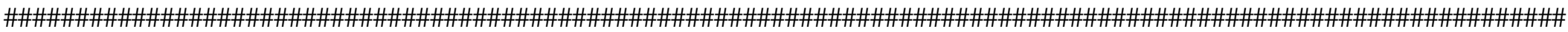
public function ATP_Startup_ACO_Air_Data()

auto value;

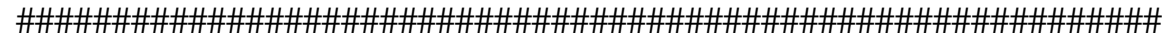

\#Check and start 8 channel transmitter

if(win_exists("ACO - Air_Data HW TX",1)==E_OK)

\{

set_window("ACO - Air_Data HW TX",1);

set_window ("Air_Data HW TX", 1);

button_set ("Start Transmitting", OFF);

\}

win_close("ACO - Air_Data HW TX");

invoke_application("I\ACO\\ACO.exe","",""',SW_SHOW);

\# Select an ARINC board

set_window ("Select an ARINC board", 4);

list_select_item ("SysListView32", "0"); \# Item Number 0;

button_press ("OK");

\# ACO 1

set_window ("ACO_1", 1);

button_press ("Yes");

\# Setup Page

set_window ("Setup Page", 2);

button_press ("Load"); 


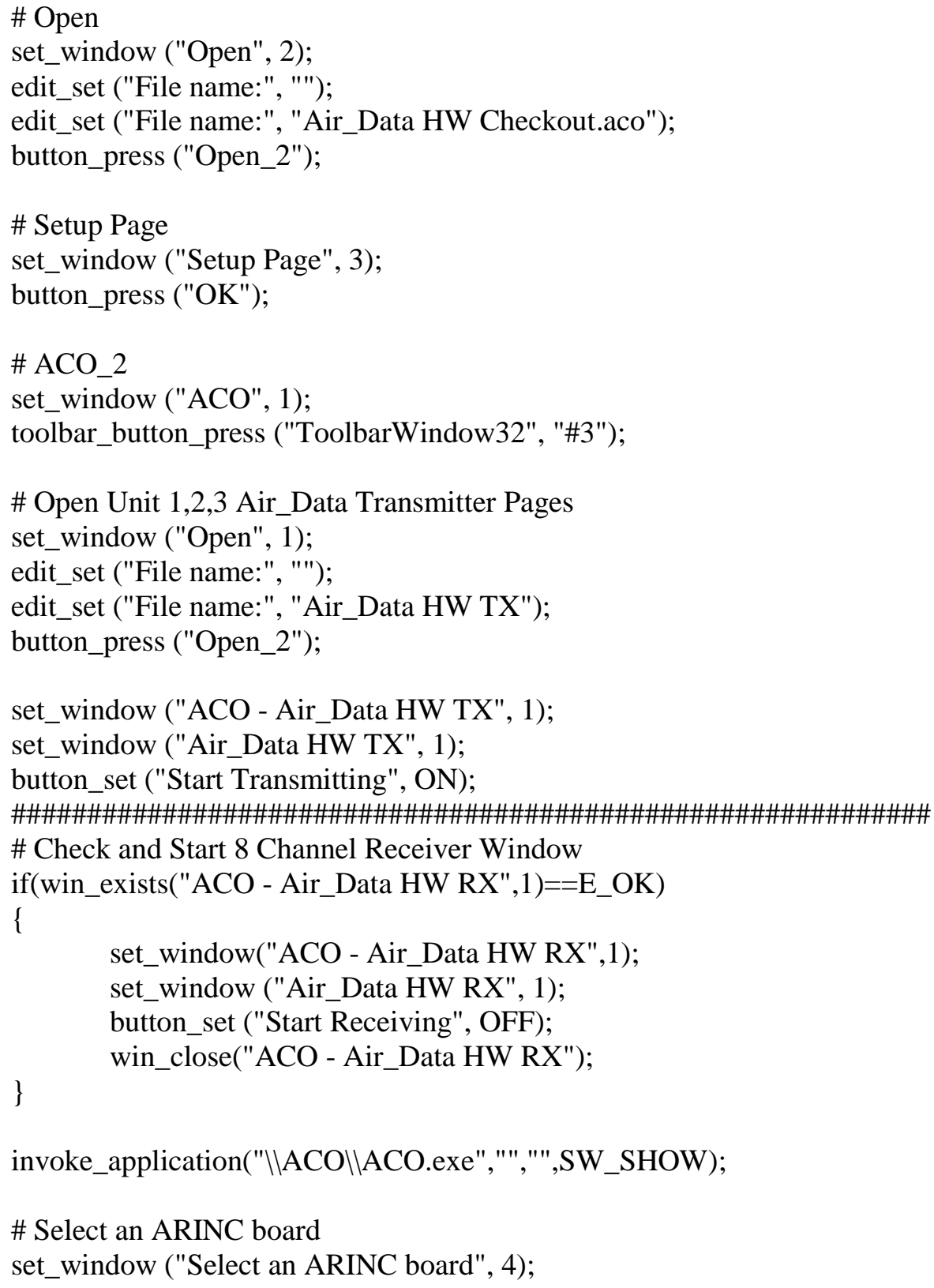


list_select_item ("SysListView32", "0"); \# Item Number 0; button_press ("OK");

\section{\# ACO_1}

set_window ("ACO_1", 1);

button_press ("Yes");

\# Setup Page

set_window ("Setup Page", 3);

button_press ("Cancel");

\# ACO

set_window ("ACO", 2);

toolbar_button_press ("ToolbarWindow32", "\#5");

\# Open

set_window ("Open", 3);

edit_set ("File name:", "");

edit_set ("File name:", "\Air_Data HW RX")

button_press ("Open_2");

\# Air_Data Receiver

set_window("ACO - Air_Data HW RX", 5);

set_window ("Air_Data HW RX", 4);

button_set ("Start Receiving", ON);

win_resize ("Air_Data HW RX", 861, 912);

win_move ("Air_Data HW RX", 0, 0);

set_window ("ACO - Air_Data HW RX", 1);

\}

menu_select_item ("Configuration;Update All Rx Labels");

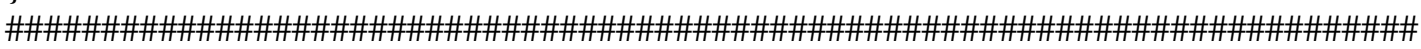

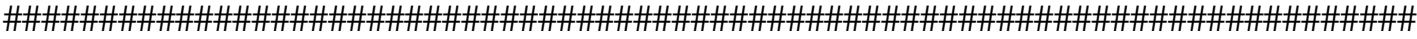
public function ATP_Startup_ACO_GPS_Navigation()

\{ auto value;

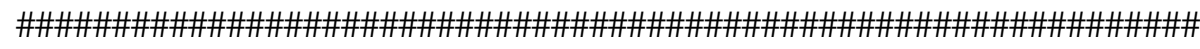

\#Check and start 16 channel transmitter

if(win_exists("ACO - GPS_Navigation HW TX",1)==E_OK)

\{

set_window("ACO - GPS_Navigation HW TX",1); 
set_window ("GPS_Navigation HW TX", 1);

button_set ("Start Transmitting", OFF);

win_close("ACO - GPS_Navigation HW TX");

invoke_application("\\ACOI\ACO.exe","'","',SW_SHOW);

\# Select an ARINC board

set_window ("Select an ARINC board", 4);

list_select_item ("SysListView32", "1"); \# Item Number 0;

button_press ("OK");

\# ACO_1

set_window ("ACO_1", 1);

button_press ("Yes");

\# Setup Page

set_window ("Setup Page", 2);

button_press ("Load");

\# Open

set_window ("Open", 2);

edit_set ("File name:", "");

edit_set ("File name:", "IGPS_Navigation HW Checkout.aco");

button_press ("Open_2");

\# Setup Page

set_window ("Setup Page", 1);

button_press ("OK");

\# ACO_2

set_window ("ACO", 1)

toolbar_button_press ("ToolbarWindow32", "\#3");

\# Open Navigation Transmitter Page

set_window ("Open", 1);

edit_set ("File name:", "");

edit_set ("File name:", "IGPS_Navigation HW TX");

button_press ("Open_2"); 
set_window ("ACO - GPS_Navigation HW TX", 1);

set_window ("GPS_Navigation HW TX", 1);

button_set ("Start Transmitting", ON);

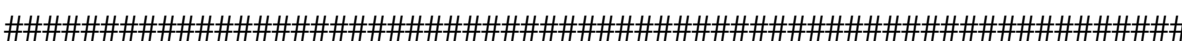

\# Check and Start 16 Channel Receiver Window

if(win_exists("ACO - GPS_Navigation HW RX",1)==E_OK)

\{

set_window("ACO - GPS_Navigation HW RX",1)

set_window ("GPS_Navigation HW RX", 1);

button_set ("Start Receiving", OFF);

win_close("ACO - GPS_Navigation HW RX");

invoke_application("\\ACO\\ACO.exe","","',SW_SHOW);

\# Select an ARINC board

set_window ("Select an ARINC board", 4);

list_select_item ("SysListView32", "1"); \# Item Number 0;

button_press ("OK");

\# ACO_1

set_window ("ACO_1", 1);

button_press ("Yes");

set_window ("Setup Page", 3);

button_press ("Cancel");

\# ACO

set_window ("ACO", 2);

toolbar_button_press ("ToolbarWindow32", "\#5");

\# Open

set_window ("Open", 3);

edit_set ("File name:", "");

edit_set ("File name:", "\GPS_Navigation HW RX");

button_press ("Open_2");

\# Navigation Receiver

set_window("ACO - GPS Navigation HW RX", 1);

set_window ("GPS_Navigation HW RX", 4); 
button_set ("Start Receiving", ON);

win_resize ("GPS_Navigation HW RX", 861, 911);

win_move ("GPS_Navigation HW RX", 0, 0);

set_window ("ACO - GPS_Navigation HW RX", 1);

\}

menu_select_item ("Configuration;Update All Rx Labels");

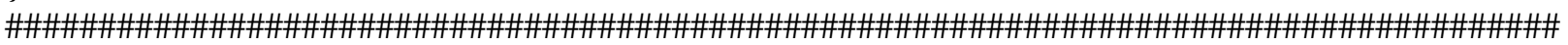

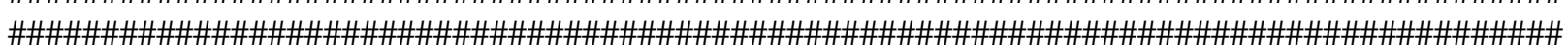
public function ATP_Check_Receivers(Function)

\{

auto i;

auto Port;

auto Label;

auto RealValue;

auto ExpectedValue;

auto rate;

auto rc;

auto DDT_MODE_READ;

auto Transmitters;

auto Transmitters_Row;

auto Receiver;

auto Transmitters_RowCount;

if (Function == "GPS_Navigation")

\{

Transmitters_RowCount $=34$;

$$
\}
$$

Transmitters = "ATP GPS_Navigation transmitter data.xls";

Transmitters_RowCount $=32$;

Transmitters = "ATP Air_Data transmitter data.xls";

\}

rc $=$ ddt_open(Transmitters, DDT_MODE_READ);

if (rc!= E_OK \& \& rc != E_FILE_OPEN)

pause("Cannot open table."); 
for(Transmitters_Row $=1$; Transmitters_Row $<=$ Transmitters_RowCount; Transmitters_Row ++$)$ \{

ddt_set_row(Transmitters,Transmitters_Row);

Receiver $=$ ddt_val(Transmitters, Function "_Receiver");

Port $=$ ddt_val(Transmitters, Function "_Port");

Label $=$ ddt_val(Transmitters, "Label");

ExpectedValue $=$ ddt_val(Transmitters, "Expected_Value");

Check_Label_Value_With_Mask("Check " Receiver, Function " ATP", Port, Label, ExpectedValue,"111111111111111111111111");

\}

report_msg("End of " Function " Transmitter/Receiver Test Function"); ddt_close(Transmitters);

\}

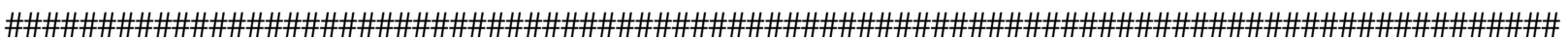

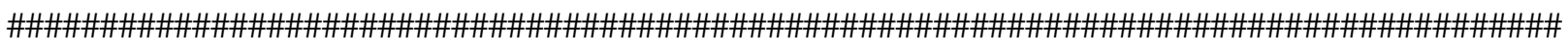
public function ATP_Check_Input(Function)

\{

auto IDSC;

auto rc;

auto DDT_MODE_READ;

auto IDSC_Row;

auto Label;

auto Discrete;

auto ExpectedValue;

auto Mask;

auto Ground;

auto Open;

auto Bit;

auto ActualBits;

auto RealValue;

auto i;

auto Port;

auto array[]; 
auto count[];

auto ReturnValue;

auto bits[];

auto Value;

auto Designation;

auto IDSC_RowCount;

auto tm1;

auto rate;

auto tm2;

auto TimeMark1;

auto TimeMark2;

auto TimeCount 1

auto TimeCount2;

auto Time1;

auto Time2;

auto bit[];

auto ATP_window;

if (Function == "GPS_Navigation")

\{

IDSC = "ATP GPS_Navigation input discretes.xls";

IDSC_RowCount $=36$;

\}

ATP_window = "GPS_Navigation HW RX";

else

\{

IDSC_RowCount $=33$;

IDSC = "ATP Air_Data input discretes.xls";

\}

ATP_window = "Air_Data HW RX";

$\mathrm{rc}=\mathrm{ddt} \_$open(IDSC,DDT_MODE_READ);

if (rc!= E_OK \&\& rc != E_FILE_OPEN)

pause("Cannot open table.");

\# Set Custom Values for Input Discretes

for(IDSC_Row $=1$; IDSC_Row <= IDSC_RowCount; IDSC_Row ++)

ddt_set_row(IDSC,IDSC_Row); 
Label = ddt_val(IDSC, "Label");

Discrete $=$ ddt_val(IDSC, "Signal_Reference");

ExpectedValue = ddt_val(IDSC, "Expected_with_Ground");

Designation $=$ ddt_val(IDSC, "Designation");

Port = ddt_val(IDSC, "Port");

Mask $=$ ddt_val(IDSC, "Mask");

Ground = ddt_val(IDSC, "Button_Ground");

Open = ddt_val(IDSC, "Button_Open");

Bit = ddt_val(IDSC, "Bit");

if (Discrete $==$ "Aircraft Parity")

wait(2);

split(Bit, count, ",");

\# Input Discretes - Virtual Panel

set_window ("Input Discretes - System2 - Virtual Panel", 3);

list_select_item (Discrete, "*Custom");

\# Program Write Enable : Custom Pin Setting

set_window ( Discrete " : Custom Pin Setting", 1);

button_set (Ground, ON);

button_press ("OK");

if ( count[2])

\{

wait(2);

set_window (ATP_window, 5);

obj_wait_info("SysListView32","displayed",1,10);

list_activate_item ("SysListView32", Port ";" Label);

if(win_exists("Label Data...", 1)!= E_OK)

list_activate_item ("SysListView32", Port ";" Label);

set_window("Label Data...", 1);

obj_get_text("Rate", rate); 
obj_get_text("Value", RealValue);

win_close ("Label Data...");

if (rate $==0$ ) \#Label is not transmitted

\{

tl_step("Check " Function " Label " Label, FAIL, Function " Label " Label " Could not be checked, it was not transmitted.", Function " Label " Label " should be transmitted" , Function " Label " Label " Was not transmitted");

\} continue;

if (rate >=1) \#Check Label Value to expected

\{

RealValue = repl_str(RealValue," ","'");

\# Convert ACO Bits String to Actual Bits set

for (i=0; i < length(RealValue); i++)

\{

$\operatorname{array}[i+1]=\operatorname{substr}($ RealValue, $i+1,1)$;

if $(\operatorname{array}[i+1]==0)$

$\operatorname{array}[\mathrm{i}+1]=32-\mathrm{i}$ ", ";

else

$\operatorname{array}[i+1]=" " ;$

\}

\# Labels 100-103 are GPS_Navigation, Labels 110-112 are Air_Data

if $($ Label $==100)$

ActualBits = "Bit(s) " array[21] array[20] array[19] array[18] array[14] array[13] array[12] array[11] array [10] array[9];

if $($ Label $==101)$

ActualBits = "Bit(s) " array[22] array[21] array[19] array[13] array[12] array[11] array[10] array[9] array[8];

if (Label $==102$ )

ActualBits = "Bit(s) " array[22] array[21] array[20] array[19] array[13] array[12] array[11] array[10] array[9];

if $($ Label $==103)$

ActualBits = "Bit(s) " array[22] array[21] array[20] array[14] array[13] array[12] array[9];

if $($ Label $==110)$ 
array[7];

ActualBits = "Bit(s) " array[22] array[21] array[20] array[19] array[18] array[17] array[16] array[15] array[14] array[13] array[12] array[11] array[10] array[9] array[8] array[7];

if $($ Label $==111)$

ActualBits = "Bit(s) " array[22] array[13] array[12] array[11] array[10] array[9] array[8]

if $($ Label $==112)$

ActualBits = "Bit(s) " array[19] array[14] array[13] array[12] array[11] array[10] $\operatorname{array[9]}$ array[8] array[7];

WRU_MaskAndCompareBinString(ExpectedValue, RealValue, Mask, ReturnValue);

if $($ ReturnValue $==1)$

tl_step("Check " Function " Label " Label, PASS, "All Input Discretes on " Function " Label " Label " were grounded correctly. Expected: Bit(s) " Bit " set on " Function " Label " Label ". Actual: " ActualBits, "Expected: Bit(s) " Bit " set on " Function " Label " Label, "Actual: " ActualBits);

else

tl_step("Check " Function " Label " Label, FAIL, "All Input Discretes on " Function " Label " Label " were NOT grounded correctly. Expected: Bit(s) " Bit " set on " Function " Label " Label ". Actual: " ActualBits, "Expected: Bit(s) " Bit " set on " Function " Label " Label, "Actual: " ActualBits);

\}

\}

\# END GPS_Navigation DISCRETE CHECK LOOP

\# Check GPS Time Mark Count

if (Function == "GPS_Navigation")

\{

for $(\mathrm{i}=1 ; \mathrm{i}<=100 ; \mathrm{i}++)$

\{

set_window ("GPS_Navigation HW RX", 5);

list_activate_item ("SysListView32", "6;106");

if (win_exists("Label Data...", 1)!=E_OK)

list_activate_item ("SysListView32", "6;106");

set_window("Label Data...", 1); 


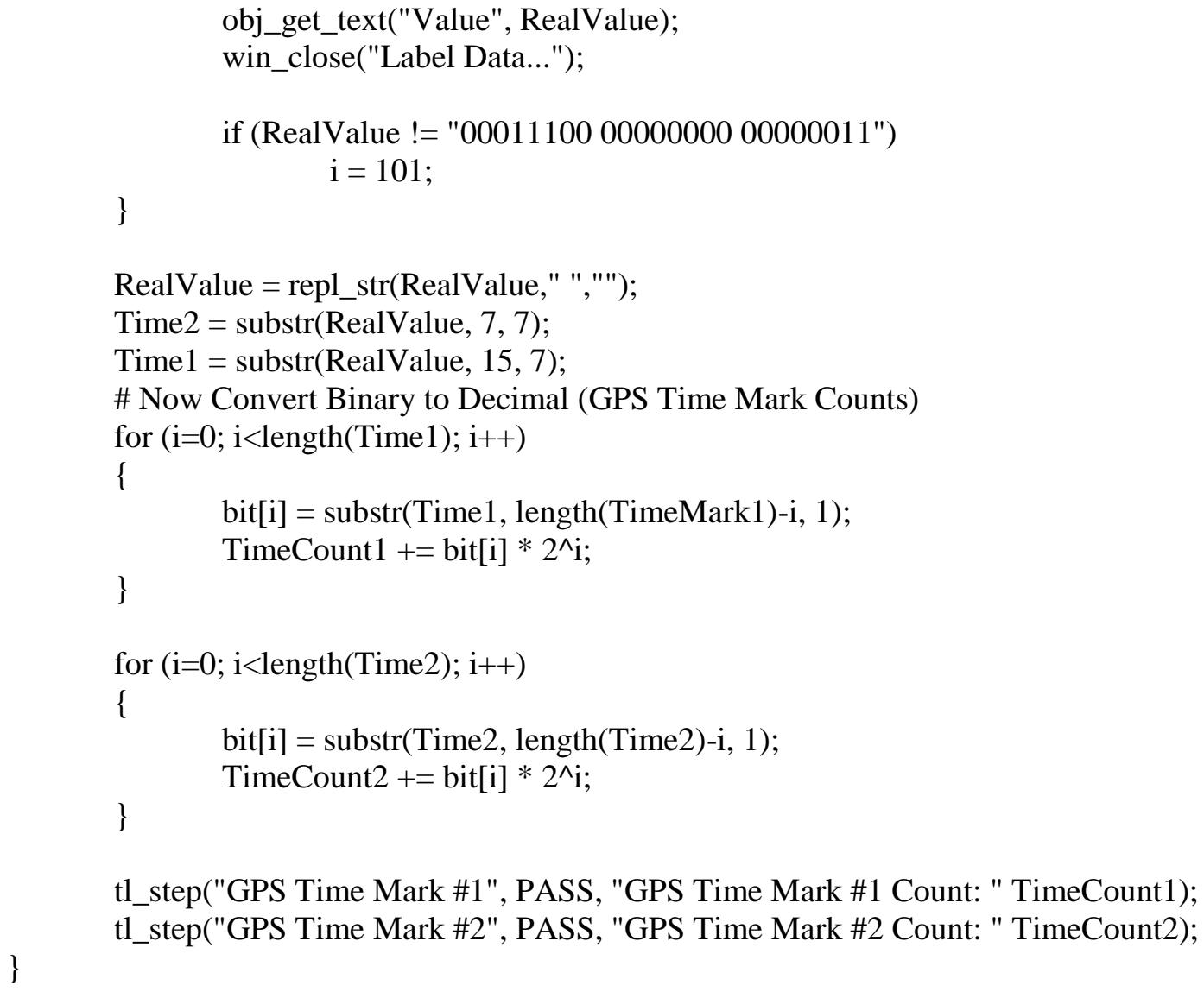

report_msg("End of GPS_Navigation Input Discrete Test Function"); ddt_close(IDSC); 
auto Value;

auto Label;

auto ExpectedValue;

auto Designation;

auto ResolverValue;

auto RealValue;

auto ResolverValueDec;

auto TwosComp;

auto RealVoltage;

auto SetValue;

auto rc;

auto i;

auto Resolvers_Row;

auto rate;

auto Angle;

auto bits[];

auto bit[];

auto twos[];

auto Units;

auto Port;

auto MaxVoltage = 10;

auto Tolerance;

auto ResRef;

auto Function;

\# Input Discretes - System2 - Virtual Panel

set_window ("Input Discretes - System2 - Virtual Panel", 2);

list_select_item ("26 VAC Ref 1", "Reference On"); \# Item Number 1;

list_select_item ("26 VAC Ref 2", "Reference On"); \# Item Number 1;

\# Analog Controls - Virtual Panel

set_window ("Analog Controls - Virtual Panel", 4);

menu_select_item ("Panel;Load Configuration Ctrl+L");

\# Load a Configuration...

set_window ("Load a Configuration...", 0);

list_select_item ("ListBox", "ATP"); \# Item Number 1;

button_press ("Load"); 
\# Get Reference Voltage Reading

win_move ("Power Controls - Virtual Panel", 127, 33);

set_window ("Power Controls - Virtual Panel", 2);

list_select_item ("Volt Reader", "Resolver Ref"); \# Item Number 7;

wait(8);

obj_get_text("Value:", ResRef);

win_move ("Power Controls - Virtual Panel", 1482, 617);

if $($ ResRef $>23 \& \&$ ResRef $<28)$

tl_step("Check Resolver Reference.", PASS, "Resolver Reference is within operational range. Expected Range: 23-28 vac RMS. Actual Voltage: " ResRef " vac RMS.", "Expected Range: 23-28 vac RMS.", "Actual Voltage: " ResRef " vac

RMS.");

else

tl_step("Check Resolver Reference.", FAIL, "Resolver Reference is NOT within operational range. Expected Range: 2328 vac RMS. Actual Voltage: " ResRef " vac RMS.", "Expected Range: 23-28 vac RMS.", "Actual Voltage: " ResRef " vac RMS.");

\# Analog Controls - Virtual Panel

win_move ("Analog Controls - Virtual Panel", 1305, 819);

Resolvers = "ATP Air_Data resolvers.xls";

$\mathrm{rc}=$ ddt_open(Resolvers, DDT_MODE_READ);

if $\left(\mathrm{rc} !=\mathrm{E} \_\mathrm{OK} \& \& \mathrm{rc} !=\right.$ E_FILE_OPEN)

pause("Cannot open table.");

for $($ Resolvers_Row $=1$; Resolvers_Row $<=20$; Resolvers_Row ++ )

\{

ddt_set_row(Resolvers,Resolvers_Row);

Label = ddt_val(Resolvers, "Label");

Designation = ddt_val(Resolvers, "Designation");

ExpectedValue = ddt_val(Resolvers, "Expected_Value_Dec");

Units = ddt_val(Resolvers, "Units");

Angle $=$ ddt_val(Resolvers, "Angle");

Port $=$ ddt_val(Resolvers, "Port");

ResolverValue = "";

TwosComp = "";

Function = "Air_Data";

for $(\mathrm{i}=1 ; \mathrm{i}<=10 ; \mathrm{i}++)$ 
set_window ("Air_Data HW RX", 5);

obj_wait_info("SysListView32","displayed",1,10);

list_activate_item ("SysListView32", Port ";" Label);

if(win_exists("Label Data...", 1)!=E_OK)

list_activate_item ("SysListView32", Port ";" Label);

set_window("Label Data...", 1);

obj_get_text("Rate", rate);

obj_get_text("Value", RealValue);

win_close ("Label Data...");

if (rate $<1 \& \& \mathrm{i}<10$ ) \#Try again due to Update Delay in ACO

continue;

if (rate $<1 \& \& \mathrm{i}==10$ ) \#Label is not transmitted

tl_step("Check " Function " Label " Label, FAIL, Function " Label " Label " Could not be checked, it was not transmitted.", Function " Label " Label " should be transmitted" , Function " Label " Label " Was not transmitted");

\# Check Resolver Values

if $($ rate $>=1)$

\{

RealValue = repl_str(RealValue," ","');

ResolverValue = substr(RealValue, 7, 16);

\# Need Twos Compliment if MSB is 1

if $(\operatorname{substr}($ RealValue, 7,1$)==1)$

for $(\mathrm{i}=2 ; \mathrm{i}<=16 ; \mathrm{i}++)$

\{

twos[i] = substr(ResolverValue, i, 1);

if $(\operatorname{twos}[\mathrm{i}]==1)$

$\operatorname{twos}[\mathrm{i}]=0$;

$\operatorname{twos}[\mathrm{i}]=1$; 
\}

ResolverValue $=$ TwosComp;

\# Now Convert Binary to Decimal and Multiply by $305 \mathrm{uV}$

ResolverValueDec $=0$;

for ( $\mathrm{i}=0$; $\mathrm{i}<$ length(ResolverValue); $\mathrm{i}++$ )

\{

bit[i] = substr(ResolverValue, length(ResolverValue $)$-i, 1);

ResolverValueDec $+=$ bit $[\mathrm{i}] * 2^{\wedge} \mathrm{i}$

RealVoltage $=$ ResolverValueDec $* .000305 ;$

if $(\operatorname{substr}($ RealValue, 7,1$)==1)$

RealVoltage $=($ ResolverValueDec $*-0.000305)-.000305$;

if $($ Resolvers_Row $<=8$ )

if (ExpectedValue-MaxVoltage*. $1<$ RealVoltage \&\& RealVoltage <

ExpectedValue+MaxVoltage*.1)

tl_step("Check " Designation, PASS, Designation " was set to within $10 \%$ Tolerance at Angle " Angle "on Air_Data ODB \#" Port ". Actual voltage: " RealVoltage " " Units ". Expected Voltage: "ExpectedValue " " Units, "Expected Voltage: " ExpectedValue " " Units, "Actual Voltage: " RealVoltage " " Units);

else

tl_step("Check " Designation, FAIL, Designation " was NOT within 10\% Tolerance at Angle " Angle "० on Air_Data ODB \#" Port ". Actual voltage: " RealVoltage " " Units ". Expected Voltage: "ExpectedValue " " Units, "Expected Voltage: " ExpectedValue " " Units, "Actual Voltage: " RealVoltage " " Units);

if (Resolvers_Row $>8$ \&\& Resolvers_Row $<=18$ )

\{

if (ExpectedValue-MaxVoltage*.1 < RealVoltage \&\& RealVoltage <

ExpectedValue+MaxVoltage*.1) 


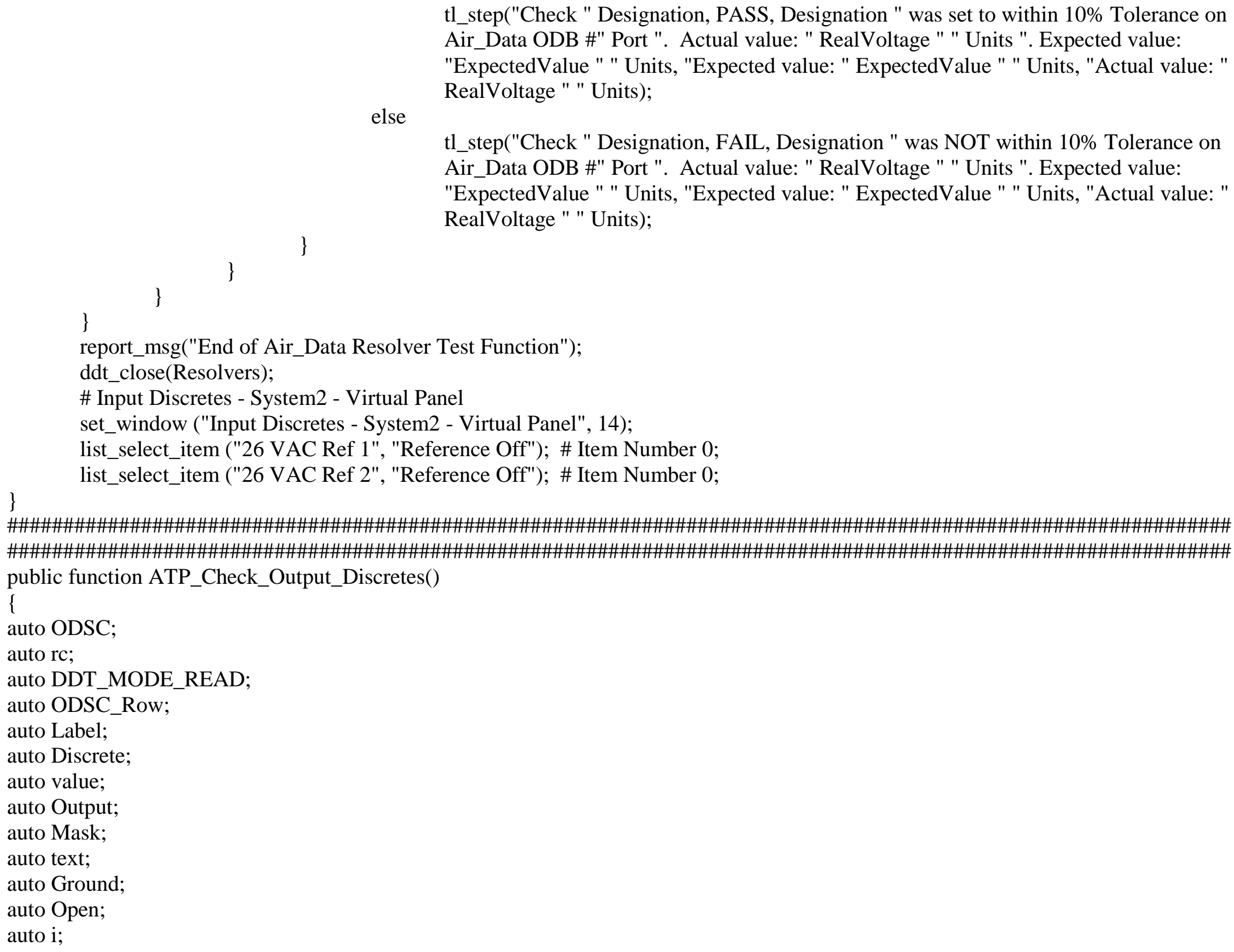


auto Designation;

auto ODSC_RowCount $=12$;

auto Batt;

auto DC;

auto Navigation;

auto Align;

auto Function;

auto Expected;

auto Port;

auto RealValue;

auto array[];

auto Bit[];

auto Setting;

auto rate;

ODSC = "ATP output discretes.xls";

rc $=$ ddt_open(ODSC, DDT_MODE_READ);

if ( $\mathrm{rc} !=\mathrm{E} \_\mathrm{OK} \& \& \mathrm{rc} !=$ E_FILE_OPEN)

pause("Cannot open table.");

\# Need to Ground A/C Discrete 1 to Test ODSC's affected by ANN Test Discrete set_window ("Input Discretes - System2 - Virtual Panel", 2);

list_select_item ("Aircraft Identification", "*Custom"); \# Item Number 20;

set_window ("Aircraft Identification : Custom Pin Setting", 0);

button_set ("Ground_6", ON);

button_press ("OK");

\#Check Output Discretes with Input Enabled

for(ODSC_Row $=1$; ODSC_Row < = ODSC_RowCount; ODSC_Row ++)

\{

ddt_set_row(ODSC,ODSC_Row);

Discrete $=$ ddt_val(ODSC, "Input");

Output = ddt_val(ODSC, "Output");

Ground = ddt_val(ODSC, "Button_Ground");

Open = ddt_val(ODSC, "Button_Open");

Designation $=$ ddt_val(ODSC, "Designation");

Function = ddt_val(ODSC, "Function");

Label = ddt_val(ODSC, "Label");

Expected = ddt_val(ODSC, "Expected_Bit"); 
Port $=$ ddt_val(ODSC, "Port");

Setting = ddt_val(ODSC, "Setting");

set_window("Output Discretes - Virtual Panel", 1);

obj_get_info(Output,"enabled",value);

if $($ value $==0)$

tl_step("Check " Discrete " " Designation " sets " Output, PASS, Output " was set correctly by Input Discrete "

Discrete " " Designation, "Expected: " Output " set.", "Actual: " Output " set.");

else

tl_step("Check " Discrete " " Designation " sets " Output, FAIL, Output " was NOT set correctly by Input

Discrete " Discrete " " Designation, "Expected: " Output " set.", "Actual: " Output " NOT set.");

if (length(Function) $>1$ )

set_window (Function " HW RX", 5);

obj_wait_info("SysListView32","displayed",1,10);

list_activate_item ("SysListView32", Port ";" Label);

if(win_exists("Label Data...", 1)!= E_OK)

list_activate_item ("SysListView32", Port ";" Label);

set_window("Label Data...", 1);

obj_get_text("Rate", rate);

obj_get_text("Value", RealValue);

win_close ("Label Data...");

if (rate $==0)$ \#Label is not transmitted

\{

tl_step("Check " Function " Label " Label, FAIL, Function " Label " Label " Could not be checked, it was not transmitted.", Function " Label " Label " should be transmitted" , Function " Label " Label " Was not transmitted"); continue;

if (rate $>0)$

\{

RealValue = repl_str(RealValue," ","'); 
\# Convert ACO Bits String to Actual Bits set

for (i=length(RealValue); $>$ > ; - -)

\{

\}

$\operatorname{array}[33-\mathrm{i}]=\operatorname{substr}($ RealValue, $\mathrm{i}, 1)$;

if $($ array[Expected $]==$ Setting $)$

tl_step("Check " Discrete " " Designation " sets " Output " W/A", PASS, Output " W/A was set on " Function " Label " Label " as Bit " Expected ".");

else

tl_step("Check " Discrete " " Designation " sets " Output " W/A", FAIL, Output " W/A was NOT set on " Function " Label " Label " as Bit " Expected ".");

\}

\# Check ANN Test Discrete by Setting A/C ID 1 to Ground

\# On Batt, Low DC, Navigation Warn, Align should be enabled with A/C ID 8, GPS Priority Select

\# Input Discretes - System2 - Virtual Panel

set_window ("Input Discretes - System2 - Virtual Panel", 4);

list_select_item ("Aircraft Identification", "*Custom");

set_window ("Aircraft Identification : Custom Pin Setting", 0);

button_set ("Ground_3", ON);

button_press ("OK");

\# Input Discretes - System2 - Virtual Panel

set_window ("Input Discretes - System2 - Virtual Panel", 8);

list_select_item ("GPS Priority Select", "*Custom");

set_window ("GPS Priority Select : Custom Pin Setting", 0);

button_set ("Ground", ON);

button_press ("OK");

wait(2);

set_window("Output Discretes - Virtual Panel", 1);

obj_get_info("On Batt ODSC","enabled",Batt);

obj_get_info("Low DC ODSC","enabled",DC);

obj_get_info("Navigation Warn ODSC","enabled",Navigation);

obj_get_info("Align ODSC","enabled",Align); 
if $($ Batt $==0 \& \&$ DC $==0 \& \&$ Navigation $==0 \& \&$ Align $==0)$

tl_step("Check A/C ID \#1 sets ANN Test", PASS, "ANN Test was set correctly by A/C ID \#1.");

else

tl_step("Check A/C ID \#1 sets ANN Test", FAIL, "ANN Test was NOT set correctly by A/C ID \#1.");

\# Power Controls - Virtual Panel

\# Check Air_Data Warn with DC Present BR

set_window ("Power Controls - Virtual Panel", 5);

scroll_drag_from_min ("0.00_0", HSCROLL, 0);

wait(3);

set_window("Output Discretes - Virtual Panel", 1);

obj_get_info("Air_Data Warn ODSC","enabled",value);

if (value $==0)$

tl_step("Check DC Present BR sets Air_Data Warn", PASS, "Air_Data Warn was set correctly by DC Present BR.");

else

tl_step("Check DC Present BR sets Air_Data Warn", FAIL, "Air_Data Warn was NOT set correctly by DC Present BR."); set_window ("Air_Data HW RX", 5);

obj_wait_info("SysListView32","displayed",1,10);

list_activate_item ("SysListView32", "7;112" );

if(win_exists("Label Data...", 1)!= E_OK)

list_activate_item ("SysListView32", "7;112");

set_window("Label Data...", 1);

obj_get_text("Rate", rate);

obj_get_text("Value", RealValue);

win_close ("Label Data...");

if (rate $==0$ ) \#Label is not transmitted

tl_step("Check Air_Data Warn W/A on Air_Data Label", FAIL, "Air_Data Warn W/A Could not be checked, Air_Data

Label was not transmitted.");

if $($ rate $>=1)$

RealValue = repl_str(RealValue," ","');

\# Convert ACO Bits String to Actual Bits set

for (i=length(RealValue); $>$ > ; i--) 


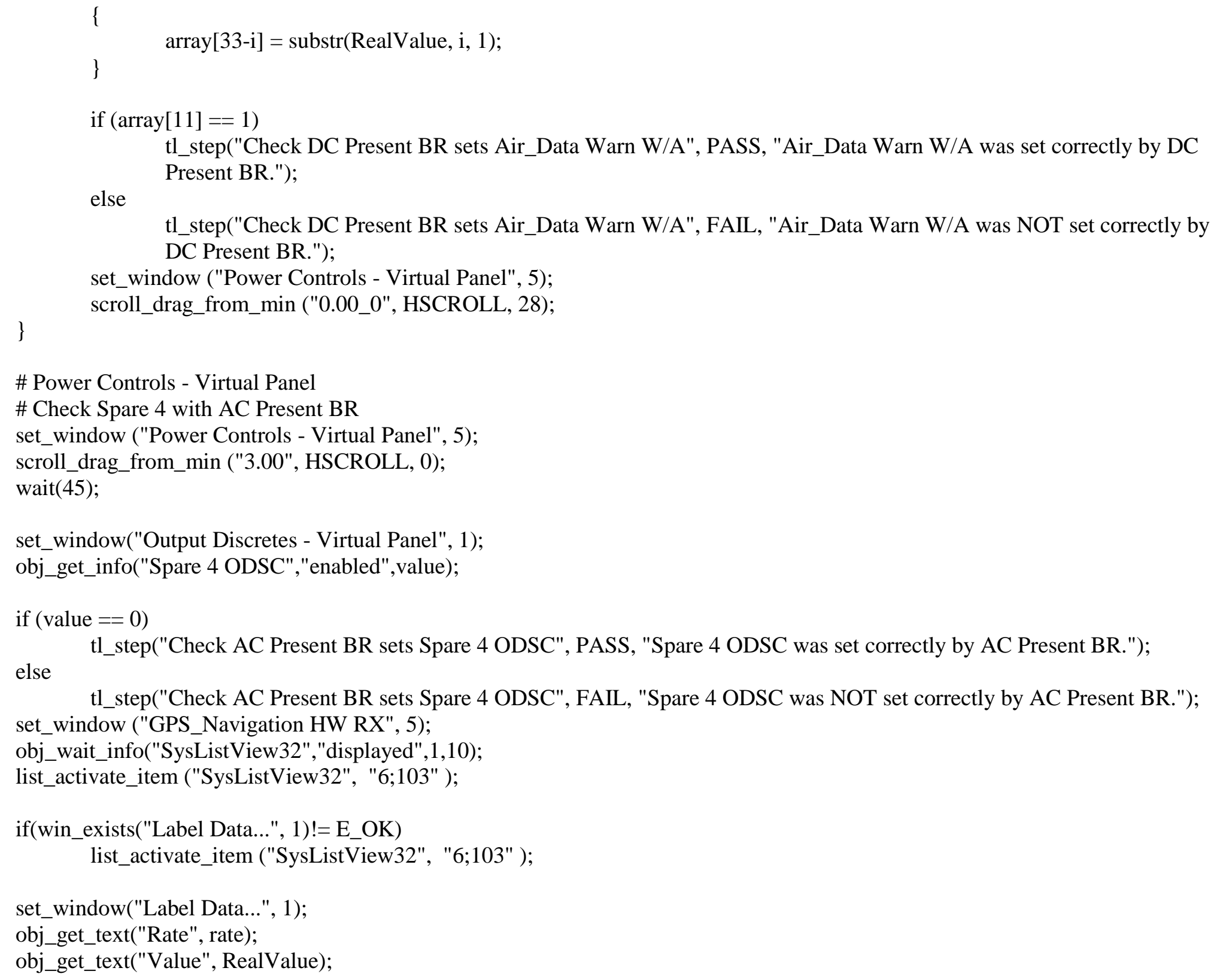




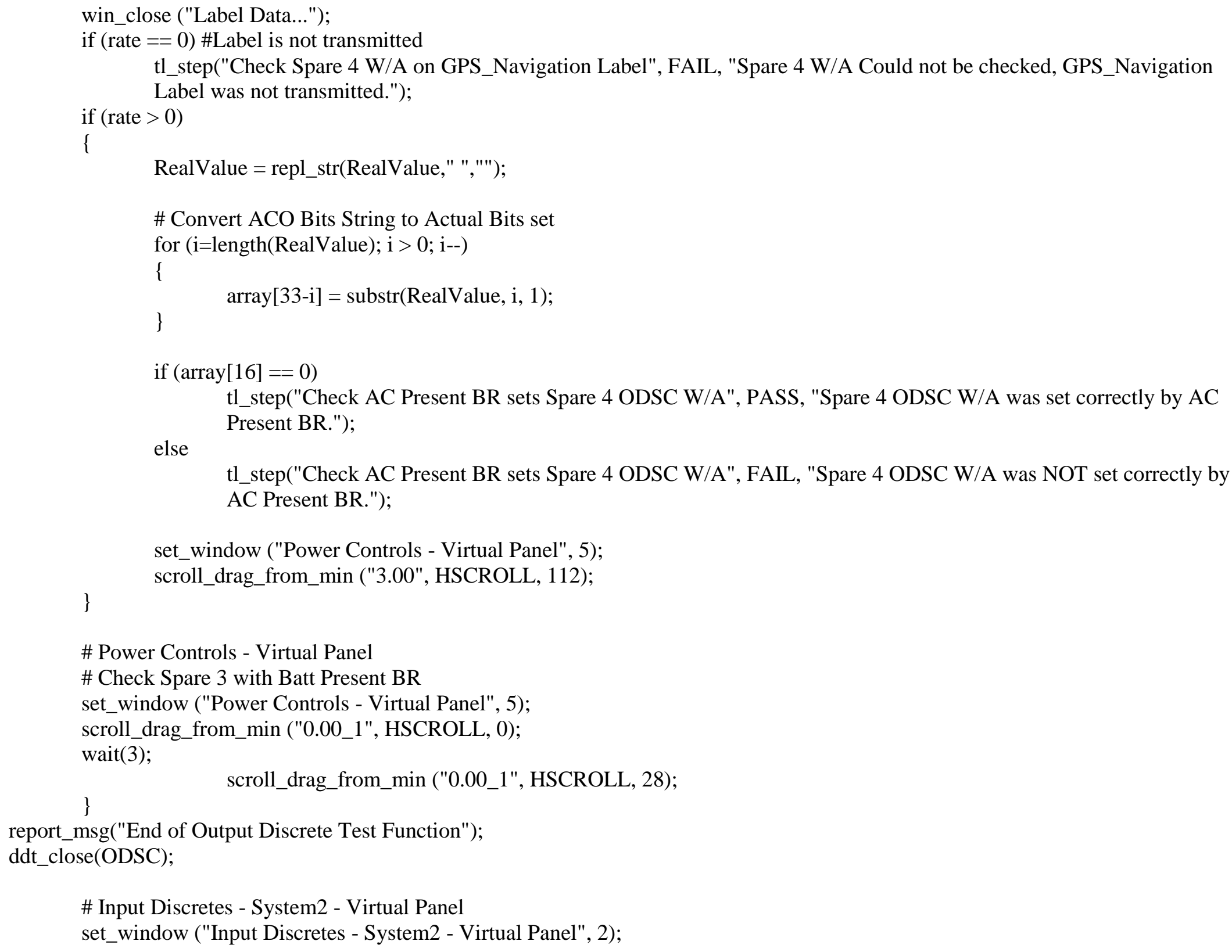

\# Input Discretes - System2 - Virtual Panel

set_window ("Input Discretes - System2 - Virtual Panel", 2); 
list_select_item ("Aircraft Identification", "*Custom"); \# Item Number 20;

\# Aircraft Identification : Custom Pin Setting

set_window ("Aircraft Identification : Custom Pin Setting", 0);

button_set ("Open_7", ON);

button_set ("Open_3", ON);

button_press ("OK");

\}

\#\#\#\#!!!!!END ATP HARDWARE CHECKOUT FUNCTIONS!!!!!\#\#\#\#

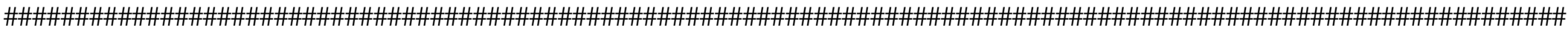

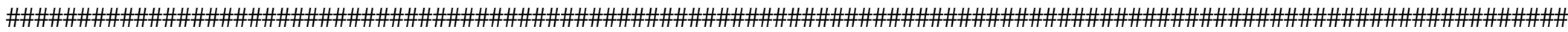
\#\#\#\#!!!!!EMI HARDWARE CHECKOUT FUNCTIONS!!!!!\#\#\#\#

\#\#\#\#\#\#\#\#\# EMI Hardware Checkout Startup\#\#\#\#\#\#\#\#

public function EMI_Hardware_Check (runtimes)

\{

auto i;

for $(\mathrm{i}=1 ; \mathrm{i}<=$ runtimes; $\mathrm{i}++)$

\{

ddt_close_all_tables();

if (win_exists("EMI Air_Data TX", 1)!=E_OK)

EMI_Startup_ACO_Air_Data();

if (win_exists("EMI GPS_Navigation TX", 1)!=E_OK)

EMI_Startup_ACO_GPS_Navigation();

EMI_Check_Receivers("GPS_Navigation");

EMI_Check_Input("GPS_Navigation");

EMI_Check_Receivers("Air_Data");

EMI_Check_Input("Air_Data");

EMI_Check_Air_Data_Resolvers();

EMI_Check_Output_Discretes();

EMI_Check_Sensor_Assembly();

\}

report_msg("Finished with Hardware Check (Run \#" runtimes ").");

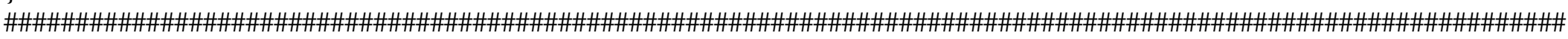

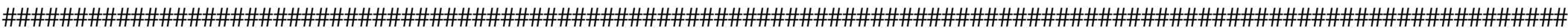
public function EMI_Startup_ACO_Air_Data() 
\{

auto value;

\#Check and start 8 channel Air_Data transmitter

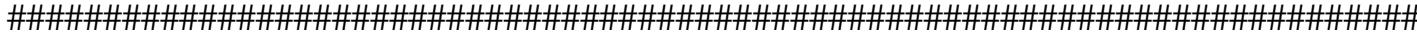

if(win_exists("ACO - EMI Air_Data TX",1)==E_OK)

\{

set_window ("Air_Data HW TX", 1);

button_set ("Start Transmitting", OFF);

\}

win_close("ACO - EMI Air_Data TX");

invoke_application("I\ACO\\ACO.exe","","'",SW_SHOW);

\# ACO_1

set_window ("ACO_1", 5);

button_press ("Yes")

\# Setup Page

set_window ("Setup Page", 2);

button_press ("Load");

\# Open

set_window ("Open", 2);

edit_set ("File name:", "");

edit_set ("File name:", "EMI ACO Setup.aco");

button_press ("Open_2");

\# Setup Page

set_window ("Setup Page", 3);

button_press ("OK");

\# ACO_2

set_window ("ACO", 1);

toolbar_button_press ("ToolbarWindow32", "\#3");

\# Open Air_Data Transmitter Page

set_window ("Open", 1);

edit_set ("File name:", "");

edit_set ("File name:", "EMI Air_Data TX"); 
button_press ("Open_2");

set_window ("EMI Air_Data TX", 1);

button_set ("Start Transmitting", ON);

\# Check and Start 8 Channel Air_Data Receiver Window

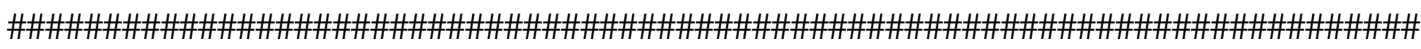
if(win_exists("ACO - EMI Air_Data RX",1)==E_OK)

\{

set_window ("Air_Data HW RX", 1);

button_set ("Start Receiving", OFF);

win_close("ACO - EMI Air_Data RX");

invoke_application("\\ACO\\ACO.exe","","",SW_SHOW);

\# ACO_1

set_window ("ACO_1", 0);

button_press ("Yes");

\# Setup Page

set_window ("Setup Page", 3);

button_press ("Cancel")

\# ACO

set_window ("ACO", 2);

toolbar_button_press ("ToolbarWindow32", "\#5");

\# Open

set_window ("Open", 3);

edit_set ("File name:", "");

edit_set ("File name:", "EMI Air_Data RX");

button_press ("Open_2");

set_window ("EMI Air_Data RX", 4);

button_set ("Start Receiving", ON);

win_resize ("EMI Air_Data RX", 924, 680);

win_move ("EMI Air_Data RX", 0, 0);

set_window ("ACO - EMI Air_Data RX", 2);

menu_select_item ("Configuration;Update All Rx Labels");

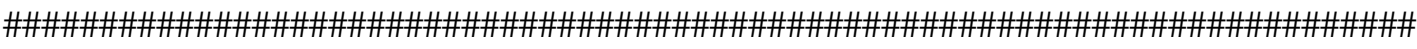

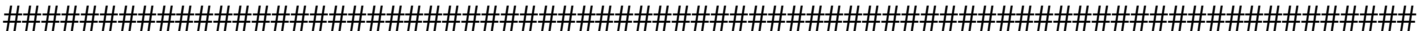
public function EMI_Startup_ACO_GPS_Navigation() 
auto value;

\#Check and start 8 channel GPS_Navigation transmitter

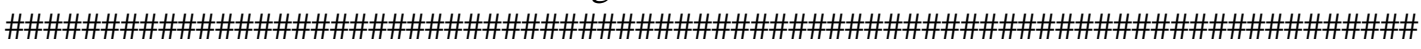
if(win_exists("ACO - EMI GPS_Navigation TX",1)==E_OK)

\{

set_window ("GPS_Navigation HW TX", 1);

button_set ("Start Transmitting", OFF);

\}

win_close("ACO - EMI GPS_Navigation TX");

invoke_application("I\ACO\\ACO.exe","","',SW_SHOW);

\section{\# ACO_1}

set_window ("ACO_1", 2);

button_press ("Yes");

\# Setup Page

set_window ("Setup Page", 2);

button_press ("Cancel");

\# ACO_2

set_window ("ACO", 1);

toolbar_button_press ("ToolbarWindow32", "\#3");

\# Open Unit 1,2,3 Air_Data Transmitter Pages

set_window ("Open", 1);

edit_set ("File name:", "");

edit_set ("File name:", "EMI GPS_Navigation TX");

button_press ("Open_2");

set_window ("EMI GPS_Navigation TX", 1);

button_set ("Start Transmitting", ON);

\# Check and Start 16 Channel Receiver Window

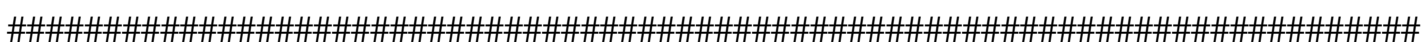

if(win_exists("ACO - EMI GPS_Navigation RX",1)==E_OK)

set_window ("GPS_Navigation HW RX", 1); 
button_set ("Start Receiving", OFF);

win_close("ACO - EMI GPS_Navigation RX");

invoke_application("\\ACO\\ACO.exe","'","',SW_SHOW);

\# ACO_1

set_window ("ACO_1", 0);

button_press ("Yes");

\# Setup Page

set_window ("Setup Page", 3);

button_press ("Cancel");

\# ACO

set_window ("ACO", 2);

toolbar_button_press ("ToolbarWindow32", "\#5");

\# Open

set_window ("Open", 3);

edit_set ("File name:", "");

edit_set ("File name:", "EMI GPS_Navigation RX");

button_press ("Open_2");

set_window ("EMI GPS_Navigation RX", 4);

button_set ("Start Receiving", ON);

win_resize ("EMI GPS_Navigation RX", 924, 680);

win_move ("EMI GPS_Navigation RX", 0, 0);

set_window ("ACO - EMI GPS_Navigation RX", 2);

\}

menu_select_item ("Configuration;Update All Rx Labels");

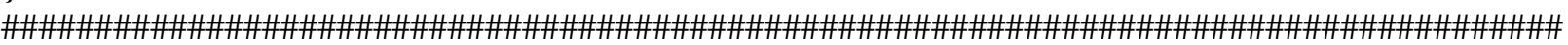

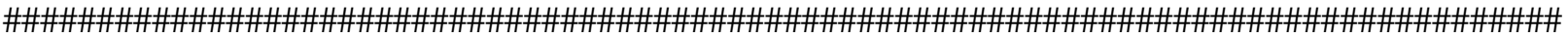
public function EMI_Check_Receivers(Function)

auto Port;

auto Label;

auto RealValue;

auto ExpectedValue;

auto rate;

auto rc; 
auto DDT_MODE_READ;

auto Transmitters;

auto Transmitters_Row;

auto Receiver;

auto Transmitters_RowCount $=20$;

if (Function == "GPS_Navigation")

\{

Transmitters_RowCount $=20$;

\}

Transmitters = "EMI GPS_Navigation transmitter data.xls";

else

\{

Transmitters_RowCount $=22$;

\}

Transmitters = "EMI Air_Data transmitter data.xls";

rc $=$ ddt_open(Transmitters, DDT_MODE_READ);

if $\left(\mathrm{rc} !=\mathrm{E} \_\mathrm{OK} \& \& \mathrm{rc} !=\mathrm{E} \_\right.$FILE_OPEN $)$

pause("Cannot open table.");

for(Transmitters_Row $=1$; Transmitters_Row $<=$ Transmitters_RowCount; Transmitters_Row ++$)$

\{

ddt_set_row(Transmitters,Transmitters_Row);

Receiver $=$ ddt_val(Transmitters, Function "_Receiver");

Port $=$ ddt_val(Transmitters, Function "_Port");

Label = ddt_val(Transmitters, "Label");

ExpectedValue $=$ ddt_val(Transmitters, "Expected_Value");

Check_Label_Value_With_Mask("Check " Function " Label " Label, Function " EMI", Port, Label, ExpectedValue,"111111111111111111111111");

\}

report_msg("End of GPS_Navigation Transmitter/Receiver Test Function");

ddt_close(Transmitters);

\}

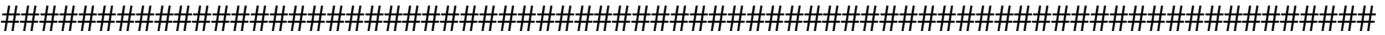

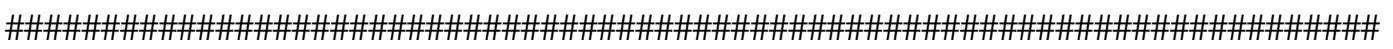


public function EMI_Check_Input(Function)

auto IDSC;

auto rc;

auto DDT_MODE_READ;

auto IDSC_Row;

auto Label;

auto Discrete;

auto ExpectedValue;

auto Mask;

auto Ground;

auto Open;

auto Bit;

auto ActualBits;

auto RealValue;

auto i;

auto array[];

auto ReturnValue;

auto bits[];

auto Value;

auto Designation;

auto IDSC_RowCount;

auto tm1;

auto tm2;

auto Port;

auto TimeMark1;

auto TimeMark2;

auto TimeCount1;

auto TimeCount2;

auto Time1;

auto Time2;

auto bit[];

auto EMI_window;

auto rate;

if (Function == "GPS_Navigation")

IDSC = "EMI GPS_Navigation input discretes.xls"; 
IDSC_RowCount $=4$;

EMI_window = "EMI GPS_Navigation RX";

\}

else

\{

IDSC_RowCount $=3$;

IDSC = "EMI Air_Data input discretes.xls";

EMI_window = "EMI Air_Data RX";

\}

IDSC = "EMI GPS_Navigation input discretes.xls";

rc = ddt_open(IDSC, DDT_MODE_READ);

if $\left(\mathrm{rc} !=\mathrm{E} \_\mathrm{OK} \& \& \mathrm{rc} !=\mathrm{E} \_\right.$FILE_OPEN)

pause("Cannot open table.");

for(IDSC_Row $=1$; IDSC_Row <= IDSC_RowCount; IDSC_Row ++)

\{

ddt_set_row(IDSC,IDSC_Row);

Label = ddt_val(IDSC, "Label");

ExpectedValue = ddt_val(IDSC, "Expected_with_Ground");

Mask $=$ ddt_val(IDSC, "Mask");

Bit $=$ ddt_val(IDSC, "Bit");

Port $=$ ddt_val(IDSC, "Port");

for $(\mathrm{i}=1 ; \mathrm{i}<=3 ; \mathrm{i}++)$

\{

set_window (EMI_window, 5);

obj_wait_info("SysListView32","displayed",1,10);

list_activate_item ("SysListView32", Port ";" Label);

if(win_exists("Label Data...", 1)!=E_OK)

list_activate_item ("SysListView32", Port ";" Label);

set window("Label Data...", 1);

obj_get_text("Rate", rate);

obj_get_text("Value", RealValue);

win_close ("Label Data..."); 
if (rate $<1 \& \& \mathrm{i}<3$ ) \#Try one more time due to Update Delay in ACO

continue;

if (rate $<1 \& \& \mathrm{i}==3$ ) \#Label is not transmitted

tl_step("Check " Function " Label " Label, FAIL, Function " Label " Label " Could not be checked, it was not transmitted.", Function " Label " Label " should be transmitted" , Function " Label " Label " Was not transmitted");

if (rate >=1) \#Check Label Value to expected

\{

RealValue = repl_str(RealValue," ","'");

\# Convert ACO Bits String to Actual Bits set

for $(\mathrm{i}=0 ; \mathrm{i}<$ length(RealValue); i++)

\{

$\operatorname{array}[i+1]=\operatorname{substr}($ RealValue, $i+1,1)$;

if $(\operatorname{array}[\mathrm{i}+1]==0)$

else

array $[i+1]=$ "bit " 32-i " ";

\}

$\operatorname{array}[\mathrm{i}+1]=$ "";

\#GPS_Navigation Labels are 100-103, Air_Data Labels are 110-112

if $($ Label $==100)$

ActualBits $=$ array[21] array[18] array[14] array[13] array[12] array[11] array[9];

if $($ Label $==101)$

ActualBits = array[21] array[14] array[13] array[12] array[11] array[10] array[9] array[8];

if (Label $==102)$

ActualBits = array[22] array[21] array[19] $\operatorname{array[13]~} \operatorname{array}[12]$ array[11] $\operatorname{array}[10]$ array[9];

if (Label $==103$ )

ActualBits = array[22] array[20] array[14] array[13] array[12] array[9];

if (Label $==110)$

ActualBits = array[22] array[21] array[20] array[19] array[18] array[16] array[15] array[14] array[13] array[12] array[11] array[10] array[9] array[8] array[7];

if $($ Label $==111)$

ActualBits = array[13] array[11] array[10] array[9] $\operatorname{array[8]~array[7];~}$

if $($ Label $==112)$ 
ActualBits = array[19] array[14] array[13] array[12] array[11] array[10] array[9] array[8] array[7];

WRU_MaskAndCompareBinString(ExpectedValue, RealValue, Mask, ReturnValue);

if $($ ReturnValue $==1)$

tl_step("Check " Function " Label " Label, PASS, "Discretes were set correctly on Label " Label " on " Function " ODB \#" Port ". Expected Ground: Bit " Bit " set on " Function " Label " Label ". Actual: " ActualBits, "Expected: Bit " Bit " set on " Function " Label " Label, "Actual: " ActualBits);

else

tl_step("Check " Function " Label " Label, FAIL, "Discretes were NOT set correctly on Label " Label " on " Function " ODB \#" Port ". Expected Ground: Bit " Bit " set on " Function " Label " Label ". Actual: " ActualBits, "Expected: Bit " Bit " set on " Function " Label " Label, "Actual: " ActualBits);

\}

break;

\# END GPS_Navigation DISCRETE CHECK LOOP

\# Check GPS Time Mark Count

if (Function == "GPS_Navigation")

\{

for $(\mathrm{i}=1 ; \mathrm{i}<=50 ; \mathrm{i}++)$

set_window ("GPS_Navigation HW RX", 5);

obj_wait_info("SysListView32","displayed",1,10);

list_activate_item ("SysListView32", "6;106");

if (win_exists("Label Data...", 1)==E_OK)

\{

\}

else

list_activate_item ("SysListView32", "6;106");

set_window("Label Data...", 1);

obj_get_text("Value", RealValue); 
win_close("Label Data...");

if (RealValue != "00011100 $0000000000000011 ")$

$$
\mathrm{i}=51 \text {; }
$$

\}

RealValue = repl_str(RealValue," ","');

Time2 = substr(RealValue, 7, 7);

Time1 = substr(RealValue, 15, 7);

\# Now Convert Binary to Decimal (GPS Time Mark Counts)

for $(\mathrm{i}=0 ; \mathrm{i}<$ length(Time1); $\mathrm{i}++)$

\{

$\operatorname{bit}[\mathrm{i}]=\operatorname{substr}($ Time1, length $($ TimeMark1 $)-\mathrm{i}, 1)$;

\}

TimeCount $1+=\operatorname{bit}[\mathrm{i}] * 2^{\wedge} \mathrm{i}$;

for $(\mathrm{i}=0 ; \mathrm{i}<$ length(Time2); i++)

\{

bit[i] = substr(Time2, length(Time2)-i, 1);

TimeCount $2+=\operatorname{bit}[\mathrm{i}] * 2^{\wedge} \mathrm{i}$;

\}

tl_step("GPS Time Mark \#1", PASS, "GPS Time Mark \#1 Count: " TimeCount1);

tl_step("GPS Time Mark \#2", PASS, "GPS Time Mark \#2 Count: " TimeCount2);

\}

report_msg("End of " Function " Input Discrete Test Function");

ddt_close(IDSC);

\}

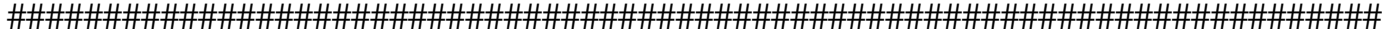

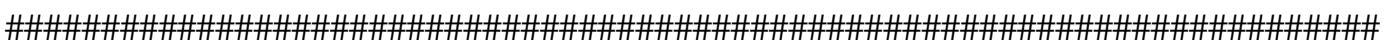

public function EMI_Check_Resolvers()

\{

auto Resolvers;

auto DDT_MODE_READ;

auto Control;

auto Value;

auto Label; 
auto ExpectedValue;

auto Designation;

auto ResolverValue;

auto RealValue;

auto ResolverValueDec;

auto TwosComp;

auto RealVoltage;

auto SetValue;

auto rc;

auto i;

auto Resolvers_Row;

auto rate;

auto Angle;

auto bits[];

auto bit[];

auto twos[];

auto Units;

auto Port;

auto Resolvers_RowCount $=20$;

auto MaxVoltage = 9.0;

auto Tolerance;

auto ResRef;

auto Function;

Resolvers = "EMI Air_Data resolvers.xls";

$\mathrm{rc}=$ ddt_open (Resolvers, DDT_MODE_READ);

if $\left(\mathrm{rc} !=\mathrm{E} \_\mathrm{OK} \& \& \mathrm{rc} !=\mathrm{E} \_\right.$FILE_OPEN)

pause("Cannot open table.");

for $($ Resolvers_Row $=1$; Resolvers_Row $<=20$; Resolvers_Row ++ )

\{

ddt_set_row(Resolvers,Resolvers_Row);

Label = ddt_val(Resolvers, "Label")

Designation = ddt_val(Resolvers, "Designation");

ExpectedValue $=$ ddt_val(Resolvers, "Expected_Value_Dec");

Units = ddt_val(Resolvers, "Units");

Angle $=$ ddt_val(Resolvers, "Angle"); 
Port $=$ ddt_val(Resolvers, "Port");

ResolverValue = "";

TwosComp = "";

Function = "Air_Data";

for $(i=1 ; i<=10 ; i++)$

set_window ("Air_Data HW RX", 5);

obj_wait_info("SysListView32","displayed",1,10);

list_activate_item ("SysListView32", Port ";" Label);

if(win_exists("Label Data...", 1)!=E_OK)

list_activate_item ("SysListView32", Port ";" Label)

set_window("Label Data...", 1);

obj_get_text("Rate", rate);

obj_get_text("Value", RealValue);

win_close ("Label Data...");

if (rate $<1 \& \& \mathrm{i}<10)$ \#Try again due to Update Delay in ACO continue;

if (rate $<1 \& \& \mathrm{i}==10)$ \#Label is not transmitted

tl_step("Check " Function " Label " Label, FAIL, Function " Label " Label " Could not be checked, it was not transmitted.", Function " Label " Label " should be transmitted" , Function " Label " Label " Was not transmitted");

\# Check Resolver Values

if (rate $>=1)$

\{

RealValue = repl_str(RealValue," ","');

ResolverValue = substr(RealValue, 7,16$)$;

\# Need Twos Compliment if MSB is

if $(\operatorname{substr}($ ResolverValue, 1,1$)==1)$

for $(i=2 ; i<=16 ; i++)$

\{

$$
\text { twos[i] = substr(ResolverValue, i, 1); }
$$




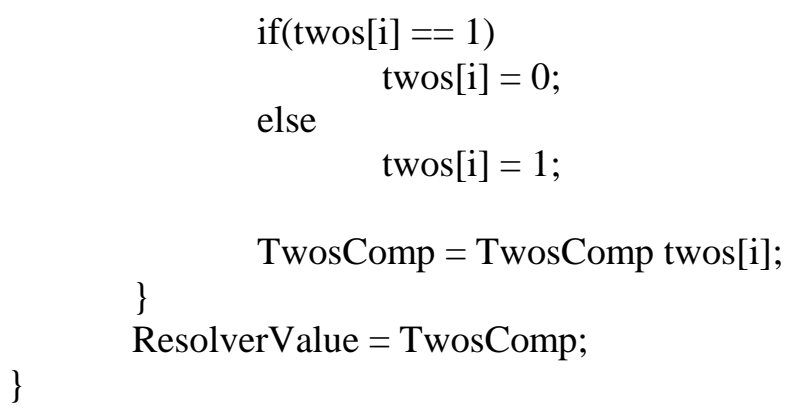

\# Now Convert Binary to Decimal and Multiply by $305 \mathrm{uV}$

ResolverValueDec $=0$;

for (i=0; i<length(ResolverValue); i++)

\{

bit[i] = substr $($ ResolverValue, length $($ ResolverValue $)-i, 1)$;

\}

ResolverValueDec $+=\operatorname{bit}[\mathrm{i}] * 2^{\wedge} \mathrm{i}$;

RealVoltage $=$ ResolverValueDec $* .000305 ;$

if $(\operatorname{substr}($ RealValue, 7,1$)==1)$

RealVoltage $=($ ResolverValueDec $*-0.000305)-.000305$;

if (Resolvers_Row $<=8$ )

\{

if (ExpectedValue-MaxVoltage*. $1<$ RealVoltage \&\& RealVoltage <

ExpectedValue+MaxVoltage*.1)

tl_step("Check " Designation, PASS, Designation " was set to within 10\% Tolerance at Angle " Angle "on Air_Data ODB \#" Port ". Actual voltage: " RealVoltage " " Units ". Expected Voltage: "ExpectedValue " " Units, "Expected Voltage: " ExpectedValue " " Units, "Actual Voltage: " RealVoltage " " Units);

else

tl_step("Check " Designation, FAIL, Designation " was NOT within 10\% Tolerance at Angle " Angle "on Air_Data ODB \#" Port ". Actual voltage: " RealVoltage " " Units ". Expected Voltage: "ExpectedValue " " Units, "Expected Voltage: " ExpectedValue " " Units, "Actual Voltage: " RealVoltage " " Units); 
if (Resolvers_Row $>8$ \&\& Resolvers_Row $<=18)$

\{

if (ExpectedValue-MaxVoltage*. $1<$ RealVoltage \&\& RealVoltage <

ExpectedValue+MaxVoltage*.1)

tl_step("Check " Designation, PASS, Designation " was set to within $10 \%$ Tolerance on Air_Data ODB \#" Port ". Actual value: " RealVoltage " " Units ". Expected value:

"ExpectedValue " " Units, "Expected value: " ExpectedValue " " Units, "Actual value: " RealVoltage " " Units);

else

tl_step("Check " Designation, FAIL, Designation " was NOT within 10\% Tolerance on Air_Data ODB \#" Port ". Actual value: " RealVoltage " " Units ". Expected value:

"ExpectedValue " " Units, "Expected value: " ExpectedValue " " Units, "Actual value: " RealVoltage " " Units);

\}

if (Resolvers_Row $>18 \& \&$ Resolvers_Row $<=20)$

\{

RealVoltage $=$ ResolverValueDec $/ .000305$

if (ExpectedValue*.9 < RealVoltage \&\& RealVoltage $<$ ExpectedValue*1.1)

tl_step("Check " Designation, PASS, Designation " was set to within $10 \%$ Tolerance on Air_Data ODB \#" Port ". Actual value: " RealVoltage " " Units ". Expected value:

"ExpectedValue " " Units, "Expected value: " ExpectedValue " " Units, "Actual value: " RealVoltage " " Units);

else

tl_step("Check " Designation, FAIL, Designation " was NOT within 10\% Tolerance on Air_Data ODB \#" Port ". Actual value: " RealVoltage " " Units ". Expected value: "ExpectedValue " " Units, "Expected value: " ExpectedValue " " Units, "Actual value: " RealVoltage " " Units);

break;

report_msg("End of Air_Data Resolver Test Function");

ddt_close(Resolvers); 


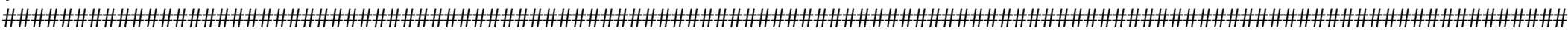

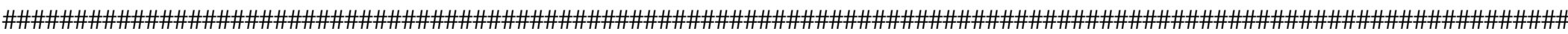
public function EMI_Check_Output_Discretes()

$$
\{
$$

auto ODSC;

auto rc;

auto DDT_MODE_READ;

auto ODSC_Row;

auto Label;

auto Discrete;

auto value;

auto Output;

auto Mask;

auto Ground;

auto Open;

auto i;

auto Designation;

auto ODSC_RowCount $=6$;

auto Batt;

auto DC;

auto Navigation;

auto Align;

auto Function;

auto Expected;

auto Port;

auto RealValue;

auto array[];

auto Bit[];

auto Setting;

auto rate;

ODSC = "EMI output discretes.xls";

rc $=$ ddt_open (ODSC, DDT_MODE_READ);

if (rc!= E_OK \& \& rc != E_FILE_OPEN)

pause("Cannot open table.");

\#Check Output Discretes with Input Enabled 
for(ODSC_Row $=1$; ODSC_Row <= ODSC_RowCount; ODSC_Row ++)

\{

ddt_set_row(ODSC,ODSC_Row);

Discrete = ddt_val(ODSC, "Input");

Output $=$ ddt_val(ODSC, "Output");

Designation = ddt_val(ODSC, "Designation");

Function = ddt_val(ODSC, "Function");

Label = ddt_val(ODSC, "Label");

Expected = ddt_val(ODSC, "Expected_Bit");

Port $=$ ddt_val(ODSC, "Port");

Setting $=\overline{d d t} \_$val $($ODSC, "Setting");

for $(i=1 ; i<=3 ; i++)$

\{

set_window ("EMI " Function " RX", 5);

obj_wait_info("SysListView32","displayed",1,10);

list_activate_item ("SysListView32", Port ";" Label);

if(win_exists("Label Data...", 1)!=E_OK)

list_activate_item ("SysListView32", Port ";" Label);

set_window("Label Data...", 1);

obj_get_text("Rate", rate);

obj_get_text("Value", RealValue);

win_close ("Label Data...");

if (rate $<1 \& \& \mathrm{i}<3$ ) \#Try one more time due to Update Delay in ACO continue;

if (rate $<1 \& \& \mathrm{i}==3$ ) \#Label is not transmitted

tl_step("Check " Function " Label " Label, FAIL, Function " Label " Label " Could not be checked, it was not transmitted.", Function " Label " Label " should be transmitted" , Function " Label " Label " Was not transmitted");

if $($ rate $>=1)$

\{

RealValue = repl_str(RealValue," ","');

\# Convert ACO Bits String to Actual Bits set 


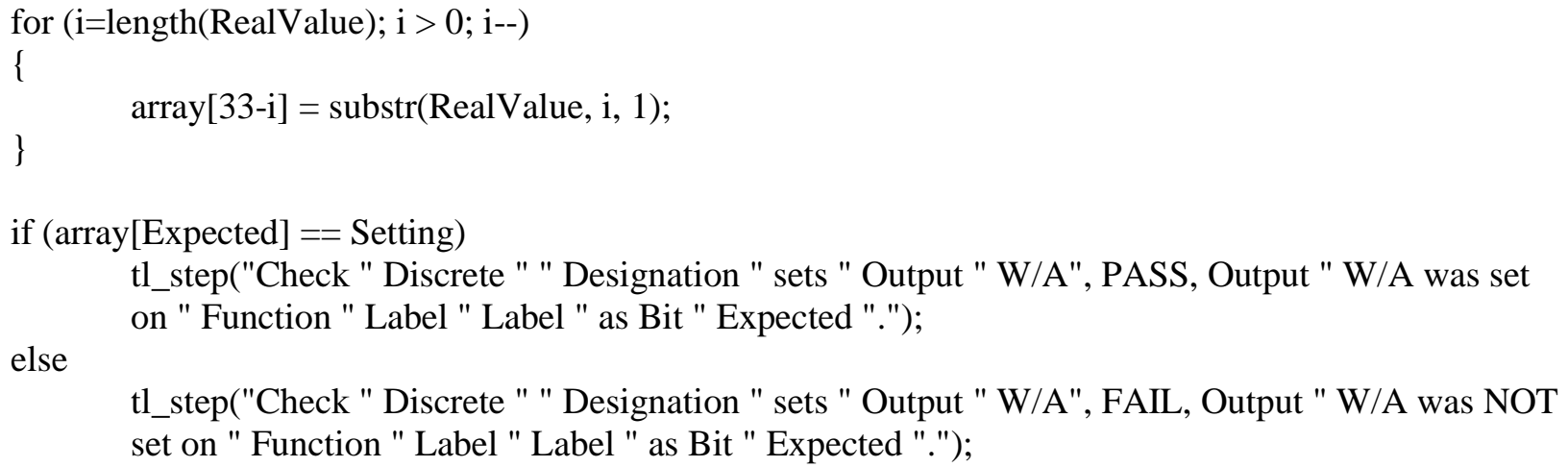


auto ReturnValue;

auto MessageCount1;

auto MessageCount2;

auto RealValueMSH;

auto RealValueLSH;

auto MessageCount[];

auto ECC_ErrorCount;

auto ECC [];

auto bit[];

auto SA_TableRow;

auto CountValue;

auto Message[];

auto ErrorMessageCount;

auto Error[];

auto ErrorCount;

SA = "EMI sensor assembly data.xls";

rc = ddt_open(SA, DDT_MODE_READ);

if ( $\mathrm{rc} !=$ E_OK \& \& rc != E_FILE_OPEN)

pause("Cannot open table.");

\# Check Processor Control Registers for Errors

for $($ SA_TableRow $=1$; SA_TableRow $<1$; SA_TableRow++)

\{

ddt_set_row(SA, SA_TableRow);

Label = ddt_val(SA, "Label");

Description = ddt_val(SA, "Description");

Port $=$ ddt_val(SA, "Port");

Expected = ddt_val(SA, "Expected");

Mask $=$ ddt_val(SA, "Mask");

for $(\mathrm{i}=1 ; \mathrm{i}<=3 ; \mathrm{i}++)$

\{

set_window ("EMI Air_Data RX", 5);

obj_wait_info("SysListView32","displayed",1,10);

list_activate_item ("SysListView32", Port ";" Label);

if(win_exists("Label Data...", 1)!= E_OK) 
list_activate_item ("SysListView32", Port ";" Label);

set_window("Label Data...", 1);

obj_get_text("Rate", rate);

obj_get_text("Value", RealValue);

win_close ("Label Data...");

if (rate $<1 \& \& \mathrm{i}<3)$ \#Try one more time due to Update Delay in ACO continue;

if (rate $<1 \& \& \mathrm{i}==3$ ) \#Label is not transmitted

tl_step("Check Air_Data Label " Label, FAIL, "Air_Data Label " Label " Could not be checked, it was not transmitted.", "Air_Data Label " Label " should be transmitted" , "Air_Data Label " Label " Was not transmitted");

if (rate $>=1)$

\{

RealValue = repl_str(RealValue," ","");

if $($ Label $==151)$

\{

CountValue $=$ substr $($ RealValue $, 15,8)$;

\# Now Convert Binary to Decimal (Error Count)

for $(\mathrm{i}=0$; $i<$ length(CountValue); $\mathrm{i}++$ )

\{

ECC $[i]=\operatorname{substr}($ CountValue, length(CountValue $)-i, 1)$;

\}

ECC_ErrorCount $+=\mathrm{ECC}[\mathrm{i}] * 2^{\wedge} \mathrm{i}$;

if (ECC_ErrorCount > 0)

tl_step("Check TESCR1 Single ECC Error Count", FAIL, ECC_ErrorCount " Single

ECC Error(s) Present on Air_Data Label " Label, "No ECC Error(s) Present.",

ECC_ErrorCount " Single ECC Error(s) Present.");

else

tl_step("Check TESCR1 Single ECC Error Count", PASS, "No ECC Error(s) Present.", "No ECC Error(s) Present on Air_Data Label " Label, "No Single ECC Error(s)

Present."); 
\# Convert ACO Bits String to Actual Bits set

for ( $\mathrm{i}=0$; $\mathrm{i}<$ length(RealValue); $\mathrm{i}++$ )

\{

$\operatorname{array}[\mathrm{i}+1]=\operatorname{substr}($ RealValue $, \mathrm{i}+1,1)$;

if $(\operatorname{array}[\mathrm{i}+1]==1)$

array $[i+1]=32-\mathrm{i} ", "$

else $\operatorname{array}[\mathrm{i}+1]=$ " ";

\}

switch(Label)

\{

case 150 :

ActualBits = "Bit(s) " array[13] array[12] array[11] array[10] array[9] array[8];

break;

case 152:

ActualBits = "Bit(s) " array[22] array[21] array[20] array[19] array[18] array[17]

array[16] array[15] array[9] array[8];

break;

case 153:

ActualBits = "Bit(s) " array[18] array[17] array[16] array[15] array[14] array[13] array[12] array[11] array[10] array [9] array[8] array[7];

break;

WRU_MaskAndCompareBinString(Expected, RealValue, Mask, ReturnValue);

if $($ ReturnValue $==1)$

tl_step("Check " Description, PASS, "No " Description " Found on Label " Label, "No "

Description " Found.", "No " Description " Found."); 
tl_step("Check " Description, FAIL, Description " Found on Label " Label ". Check Error Corresponding to " ActualBits " of Label " Label ".", "No " Description " Found.", "Error Corresponding to " ActualBits " of Label " Label ".");

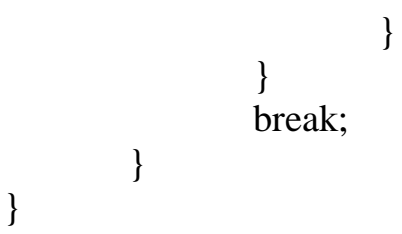

\section{\# Check First Label Value}

ddt_set_row(SA, SA_TableRow);

Label = ddt_val(SA, "Label");

Description = ddt_val(SA, "Description");

Port = ddt_val(SA, "Port");

Expected = ddt_val(SA, "Expected");

Mask = ddt_val(SA, "Mask");

if(Label==154 || Label==156 || Label==164 || Label==170)

\{

for $(\mathrm{n}=1 ; \mathrm{n}<=2 ; \mathrm{n}++)$

\{

\# Check First Label Value

ddt_set_row(SA, SA_TableRow);

Label = ddt_val(SA, "Label");

Description = ddt_val(SA, "Description");

Port $=$ ddt_val(SA, "Port");

Expected = ddt_val(SA, "Expected");

Mask = ddt_val(SA, "Mask");

for $(i=1 ; i<=3 ; i++)$

\{

set_window ("EMI Air_Data RX", 5);

obj_wait_info("SysListView32","displayed",1,10); 
list_activate_item ("SysListView32", Port ";" Label);

if(win_exists("Label Data...", 1)!= E_OK)

list_activate_item ("SysListView32", Port ";" Label);

set_window("Label Data...", 1);

obj_get_text("Rate", rate);

obj_get_text("Value", RealValue);

win_close ("Label Data...");

if (rate $<1 \& \& \mathrm{i}<3$ ) \#Try one more time due to Update Delay in ACO continue;

if (rate $<1 \& \& \mathrm{i}==3$ ) \#Label is not transmitted

tl_step("Check Air_Data Label " Label, FAIL, "Air_Data Label " Label " Could not be checked, it was not transmitted.", "Air_Data Label " Label " should be transmitted" ,

"Air_Data Label " Label " Was not transmitted");

if $($ rate $>=1)$

\{

RealValue = repl_str(RealValue, " ", "");

RealValueMSH = substr(RealValue, 7,16$)$;

\#Check Next Label Value

SA_TableRow $=$ SA_TableRow + ;

ddt_set_row(SA, SA_TableRow);

Label = ddt_val(SA, "Label");

Description = ddt_val(SA, "Description");

Port $=$ ddt_val(SA, "Port");

Expected = ddt_val(SA, "Expected");

Mask $=$ ddt_val(SA, "Mask");

for $(i=1 ; i<=3 ; i++)$

\{

set_window ("EMI Air_Data RX", 5);

obj_wait_info("SysListView32","displayed",1,10);

list_activate_item ("SysListView32", Port ";" Label); 
if(win_exists("Label Data...", 1)!= E_OK)

list_activate_item ("SysListView32", Port ";" Label);

set_window("Label Data...", 1);

obj_get_text("Rate", rate);

obj_get_text("Value", RealValue);

win_close ("Label Data...");

if (rate $<1 \& \& \mathrm{i}<3$ ) \#Try one more time due to Update Delay in ACO continue;

if (rate $<1 \& \& \mathrm{i}==3$ ) \#Label is not transmitted

tl_step("Check Air_Data Label " Label, FAIL, "Air_Data Label " Label " Could not be checked, it was not transmitted.", "Air_Data Label " Label

" should be transmitted" , "Air_Data Label " Label " Was not transmitted");

if $($ rate $>=1)$

\{

RealValue = repl_str(RealValue, " ", "");

RealValueLSH = substr(RealValue, 7, 16);

Count $=$ RealValueMSH RealValueLSH;

MessageCount $[\mathrm{n}]=0$;

\# Now Convert Binary to Decimal

for (i=0; i<length(Count); i++)

Message $[\mathrm{i}]=$ substr( Count, length $($ Count $)-\mathrm{i}, 1)$;

MessageCount $[\mathrm{n}]+=$ Message $[\mathrm{i}] * 2^{\wedge} \mathrm{i}$;

\}

if $(n==1)$

SA_TableRow $=$ SA_TableRow-1;

\}

break; 
else

\{

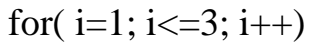

if (rate $<1 \& \& \mathrm{i}<3$ ) \#Try one more time due to Update Delay in ACO continue;

if (rate $<1 \& \& \mathrm{i}==3$ ) \#Label is not transmitted

tl_step("Check Air_Data Label " Label, FAIL, "Air_Data Label " Label " Could not be checked, it was not transmitted.", "Air_Data Label " Label " should be transmitted" , "Air_Data Label " Label " Was not transmitted");

if $($ rate $>=1)$

\{

RealValue = repl_str(RealValue, " ", "");

RealValueMSH = substr(RealValue, 7, 16);

\#Check Next Label Value

SA_TableRow $=$ SA_TableRow + ;

ddt_set_row(SA, SA_TableRow);

Label = ddt_val(SA, "Label"); 
Description $=$ ddt_val(SA, "Description");

Port $=$ ddt_val(SA, "Port");

Expected $=$ ddt_val(SA, "Expected");

Mask $=$ ddt_val(SA, "Mask");

\section{for $(\mathrm{i}=1 ; \mathrm{i}<=3 ; \mathrm{i}++)$}

\{

set_window ("EMI Air_Data RX", 5);

obj_wait_info("SysListView32","displayed",1,10);

list_activate_item ("SysListView32", Port ";" Label);

if(win_exists("Label Data...", 1)!= E_OK)

list_activate_item ("SysListView32", Port ";" Label);

set_window("Label Data...", 1);

obj_get_text("Rate", rate);

obj_get_text("Value", RealValue);

win_close ("Label Data...");

if (rate $<1 \& \& \mathrm{i}<3$ ) \#Try one more time due to Update Delay in ACO continue;

if (rate $<1 \& \& \mathrm{i}==3$ ) \#Label is not transmitted

tl_step("Check Air_Data Label " Label, FAIL, "Air_Data Label " Label " Could not be checked, it was not transmitted.", "Air_Data Label " Label " should be transmitted" , "Air_Data Label " Label " Was not transmitted");

if $($ rate $>=1)$

\{

RealValue = repl_str(RealValue, " ", "");

RealValueLSH = substr(RealValue, 7, 16);

ErrorCount $=$ RealValueMSH RealValueLSH;

ErrorMessageCount $=0$;

\# Now Convert Binary to Decimal

for (i=0; i<length(ErrorCount); i++) 


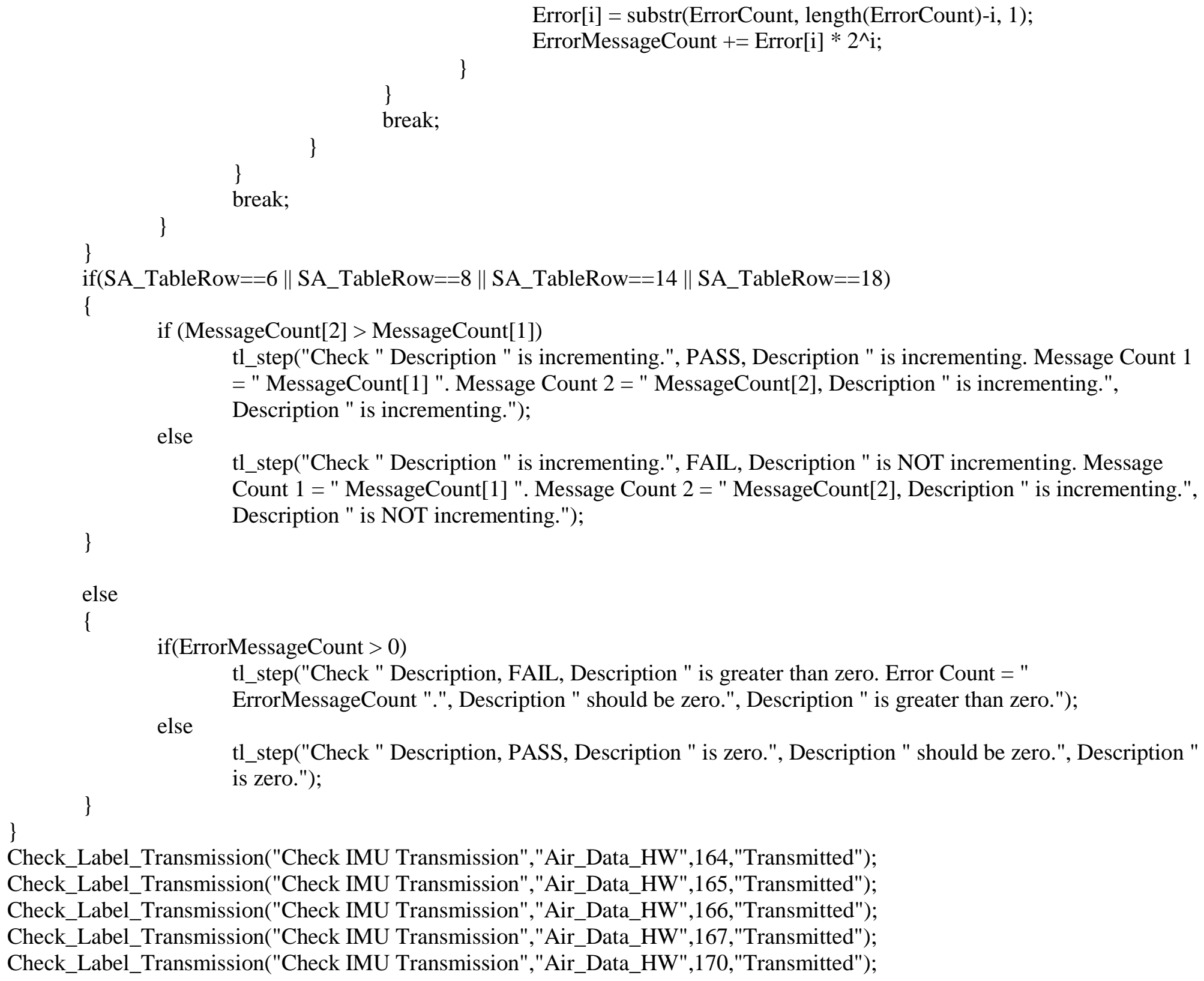


Check_Label_Transmission("Check IMU Transmission","Air_Data_HW",171,"Transmitted");

Check_Label_Transmission("Check IMU Transmission","Air_Data_HW",172,"Transmitted");

Check_Label_Transmission("Check IMU Transmission","Air_Data_HW",173,"Transmitted");

report_msg("End of AFDX/SA Message and Error Count Check.");

ddt_close(SA);

\}

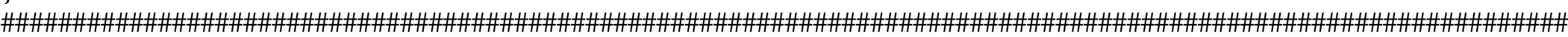

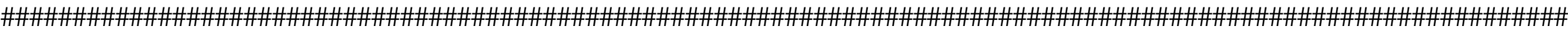
public function EMI_Recorded_Labels(Label_Table)

auto Record;

auto array[];

auto Label;

auto Label_Row;

auto rc;

auto i;

auto Expected;

auto Label_Number;

auto Data;

auto Event;

auto Function;

split(Label_Table, array, " ");

Function $=$ array $[1]$;

if (Function $==$ "Air_Data")

Label = "Air_Data Expected Data.xls";

else

Label = "GPS_Navigation Expected Data.xls";

rc $=$ ddt_open(Label, DDT_MODE_READ);

if $\left(\mathrm{rc} !=\mathrm{E} \_\mathrm{OK} \& \& \mathrm{rc} !=\mathrm{E} \_\right.$FILE_OPEN)

pause("Cannot open expected data table.");

Record = "' Label_Table ".xls";

rc = ddt_open(Record, DDT_MODE_READ);

if (rc!= E_OK \& \& rc != E_FILE_OPEN)

pause("Cannot open record data table.")

for $($ Label_Row = 1; Label_Row < 500; Label_Row++)

\{ 


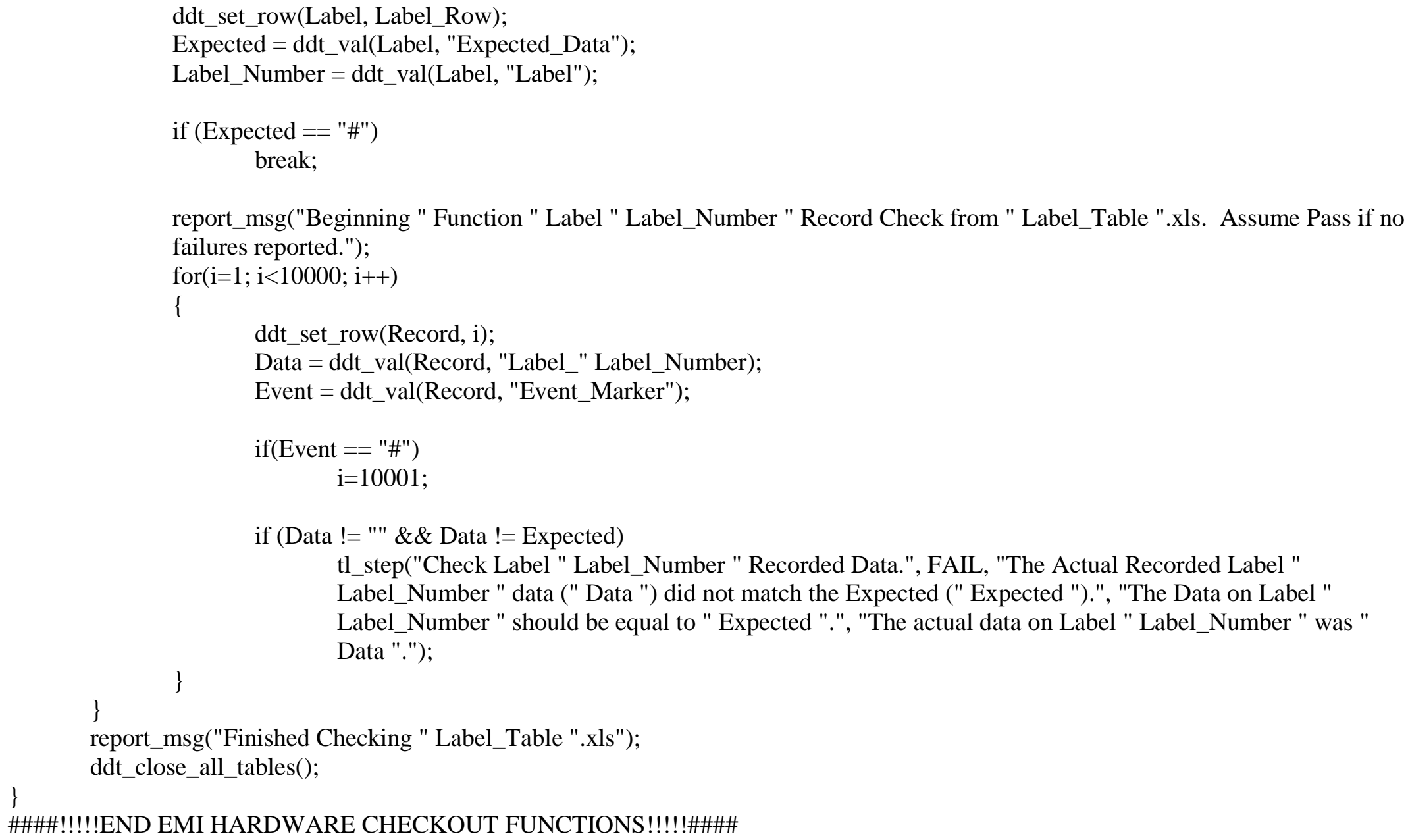

